\title{
Programação de produção e dimensionamento de lotes para flowshop
}

Márcio Antônio Ferreira Belo Filho 

SERVIÇO DE PÓS-GRADUAÇÃO DO ICMC-USP

Data de Depósito: 08/10/2010

Assinatura:

\title{
Programação de produção e dimensionamento de lotes para flowshop
}

\author{
Márcio Antônio Ferreira Belo Filho
}

Orientadora: Profa. Dra. Maristela Oliveira dos Santos

Dissertação apresentada ao Instituto de Ciências Matemáticas e de Computação - ICMC-USP, como parte dos requisitos para obtenção do título de Mestre em Ciências - Ciências de Computação e Matemática Computacional.

USP - São Carlos

Outubro/2010 




\section{Resumo}

O problema integrado de programação de produção e dimensionamento de lotes em ambiente flowshop consiste em estabelecer tamanhos de lotes de produção e alocar máquinas para processá-los dentro de um horizonte de planejamento, em uma linha de produção com máquinas dispostas em série. O problema considera que a demanda deve ser atendida sem atrasos, que a capacidade das máquinas deve ser respeitada e que as preparações de máquinas são dependentes da sequência de produção e preservadas entre períodos do horizonte de planejamento. O objetivo é determinar uma programação de produção visando minimizar os custos de preparação de máquina, de produção e de estoque. Um modelo matemático da literatura é apresentado assim como procedimentos para obtenção de limitantes inferiores. Além disso, abordamos o problema por meio de distintas versões da metaheurística Times Assíncronos (A-Teams). Os procedimentos propostos foram comparados com heurísticas da literatura baseadas em Programação Inteira Mista $(M I P)$. As metodologias desenvolvidas e os resultados obtidos são apresentados nesta dissertação.

Palavras-chave: flowshop, dimensionamento de lotes, programação da produção, times assíncronos. 



\section{Abstract}

The integrated production scheduling and lot sizing problem in a flowshop environment consists in establishing production lot sizes and alocate machines to process them inside a planning horizon, in a production line with machines arranged in series. The problem considers that demand must be met without backlogging, the capacity of the machines must be respected, machine setup are sequence-dependent and preserved between periods of the planning horizon. The objective is to determine a production schedule to minimize the setup, production and inventory costs. A mathematical model from the literature is presented as well as procedures for obtaining lower bounds. In addition, we propose to address the problem through different versions of the metaheuristic Asynchronous Teams (A-Teams). The procedures were compared with literature heuristics based on Mixed Integer Programming $(M I P)$. The developed methodologies and the obtained results are presented in this dissertation.

Keywords: flowshop, lot sizing, production scheduling, asynchronous teams. 



\section{Agradecimentos}

A Deus primeiramente, por ter me guiado através dos problemas de otimização da minha vida. Venho a muito tempo trilhando por pontos extremos, regiões factíveis e infactíveis e Ele, como grande otimizador que é, sempre me empresta alguns problemas que consigo suportar.

À minha família que sempre me deu carinho e suporte. Graças a ela aprendi virtudes importantes, como ter honra, expressar humildade, ser paciente e terno e acima de tudo, ser amigo.

À minha grande família, em especial meu avô Gerolino e minhas avós Maria Amélia e Anita, vocês são fontes de ternura e sempre lembro de vocês com lágrimas nos olhos. A todos os meus tios, primos, parentes distantes. Agradeço muito a minha prima, quase irmã, a Hérica. Você é uma das pessoas que mais me faz sentir bem.

À minha namorada, a Van, por tudo que trilhamos neste tempo que estamos juntos. A sua amizade, compreensão, alegria e beleza me fascinam.

À minha orientadora, professora doutora Maristela Oliveira dos Santos, que me iniciou nos estudos em otimização e me guiou por iniciações científicas e mestrado. A ela sou muito grato por ter me apresentado a área que me identifico e pretendo trabalhar futuramente.

Ao meu coorientador, o professor doutor Cláudio Nogueira de Meneses, que por meio de incontáveis conselhos, conversas fraternas e ensinamentos me proporcionou um grande e rico aprendizado.

Ao conjunto de professores que pacientemente me ensinaram diversos conhecimentos começando pela minha infância até aqueles professores que pacientemente me ensinarão no futuro. Espero poder em breve repassar esta sabedoria que a mim foi confiada tão bem quanto vocês me passaram.

Ao Laboratório de Otimização (LOT), por disponibilizar conhecimento, amizades e inspiração. Momentos passados no laboratório juntamente com as pessoas que o coabitam me fazem sempre querer estar neste local de trabalho. Dentre os companheiros do LOT, vale ressaltar alguns: 
Ao Victor pelos conselhos amigos, pelos ensinamentos no laboratório, pelas corridas no kartodromo, pelos diversos espetinhos e cervejas que muitas vezes procuramos em São Carlos, pelas festas que fomos/fizemos (inclusive as chapas), pelas esfihas de espinafre do habib's, pelas viagens etc. Enfim, um grande amigo, com um grande futuro.

À Tamara por sempre fazer todos a sorrir, por dar conselhos amigos a qualquer hora, por me acompanhar sempre num chopp, pelas festas que fomos/fizemos, pelas viagens compartilhadas etc. Outra grande amiga, com um carisma imenso e um sorriso cordial.

À Marina, por tantas conversas, desde que éramos simples graduandos, quando fingia que dava monitoria de métodos numéricos. Adoro sempre conversar e ouvir os seus conselhos.

Aos companheiros Marcos Furlan, Aline (credo!), Douglas, Pedro, Mariá, Mayron, Felipe, Rafael, Scheila, Cláudinha, Pâmela, Ana Paula, Gabriela, enfim, todos do LOT.

Aos professores do grupo de otimização e pertencentes ao LOT, Marcos Arenales, Franklina Toledo, Alysson Costa, Marina Andretta e o recente professor Elias. Espero ter muitos conhecimentos a compartilhar com estas pessoas após ter aprendido tanto.

Àqueles que estavam presentes na minha qualificação, especialmente banca, formadas pelos professores Reinaldo Morabito e Franklina Toledo. Suas sugestões foram essenciais para o meu trabalho.

À presente banca de mestrado, cujas sugestões, conselhos e correções serão com certeza essenciais e engrandecerão o meu trabalho.

À minha república que hoje é a minha atual família de São Carlos, por todos os seus moradores, desde aqueles que a formaram (Bruno Max, Dário, Alex, Rodrigo e eu), todos que já sairam (Márcio André, Giliarde, Albert, Hinnerk, Florian, Santiago, Juari) e todos aqueles que estão nela hoje (Brahma, Marcelão, Saulo, Maurício, Gabriel, Mehdi, Bixo). A todos que passaram por ela, um dia, uma semana, um mês ou mais. Carrego comigo toda a fraternidade e alegria contagiante que vocês representam.

Aos meus amigos de São Carlos, desde a época que comecei, como bixo em engenharia mecatrônica, aqueles que cursaram comigo matemática aplicada e aqueles que cursam comigo o doutorado. Toda a turma formada pelos Goianos.

A todos meus amigos que deixei em Goiânia quando parti para estudar aqui em São Carlos. Alguns laços se romperam, outros estão mais fortes. Em especial, Brunno Mendes, Sir Fabiano, Morenno (sim, esse é o nome dele), Maýra Tolentino, Rosalinda, 
Verena, Gabriel, Flávio César, dentre outros tantos.

A todas pessoas que me deram uma estrutura para poder realizar a minha pesquisa. Todas as agências de fomento, em especial a CNPq que fornece a minha bolsa, todos aqueles que trabalham na instituição USP, desde professores capacitados, funcionários que mantêm um bom ambiente de estudos e trabalho e os alunos.

Agradeço por último a todos aqueles que não foram citados. Agradeço muito a todos que participaram de minha vida. Vocês contribuiram na minha formação e na minha vida, por isso sou muito grato a vocês. 



\section{Sumário}

Lista de Figuras

p. 1

1 Introdução

p. 3

2 Revisão da Literatura

3 Definição e Modelagem do Problema

3.1 Modelo Matemático para o Caso Permutacional . . . . . . . . . . p. 20

3.2 Modelo Matemático Reduzido . . . . . . . . . . . . . . . . . p. 22

3.3 Exemplo Numérico . . . . . . . . . . . . . . . . . . . . . p. 24

4 Procedimentos de Solução da Literatura p. 29

4.1 Limitantes Inferiores $\ldots \ldots \ldots \ldots \ldots$. . . . . . . . . . . . . . . . 29

4.2 Heurísticas baseadas em Horizonte Rolante . . . . . . . . . . . . . p. 31

4.2.1 Primeira Heurística de Horizonte Rolante $($ RH1 $)$. . . . . . . . . p. 31

4.2.2 Segunda Heurística de Horizonte Rolante $($ RH2) . . . . . . . . . . p. 32

4.2.3 Terceira Heurística de Horizonte Rolante $($ RH3 $) \ldots . . .$. p. 32

4.3 Heurísticas Relax-and-Fix . . . . . . . . . . . . . p. 33

4.3.1 Heurística Relax-and-Fix $(R F) \ldots \ldots$. . . . . . . . 34

5 Procedimentos de Solução Propostos p. 35

5.1 Times Assíncronos . . . . . . . . . . . . . . . . p. 35

5.2 Times Assíncronos Proposto $(A T) \ldots \ldots \ldots \ldots$. . . . . . . . 36

5.2.1 Estrutura de Dados e Formato de Representação . . . . . . . . . . p. 37 
5.2 .2 Agentes Construtores . . . . . . . . . . . . . . . . p. 39

5.2 .3 Agentes Destruidores . . . . . . . . . . . . . . . p. 41

5.2.4 Agentes de Melhoria . . . . . . . . . . . . . . . p. 41

5.3 Times Assíncronos Híbrido $(A T H) \ldots \ldots \ldots$. . . . . . . . 46

5.3.1 Agentes Construtores . . . . . . . . . . . . . p. 47

$5.3 .2 \quad$ Agentes Destruidores . . . . . . . . . . . . . . . . p. 49

5.3 .3 Agentes de Melhoria . . . . . . . . . . . . . . p. 49

6 Experimentos Computacionais p.51

6.1 Ambiente de Teste . . . . . . . . . . . . . . . . p. 51

6.2 Problemas-Teste Gerados . . . . . . . . . . . . . . . . . . p. 51

6.3 Parâmetros dos Procedimentos . . . . . . . . . . . . . . . p. 54

6.4 Resultados Computacionais . . . . . . . . . . . . . . p. 54

6.4.1 Procedimentos da Literatura . . . . . . . . . . . . . p. 55

6.4.2 Metaheurísticas Propostas . . . . . . . . . . . . p. 59

6.4 .3 Resultados Finais . . . . . . . . . . . . . . . . p. 62

7 Conclusões e Perspectivas p. 65

$\begin{array}{lr}\text { Referências Bibliográficas } & \text { p. } 67\end{array}$

$\begin{array}{ll}\text { Apêndice A - Gráficos de Gantt } & \text { p.73 }\end{array}$

Apêndice B - Perfil de Desempenho de Dolan e Moré (2002) p.75

$\begin{array}{ll}\text { Apêndice C - Resultados Gerais } & \text { p.77 }\end{array}$

$\begin{array}{lr}\text { Apêndice D - Tempos Computacionais } & \text { p. } 87\end{array}$ 


\section{Lista de Figuras}

1 Diagrama de Gantt representando interação vertical. . . . . . . . . . . . p. 20

2 Diagrama de Gantt da primeira solução. . . . . . . . . . . . . . . . . p. 26

3 Diagrama de Gantt da segunda solução. . . . . . . . . . . . . . . . p. 26

4 Estratégia de horizonte rolante baseada em Mohammadi et al. (2010a). . p. 32

5 Exemplo de Arquitetura A-Teams. . . . . . . . . . . . . p. 36

6 Estrutura de Dados do Problema. . . . . . . . . . . . . . . p. 38

7 Representação Compacta de uma Solução para o Problema $M M P$. . . . . p. 38

8 Representação da solução gerada pelo agente construtor $1 A g C 1$. . . . . p. 40

9 Representação da solução gerada pelo agente construtor 2 (AgC2). . . . . p. 41

10 Vizinhanças dos dois primeiros agentes de melhoria. . . . . . . . . . . p. 42

11 Todas as soluções geradas pelo agente de melhoria $3(A g B I) \ldots$. . . . . . p. 43

12 Solução gerada pelo agente de melhoria $4(A g C O) . \quad \ldots \ldots$. . . . . . . p. 43

13 Solução gerada pelo agente de melhoria $5(A g A P) \ldots \ldots$. . . . . . . . p. 44

14 Solução gerada pelo agente de melhoria $6(A g E P) \ldots \ldots$. . . . . . . p. 45

15 Solução gerada pelo agente de melhoria $9(A g A E P) \ldots \ldots$. . . . . . . . p. 46

16 Diagrama de Gantt apresentando a capacidade mínima. . . . . . . . . . . p. 52

17 Perfil de Desempenho dos procedimentos avaliados. . . . . . . . . . p. 58

18 Perfil de Desempenho das metaheurísticas times assíncronos. . . . . . . p. 61

19 Perfil de Desempenho das metaheurísticas times assíncronos híbridas. . . p.61

20 Perfil de Desempenho dos procedimentos avaliados. . . . . . . . . . p. 63

21 Rosto de Henry Gantt. . . . . . . . . . . . . . . . . . . . . . p. 73

22 Gráfico de Gantt representando uma linha de produção sem blocking. p.74 
23 Gráfico de Gantt representando uma linha de produção com blocking. . . p.74 


\section{Introdução}

As indústrias buscam melhorar o planejamento de produção para obter um melhor posicionamento na concorrência dos mercados, visando minimizar os custos produtivos e aumentar a eficiência de sua cadeia produtiva. De acordo com Pochet e Wolsey (2006) o planejamento de produção (Production Planning) é o planejamento de aquisição de recursos e matérias-primas, assim como o planejamento das atividades relacionadas à transformação da matéria-prima em produtos satisfazendo à demanda de consumidores da maneira mais eficiente e econômica possível. O planejamento de produção envolve decisões de dimensionamento de lotes (lot sizing), sequenciamento (sequencing) e programação da produção (production scheduling) (POCHET; WOLSEY, 2006). Assim os problemas de planejamento da produção abordam decisões de curto e médio prazo, ou seja, decisões dos níveis tático e operacional. Os problemas de programação da produção consistem na alocação de recursos a tarefas determinando seus instantes de início e término, num período de tempo. O problema de sequenciamento de lotes consiste em estabelecer sequências dos lotes de produtos a serem produzidos. O problema de dimensionamento de lotes consiste em definir os tamanhos dos lotes de produção a serem produzidos ao

longo do tempo, para que uma determinada demanda seja atendida. Diversos trabalhos na literatura tratam de cada problema separadamente.

O problema de programação da produção é dependente do ambiente de produção, já que os tempos de cada unidade de um determinado lote dependem da sua rota por meio dos estágios de produção, da disponibilidade desses estágios e dos tempos de outras unidades do mesmo lote ou mesmo de outros produtos. Existem vários tipos de linhas de produção definidos de acordo com a configuração das máquinas e das rotas dos produtos nas máquinas. Em Pinedo (2008) os ambientes de produção são classificados como: única máquina, máquinas paralelas, flowshop, jobshop e openshop, além das variações de máquinas paralelas que podem ser idênticas, uniformes e não-relacionadas, e de flowshop híbrido, jobshop híbrido e openshop híbrido, que envolvem estágios de máquinas paralelas. O ambiente de produção flowshop é caracterizado por lotes de produtos sendo produzidos 
por uma quantidade de máquinas ligadas em série. Dessa forma, um fluxo unidirecional contínuo e ininterrupto de lotes de produtos passa através da série de máquinas. Os problemas de flowshop encontram aplicações na indústria automotiva, química, eletrônica, alimentícia, farmacêutica, têxtil, e em qualquer linha de produção onde uma rota definida por estágios em série para toda produção é a mesma.

O problema investigado nesta dissertação é baseado no trabalho de Mohammadi et al. (2010a) e consiste na determinação de uma programação de produção em um ambiente de produção do tipo flowshop, com uma demanda dinâmica determinística em um horizonte de planejamento finito. São consideradas restrições de capacidade de produção, estoques de produtos intermediários e acabados, tempos de preparação de máquinas dependentes da sequência de produtos, preparações de máquinas mantidas entre períodos ( setup carryover). Dessa forma, decisões de dimensionamento de lotes e sequenciamento dos produtos têm de ser tomadas buscando programações de produção que minimizem o custo total de produção definido pelos custos de processamento de cada produto em cada estágio, o custo de preparação de máquinas e os custos relativos a estoque de produtos acabados e inacabados.

O problema proposto foi abordado de forma heurística, por meio de uma metaheurística denominada Times Assíncronos (A-Teams). A arquitetura do método Times Assíncronos faz com que agentes heurísticos atuem de forma concomitante em memórias de soluções, unindo-se assincronamente por meio de diferentes estratégias, visando encontrar soluções factíveis para então melhorá-las. O método A-Team foi proposto por de Souza e Talukdar (1993). A técnica é bem utilizada na solução de problemas combinatórios. A qualidade da heurística foi avaliada comparando os resultados obtidos com os resultados de métodos da literatura.

Em resumo, as contribuições desta dissertação são: 1) a análise de formulações matemáticas para o problema em questão, 2) a discussão acerca de limitantes inferiores para o problema, 3) o desenvolvimento de diversas heurísticas para o problema tratado neste trabalho e 4) a comparação dos procedimentos desenvolvidos com procedimentos da literatura.

O restante da dissertação é organizada da seguinte forma: no Capítulo 2 é apresentado uma revisão sobre o problema integrado de programação da produção sob o contexto de um ambiente de produção flowshop. No Capítulo 3, o problema abordado neste trabalho é definido e discutido. No Capítulo 4 os limitantes inferiores e as heurísticas da literatura são apresentados. No Capítulo 5, a abordagem metaheurística Times Assíncronos é apresen- 
tada. No Capítulo 6, os experimentos computacionais e seus resultados são apresentados. Por fim, o Capítulo 7 apresenta as conclusões do trabalho seguidas de direções futuras de pesquisa. Ao final, os Apêndices A e B discutem técnicas gráficas de apresentação de resultados e os Apêndices C e D apresentam todos os resultados computacionais. 


\section{Revisão da Literatura}

O planejamento de produção é o planejamento da aquisição de recursos e matériaprima, assim como o planejamento das atividades produtivas, necessárias para transformar matéria-prima em produtos finais atendendo a demanda de consumidores da maneira mais eficiente e econômica. Em ambiente industriais, os problemas abordados necessitam de decisões de dimensionamento de lotes de produção de distintos produtos a serem fabricados ou processados (lot sizing), decisões acerca do tempo necessário para a produção de cada um desses lotes (scheduling) e decisões de sequenciamento dos lotes de produção (sequencing). O objetivo é geralmente atender a demanda com um custo mínimo. Esses problemas são tipicamente de curto a médio prazo, ou problemas de planejamento operacional a tático (POCHET; WOLSEY, 2006).

O problema de dimensionamento de lotes $(P D L)$ é um problema clássico de planejamento de produção, e consiste em determinar o tamanho de lotes a serem produzidos, assim como a data dessa produção. Essa produção está inserida num horizonte de planejamento (planning horizon) e existe uma demanda de produtos a ser atendida. O objetivo é geralmente de cunho econômico, simulando custos de produção. Estes custos são tradicionalmente divididos em custos de estoque (inventory holding costs) e custos de produção (production costs), divididos em custos de processamento de tarefas (processing costs) e custos de preparação de máquinas (setup costs). Na literatura muitos trabalhos abordam o tema, dentre eles, Bodt et al. (1984), Bahl et al. (1987), Kuik et al. (1994), Drexl e Kimms (1997), Karimi et al. (2003), Brahimi et al. (2006), Quadt e Kuhn (2007), Jans e Degraeve $(2007,2008)$ são boas referências para estudo do problema, seu histórico, métodos de solução, distintos modelos e suas extensões.

Historicamente, o trabalho de Harris (1913) foi um dos precursores dessa área, com o conceito de Economic Order Quantity. De acordo com (BAHL et al., 1987), o estudo apresentado por Harris (1913) é a primeira formulação quantitativa para dimensionamento de lotes. As condições desse problema são bastante simples, pois são considerados uma taxa de demanda constante e parâmetros de custos de produção estáticos num horizonte 
de planejamento infinito. Para problemas de dimensionamento de lotes cuja demanda é dinâmica, um importante trabalho é relacionado a Wagner e Whitin (1958), que abordam o problema de dimensionamento de lotes de um único item e custos de produção dinâmicos. Os autores deste trabalho determinam por meio de programação dinâmica uma solução ótima para o problema. Porém, ambos trabalhos não consideram uma importante característica do sistema produtivo, as restrições de capacidade. O trabalho de Manne (1958) é um dos precursores do PDL que consideram restrições de capacidade e possui o objetivo de minimizar o uso de horas extras (overtime). A adição da restrição de capacidade torna o problema NP-Difícil de acordo com o trabalho de Bitran e Yanasse (1982).

Um aspecto fundamental do problema de dimensionamento de lotes reside na produção de distintos produtos num mesmo ambiente de produção. Consequentemente, as máquinas designadas a efetuar determinada tarefa devem estar preparadas para isso. A preparação das máquinas (setup) pode acarretar em custos (setup costs) e consumo da capacidade, geralmente tempo (setup times). As preparações de máquinas ainda podem ser dependentes ou independentes da sequência dependendo dos produtos e do ambiente de produção. Nos últimos anos, foi adicionado aos modelos matemáticos a possibilidade de preservar a preparação de máquina entre períodos, assim, uma máquina pode iniciar a produção em um período sem necessidade de preparação. Este procedimento é conhecido na literatura como preservação de preparação (setup carryover) e aproxima os modelos à realidade de linhas de produção ininterruptas ou mesmo aquelas que as máquinas estão prontas assim que iniciadas. Trabalhos que consideram essa característica são: Gopalakrishnan et al. (1995), Sox e Gao (1999), Gopalakrishnan et al. (2000, 2001), Porkka et al. (2003), Suerie e Stadler (2003), Karimi et al. (2006), Briskorn (2006) e Sung e Maravelias (2008).

Com o tempo, os modelos matemáticos de dimensionamento de lotes vão incorporando mais e mais aspectos de programação de produção. Esses modelos essencialmente determinam o início e fim das tarefas (programação - scheduling), a ordem na qual são processados (sequenciamento - sequencing) e a designação das tarefas às máquinas (carregamento - loading) (JANS; DEGRAEVE, 2008).

Problemas de sequenciamento são muito comuns. Eles existem sempre quando há uma decisão acerca da ordem que um determinado número de tarefas possam ser realizadas (CONWAY et al., 1967). No contexto desta dissertação, o problema de sequenciamento consiste em determinar a ordem das operações a serem efetuadas em uma máquina. Na 
literatura, alguns autores consideram a diferença entre scheduling e sequencing tão sutil que são considerados sinônimos, porém essa consideração não é compartilhada neste documento. O problema de sequenciamento é bem discutido em Conway et al. (1967), Ashour (1972), Baker (1974), Graham et al. (1979), Lawler et al. (1993) e Pinedo (2008).

O problema de programação da produção (production scheduling ou simplesmente scheduling) é um processo de tomada de decisões muito utilizado por indústrias de bens e serviços. Este problema trata da alocação de recursos a tarefas dentro de períodos de tempo e sua finalidade é otimizar um ou mais objetivos (PINEDO, 2008). Devido a sua aplicação direta na indústria, a teoria de scheduling é muito estudada na literatura, podendo ser vista em Conway et al. (1967), Tanaev et al. (1994), Pinedo (2008) e Baker e Trietsch (2009).

O problema de programação da produção é dependente do ambiente de produção (shop), já que os tempos de cada unidade de um determinado lote dependem da sua rota através dos estágios de produção, da disponibilidade desses estágios e dos tempos de outras unidades do mesmo lote ou mesmo de outros produtos. Existem vários tipos de linhas de produção definidos de acordo com a configuração das máquinas e a rota dos produtos nas máquinas. Em Pinedo (2008) os ambientes de produção são classificados como única máquina, máquinas paralelas, flowshop, jobshop e openshop e suas respectivas variações híbridas flowshop híbrido (flowshop flexível) e jobshop híbrido (jobshop flexível). A linha de produção correspondente a uma máquina implica que todas as tarefas devem ser alocadas nesta única máquina. Para ambientes de produção compostos de máquinas paralelas, estas podem ser de três tipos, idênticas, uniformes ou não-relacionadas. O ambiente de produção flowshop ou simplesmente flowshop é caracterizado por lotes de produtos sendo produzidos por uma quantidade de máquinas ligadas em série. Dessa forma, um fluxo unidirecional contínuo e ininterrupto de lotes de produtos passa através da série de máquinas. No ambiente jobshop cada produto possui sua própria rota de produção predeterminada, sendo possível até mesmo ser processado por um mesmo estágio de produção mais de uma vez (recirculation). No ambiente openshop não há restrições às rotas das tarefas nas máquinas. Os ambientes híbridos são aqueles que cada estágio de produção pode ser formado por máquinas em paralelo. No contexto desta dissertação, o ambiente de produção estudado é flowshop.

O primeiro problema de flowshop scheduling ( FSP), resolvido polinomialmente por Johnson (1954), tratava de um problema de programação da produção com duas máquinas e com o objetivo de minimizar o instante de término da produção (makespan). Para um 
número de máquinas maior que dois, o problema é NP-Difícil, de acordo com Garey et al. (1976). Cada estágio da linha produtiva pode possuir uma sequência diferente de produção, caracterizando o problema de flowshop não-permutacional. Porém é uma prática industrial e da literatura manter a mesma sequência para todos os estágios de produção, definido como flowshop permutacional. Esta prática é justificada pela restrição no número de programações possíveis, já que se considerada uma linha de produção nãopermutacional de $M$ máquinas e $N$ produtos distintos existem $(N !)^{M}$ programações para o caso não-permutacional e apenas $N$ ! programações para o caso permutacional.

Em Potts et al. (1991) os autores provam que a diferença entre programações permutacionais e não permutacionais pode variar por um fator maior que $\frac{1}{2} \sqrt{M}$, sendo $M$ o número de máquinas, para o problema de flowshop com o objetivo de minimizar o instante de término da produção makespan, uma medida amplamente utilizada na área, pois ela sugere uma utilização eficiente de todas as máquinas (PINEDO, 2008).

Algumas características do problema de flowshop scheduling foram amplamente estudadas. Allahverdi et al. (1999), Cheng et al. (2000) revisaram o problema de flowshop munido de preparações de máquinas. Para o problema de flowshop scheduling, Gupta e Stafford Jr. (2006) fazem uma revisão histórica em comemoração aos 50 anos desde o artigo de Johnson (1954), agrupando os artigos cronologicamente, descrevendo as características das publicações científicas de cada uma delas, apresentando os estágios da pesquisa atual e futuras áreas de pesquisa. De acordo com este trabalho, a partir da década de 90, problemas de programação de produção envolvendo decisões integradas de dimensionamento e sequenciamento de lotes se tornaram mais frequentes. Até então a literatura de scheduling geralmente assumia que as decisões de dimensionamento de lotes já estavam decididas, enquanto os problemas de dimensionamento de lotes desconsideravam ou aproximavam os detalhes de sequenciamento.

Focando no problema integrado de programação da produção com dimensionamento e sequenciamento de lotes, e mais ainda, visando problemas do ambiente de produção flowshop, alguns trabalhos serão discutidos adiante. O problema integrado de programação de produção é abordado por Potts e Wassenhove (1992) para todo ambiente de produção. Os autores definem as decisões de batching como o agrupamento de produtos similares e de lot sizing como a divisão de um lote em sublotes, também conhecido na literatura como lot splitting. Essa quebra do lote em sublotes para sistemas de produção com múltiplos estágios faz com que partes do mesmo lote de produtos possam ser processados ao mesmo tempo em estágios diferentes, o que caracteriza o lot streaming. 
Drexl e Kimms (1997) apresentam formulações de programação inteira mista focando o problema de dimensionamento de lotes. A partir do modelo clássico de dimensionamento de lotes com restrições de capacidade, são discutidas abordagens que subdividem os períodos em microperíodos (small bucket problems), abordagens de uso do tempo contínuo, com tempos de entrega para os itens e modelos que envolvem relações multiestágio, não focando em nenhum ambiente de produção.

Zhu e Wilhelm (2006) apresentam uma revisão de problemas que combinam lot sizing e scheduling com preparações de máquinas dependentes da sequência. Os problemas são categorizados por ambiente de produção e por abordagem de solução. Neste trabalho de Zhu e Wilhelm (2006), as referências do problema integrado de lot sizing e scheduling para problemas com múltiplos estágios são escassas, e há apenas três referências envolvendo máquinas paralelas, três para flowshop e nenhuma referência de jobshop com decisões integradas de dimensionamento e sequenciamento de lotes. As três referências de flowshop são Sikora et al. (1996), Sikora (1996) e Lee et al. (1997) e serão discutidas adiante.

Sikora et al. (1996) consideram um problema de dimensionamento de lotes em um ambiente flowshop com estoques intermediários, preparações de máquinas dependentes da sequência e restrições de capacidade, em uma fábrica de placas de circuito impresso. Os autores foram pioneiros em tratar este tipo de ambiente de produção com uma abordagem integrada dos problemas de dimensionamento e sequenciamento de lotes. A proposta desenvolvida foi uma heurística denominada Integrated Approach resultado da fusão adaptada de duas heurísticas, a de Silver e Meal (1973) que trata do dimensionamento de lotes e a de Palmer (1965) para o sequenciamento dos lotes. O objetivo foi minimizar o makespan dos períodos e os custos de estoque. Para o mesmo problema, Sikora (1996) propõe um algoritmo genético, obtendo melhores resultados em menor tempo, por meio de uma aproximação no recálculo do makespan, além do uso de seleção por torneio, agentes de mutação e cruzamento específicos para o dimensionamento e sequenciamento de lotes. Sikora (1996) compara os seus resultados com a heurística Integrated Approach e mostra que os resultados do algoritmo genético foram melhores. Em Lee et al. (1997) os autores desenvolveram uma metaheurística híbrida para o problema de Sikora (1996), baseada em algortimos genéticos e simulated annealing. Os autores também consideram a divisão de lotes (lot splitting), assim no mesmo período são permitidos o processamento de vários lotes diferentes do mesmo produto. Assim como Lee et al. (1997), Ponnambalam e Reddy (2003) desenvolveram uma heurística híbrida, composta de algoritmo genético e simulated annealing para o problema de Sikora (1996). O algoritmo genético trata do dimensionamento de lotes, enquanto o simulated annealing faz buscas locais do sequenciamento. 
Os agentes de mutação e de cruzamento do algoritmo genético são os mesmos de Sikora (1996) e não tratam soluções com lot splitting. Belo Filho et al. (2009) apresentam uma abordagem metaheurística utilizando a técnica Times Assíncronos para o mesmo problema de Sikora (1996). Os autores comparam os resultados com as técnicas de Sikora (1996) e Ponnambalam e Reddy (2003), obtendo bons resultados para o problema. Vale ressaltar que nenhum destes trabalhos apresentou um modelo matemático para o problema.

Modelos matemáticos para problemas de flowshop com decisões integradas de dimensionamento e sequenciamento de lotes são escassos, devido à complexidade do problema. Smith-Daniels e Ritzman (1988) apresentam modelos de programação inteira mista para o problema de flowshop envolvendo um horizonte de planejamento finito, permitindo famílias de produtos, dimensionamento de lotes, estoques de itens intermediários, setup dependente da sequência e setup carryover. Devido a enorme quantidade de variáveis no modelo matemático, este é útil apenas para resolver instâncias pequenas. Dauzère-Péres e Lasserre (1994) desenvolvem um modelo de dimensionamento e de sequenciamento de lotes integrado para a linha de produção jobshop. O problema de flowshop é um caso específico de jobshop, em que todos os itens possuem a mesma sequência de operações para ser finalizado. Não são considerados preparação de máquinas e estoques intermediários.

Recentemente, o trabalho de Mohammadi et al. (2008) apresentou o modelo matemático de programação inteira mista (Mixed Integer Program - MIP) utilizado nesta dissertação, combinando decisões de dimensionamento de lotes e de programação de produção para flowshop considerando preparações de máquinas dependentes da sequência, preservação de preparação e programações de produção não-permutacionais. Os autores desenvolveram dois limitantes inferiores, que, para pequenas instâncias, foram comparados com a solução ótima do MIP. Quatro heurísticas foram apresentadas, duas delas são heurísticas baseadas na estratégia de horizonte rolante, enquanto as outras são heurísticas do tipo relax-and-fix. As técnicas utilizadas nestas heurísticas são detalhadas ao longo desta dissertação. Algumas dessas heurísticas desconsideram soluções cuja programação de produção é não-permutacional.

O trabalho de Mohammadi et al. (2010a) é uma extensão do artigo de Mohammadi et al. (2008), onde os autores abordam o mesmo modelo, os mesmos limitantes inferiores e desenvolvem algumas heurísticas relax-and-fix e baseadas na estratégia de horizonte rolante. Todas as heurísticas são baseadas no problema inteiro-misto. Da mesma maneira que o artigo anterior, há heurísticas que consideram programações de produção não-permutacionais e aquelas onde apenas soluções permutacionais são consideradas. A 
consideração da permutabilidade torna as heurísticas capazes de resolver um número de instâncias maior.

O trabalho de Mohammadi et al. (2010b) considera o mesmo MIP de Mohammadi et al. (2008), porém duas novas heurísticas são apresentadas. Ambas utilizam a estratégia de horizonte rolante, consideram apenas soluções permutacionais e determinam que a produção de todas as máquinas é a mesma para qualquer período. Assim, o número de variáveis e de restrições é diminuído, o que torna as heurísticas mais eficientes. As duas heurísticas são construtivas e se baseam no procedimento heurístico utilizado por Nawaz et al. (1983), uma heurística bem conhecida da literatura de flowshop, mas que não considera o dimensionamento de lotes. 


\section{Definição e Modelagem do Problema}

O problema considerado nesta dissertação está situado num ambiente de produção do tipo flowshop, onde máquinas dispostas em série transformam matérias-primas em diversos produtos acabados. Decisões de sequenciamento e dimensionamento de lotes devem ser tomadas com o objetivo de uma produção eficiente e econômica. Desta forma, deve-se determinar a quantidade de produção de um produto em cada máquina e em cada período, assim como a sequência de produtos a serem processados em cada máquina, obtendo a partir desses dados uma programação de produção. Esta programação da produção deve satisfazer algumas condições, apresentadas adiante. Assume-se que:

- Produtos (itens) diferentes são produzidos pela linha de produção;

- Os produtos são independentes uns dos outros;

- O horizonte de planejamento é finito e dividido em períodos;

- Os estágios de produção (máquinas) estão dispostos em série;

- Cada máquina pode processar apenas um lote de algum produto ao mesmo tempo;

- Cada máquina é única no sentido de que seu processo ocupa a mesma posição na sequência de processos de transformação da matéria-prima ao produto final;

- Todas as máquinas possuem restrições de capacidade;

- Tempos e custos de preparação de máquina dependentes da sequência ocorrem ao se fazer uma troca de produção nas máquinas (setup);

- O setup de uma máquina deve iniciar e terminar no mesmo período (não se considera setup crossover); 
- Os tempos e custos de preparação de máquina respeitam a desigualdade triangular, ou seja, o tempo (ou custo) de preparação de uma máquina de um produto para outro é sempre menor que utilizar setups de produtos intermediários;

- Não é necessária a preparação de máquina para tarefas de mesmo produto;

- As máquinas preservam sua preparação entre os períodos (setup carryover);

- No início do horizonte de planejamento as máquinas estão preparadas para um determinado produto;

- Para cada período é permitido $N$ (número de produtos) preparações de máquina, que significa que a sequência de produção de cada máquina possui tamanho $N$ (pode admitir lotes do mesmo produto no mesmo período);

- Há uma demanda externa apenas para produtos acabados (processados pelo último estágio produtivo);

- A demanda é sempre atendida (não há atrasos);

- Entre duas máquinas consecutivas há um estoque intermediário irrestrito onde produtos processados pela primeira máquina aguardam o início do processo na máquina seguinte;

- O tempo de transporte de produtos intermediários e outros processos entre as máquinas sucessivas é desprezível;

- Não há perda de produção, seja por perecibilidade ou por eventos não previstos;

- No início do planejamento, todos os recursos requeridos estão disponíveis, especialmente para a primeira máquina;

- Para as demais máquinas, o processamento de um lote só se inicia se todos os recursos necessários estão disponíveis para produção, ou seja, se todo lote já foi processado pela máquina anterior e está no estoque intermediário;

- Não há prioridade de produção nas máquinas entre produtos (preemption).

Para simular o tempo de espera (idleness) da máquina para produção, utiliza-se a abordagem de Fandel e Stammen-Hegene (2006), denominada produto fantasma (shadow product). Os tempos de processamento do produto fantasma são iguais aos tempos de processamento do produto original. Porém, a quantidade de produto fantasma existente 
é variável, dependendo exclusivamente do tempo de espera da máquina para produção de determinado lote. Não há demanda para produtos fantasmas e nenhum custo é associado a existência deles.

Para os modelos matemáticos apresentados nesta dissertação, os índices, parâmetros e variáveis de decisão utilizados foram:

$$
\begin{aligned}
\text { Índices } & \\
i, j, k & \text { Tipos de produtos } \\
n, n^{\prime}, n^{\prime \prime} & \text { Posição na sequência de produção } \\
m & \text { Máquinas } \\
t & \text { Períodos }
\end{aligned}
$$

Parâmetros

$T$ Horizonte de planejamento

$N \quad$ Número de produtos diferentes

M Número de Máquinas

bigM Um número grande

$C_{m t}$ Capacidade (tempo disponível) da máquina $m$ no período $t$

$d_{j t}$ Demanda externa do produto $j$ no período $t$

$h_{j m}$ Custo de estoque do produto $j$ na máquina $m$

$b_{j m}$ Tempo de produção do produto $j$ na máquina $m$

$p_{j m t}$ Custo de produção do produto $j$ na máquina $m$ no período $t$

$S_{i j m}$ Tempo de preparação de máquina do produto $i$ ao produto $j$ na máquina $m$

$W_{i j m}$ Custo de preparação de máquina do produto $i$ ao produto $j$ na máquina $m$

$j 0_{m} \quad$ Configuração inicial de preparação da máquina $m$

Variáveis

$I_{j m t}$ Estoque do produto $j$ após processo na máquina $m$ ao final do período $t$

$x_{j m t}^{n} \quad$ Quantidade do produto $j$ processada na máquina $m$ no período $t$, na $n$-ésima posição da sequência deste período

$q_{j m t}^{n} \quad$ Tempo de espera para a produção do lote $x_{j m t}^{n}$ por meio do uso de shadow product

$y_{i j m t}^{n} \begin{cases}1 & \text { Se o } n \text {-ésimo setup na máquina } m \text { do período } t \text { do produ- } \\ & \text { to } i \text { ao produto } j \text { é requerido } \\ 0 & \text { Caso contrário }\end{cases}$ 
O modelo matemático a seguir foi proposto por Mohammadi et al. (2010a), e será também denominado como $M M O$ (modelo matemático original).

$$
\operatorname{Min} \sum_{n=1}^{N} \sum_{j=1}^{N} \sum_{i=1}^{N} \sum_{m=1}^{M} \sum_{t=1}^{T} W_{i j m} \cdot y_{i j m t}^{n}+\sum_{n=1}^{N} \sum_{j=1}^{N} \sum_{m=1}^{M} \sum_{t=1}^{T} p_{j m t} \cdot x_{j m t}^{n}+\sum_{j=1}^{N} \sum_{m=1}^{M} \sum_{t=1}^{T} h_{j m} \cdot I_{j m t}
$$

Sujeito a:

$$
\begin{aligned}
& d_{j t}=I_{j M t-1}+\sum_{n=1}^{N} x_{j M t}^{n}-I_{j M t} \quad j=1, \ldots, N, t=1, \ldots, T \\
& I_{j m t-1}+\sum_{n=1}^{N} x_{j m t}^{n}=I_{j m t}+\sum_{n=1}^{N} x_{j m+1 t}^{n} \\
& j=1, \ldots, N, m=1, \ldots, M-1, t=1, \ldots, T \\
& \operatorname{big} M \cdot\left(\sum_{i=1, i \neq j\left(n^{\prime}>1\right)}^{N} y_{i j m t}^{n^{\prime}}-1\right)+\sum_{n=1}^{n^{\prime}} \sum_{i=1}^{N} \sum_{k=1}^{N} S_{i k m} \cdot y_{i k m t}^{n}+ \\
& \sum_{n=1}^{n^{\prime}} \sum_{k=1}^{N} b_{k m} \cdot q_{k m t}^{n}+\sum_{n=1}^{n^{\prime}} \sum_{k=1}^{N} b_{k m} \cdot x_{k m t}^{n} \leq \\
& \operatorname{big} M \cdot\left(1-\sum_{i=1, i \neq j\left(n^{\prime \prime}>1\right)}^{N} y_{i j m+1 t}^{n^{\prime \prime}}\right)+\sum_{n=1}^{n^{\prime \prime}} \sum_{i=1}^{N} \sum_{k=1}^{N} S_{i k m+1} \cdot y_{i k m+1 t}^{n}+ \\
& \sum_{n=1}^{n^{\prime \prime}} \sum_{k=1}^{N} b_{k m+1} \cdot q_{k m+1 t}^{n}+\sum_{n=1}^{n^{\prime \prime}-1} \sum_{k=1}^{N} b_{k m+1} \cdot x_{k m+1 t}^{n} \\
& j=1, \ldots, N, n^{\prime}=1, \ldots, N, n^{\prime \prime}=1, \ldots, N, m=1, \ldots, M-1, t=1, \ldots, T \\
& \sum_{n=1}^{N} \sum_{i=1}^{N} \sum_{j=1}^{N} S_{i j m} \cdot y_{i j m t}^{n}+\sum_{n=1}^{N} \sum_{j=1}^{N} b_{j m} \cdot q_{j m t}^{n}+\sum_{n=1}^{N} \sum_{j=1}^{N} b_{j m} \cdot x_{j m t}^{n} \leq C_{m t} \\
& m=1, \ldots, M, t=1, \ldots, T \\
& x_{j m t}^{n} \leq\left(\frac{C_{m t}}{b_{j m}}\right) \sum_{i=1, i \neq j(n>1)}^{N} y_{i j m t}^{n} \\
& n=1, \ldots, N, j=1, \ldots, N, m=1, \ldots, M, t=1, \ldots, T \\
& q_{j m t}^{n} \leq\left(\frac{C_{m t}}{b_{j m}}\right) \sum_{i=1}^{N} y_{i j m t}^{n} \\
& n=1, \ldots, N, j=1, \ldots, N, m=1, \ldots, M, t=1, \ldots, T \\
& y_{j i m 1}^{1}=0 \quad j \neq j 0_{m}, i=1, \ldots, N, m=1, \ldots, M
\end{aligned}
$$




$$
\begin{gathered}
\sum_{i=1}^{N} y_{j 0_{m} i m 1}^{1}=1 \quad m=1, \ldots, M \\
\sum_{j=1}^{N} y_{j i m t}^{n}=\sum_{k=1}^{N} y_{i k m t}^{n+1} \\
n=1, \ldots, N-1, i=1, \ldots, N, m=1, \ldots, M, t=1, \ldots, T \\
\sum_{j=1}^{N} y_{j i m t-1}^{N}=\sum_{k=1}^{N} y_{i k m t}^{1} \quad \begin{array}{c}
i=1, \ldots, N, m=1, \ldots, M, t=2, \ldots, T \\
y_{i j m t}^{n} \in\{0,1\} \\
I_{j m t}, x_{j m t}^{n}, q_{j m t}^{n} \geq 0 \\
j=1, \ldots, N, m=1, \ldots, M
\end{array}
\end{gathered}
$$

A função 3.1 introduz a função objetivo do problema, que representa os custos de produção dessa linha de produção. Os custos envolvidos são custos de preparações de máquinas, de produção e de estoque. As equações 3.2 e 3.3 representam o fluxo de produção entre as máquinas, culminando na satisfação da demanda (3.2), sem atrasos.

As restrições 3.4 garantem que um produto só pode ser processado em determinada máquina se já foi previamente processado na máquina anterior, ou seja, garantem a interação vertical entre as máquinas. O lado esquerdo da restrição 3.4 representa o instante de término do processamento do $n$-ésimo produto na máquina $m$, enquanto o lado direito indica que o instante de início do processamento do mesmo lote na máquina $m+1$ (que pode estar em uma posição da sequência de produção da máquina diferente). As parcelas da restrição relacionadas ao big $M$ são utilizadas para ativar ou não a restrição, e esta só é ativada quando $y_{i j m t}^{n^{\prime}}=1$ e $y_{i j m t}^{n^{\prime \prime}}=1$. Por exemplo, na Figura 1, um gráfico de Gantt ilustrando uma programação de produção é apresentado. Mais informações acerca desse tipo de representação estão no Apêndice A. Neste exemplo, três tarefas são processadas nas máquinas $M 1$ e $M 2$. No gráfico de Gantt, a parte mais escura representa os tempos de setup. As partes coloridas da barra são os tempos de processamento dos lotes. A restrição 3.4 garante que qualquer produto será processado na máquina $M 2$ após seu processo na máquina $M 1$, por mais que a sequência de produção das máquinas $M 1$ e $M 2$ sejam diferentes.

As restrições 3.5 limitam o conjunto de soluções impondo uma capacidade máxima para cada máquina em cada período. As restrições 3.6 limitam a existência de determinado lote à existência da preparação de máquina correspondente. Note que, exceto para a primeira posição, a existência do lote também necessita da ocorrência de um setup não- 


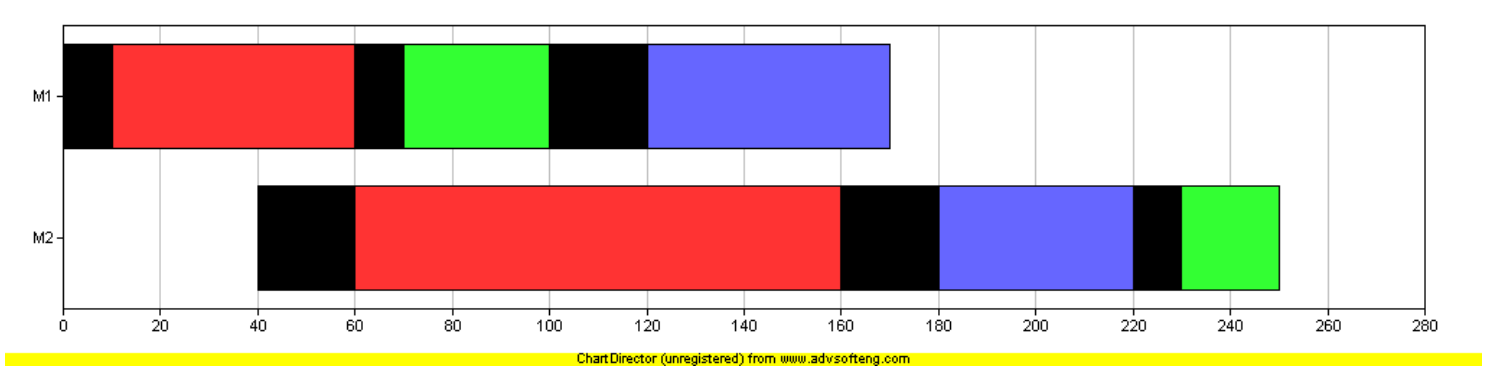

Figura 1: Diagrama de Gantt representando interação vertical.

nulo (que envolve a mudança da configuração da máquina). As restrições 3.7 são similar às restrições anteriores e também limitam o tempo de espera (quantidade de produto fantasma) à existência da preparação de máquina.

As restrições 3.8 e 3.9 impõem que a primeira preparação de máquina do primeiro período seja do produto no qual a máquina já está preparada para outro produto, e que qualquer outra configuração de setup é nula. As restrições 3.10 e 3.11 estão relacionadas ao sequenciamento dos produtos por meio da limitação entre setups sucessivos, inclusive no caso da preservação da preparação de máquina. As restrições 3.12 e 3.13 indicam o tipo das variáveis de decisão. Por último, não há estoque no início do horizonte de planejamento, como define a expressão 3.14.

\subsection{Modelo Matemático para o Caso Permutacional}

O problema anterior em um ambiente de produção flowshop permutacional assume que a sequência de produção em todas as máquinas é a mesma. Assim, restringe-se o número de soluções com relação ao problema em flowshop puro. Algumas alterações no modelo matemático de programação inteira mista anterior devem ser feitas para adaptação do mesmo. A primeira delas é que as variáveis binárias de setup, $y_{i j m t}^{n}$, podem ser reduzidas a $y_{i j t}^{n}$, pois a sequência é indiferente em todas as máquinas. Assim, o número de variáveis binárias é reduzido de $N^{3} M T$ para $N^{3} T$. O parâmetro $j 0_{m}$ também é reduzido a $j 0$, deixando todas as máquinas preparadas para o mesmo produto no início do horizonte de planejamento.

Uma adaptação nas restrições 3.4, responsável pela interação vertical, também é efetuada resultando na restrição 3.18. A restrição 3.4 é simplificada pois é desnecessária sua ativação devido a possíveis trocas de sequência de produção entre as máquinas (conforme restrição 3.4). O número de restrições de interação vertical é reduzido da ordem de $O\left(N^{3} M T\right)$ para $O(N M T)$, o que deixa o modelo matemático menor. As restrições 
restantes são análogas e a função objetivo é mantida, o que torna as soluções dos modelos matemáticos comparáveis.

O modelo matemático é original do trabalho de Mohammadi et al. (2010a) e aqui é denominado como MMP (modelo matemático permutacional). O MIP resultante é descrito adiante:

$$
\operatorname{Min} \sum_{n=1}^{N} \sum_{j=1}^{N} \sum_{i=1}^{N} \sum_{m=1}^{M} \sum_{t=1}^{T} W_{i j m} \cdot y_{i j t}^{n}+\sum_{n=1}^{N} \sum_{j=1}^{N} \sum_{m=1}^{M} \sum_{t=1}^{T} p_{j m t} \cdot x_{j m t}^{n}+\sum_{j=1}^{N} \sum_{m=1}^{M} \sum_{t=1}^{T} h_{j m} \cdot I_{j m t}
$$

Sujeito a:

$$
\begin{aligned}
& d_{j t}=I_{j M t-1}+\sum_{n=1}^{N} x_{j M t}^{n}-I_{j M t} \quad j=1, \ldots, N, t=1, \ldots, T \\
& I_{j m t-1}+\sum_{n=1}^{N} x_{j m t}^{n}=I_{j m t}+\sum_{n=1}^{N} x_{j m+1 t}^{n} \\
& j=1, \ldots, N, m=1, \ldots, M-1, t=1, \ldots, T \\
& \sum_{n=1}^{n^{\prime}} \sum_{i=1}^{N} \sum_{j=1}^{N} S_{i j m} \cdot y_{i j t}^{n}+\sum_{n=1}^{n^{\prime}} \sum_{j=1}^{N} b_{j m} \cdot q_{j m t}^{n}+\sum_{n=1}^{n^{\prime}} \sum_{j=1}^{N} b_{j m} \cdot x_{j m t}^{n} \leq \\
& \sum_{n=1}^{n^{\prime}} \sum_{i=1}^{N} \sum_{j=1}^{N} S_{i j m+1} \cdot y_{i j t}^{n}+\sum_{n=1}^{n^{\prime}} \sum_{j=1}^{N} b_{j m+1} \cdot q_{j m+1 t}^{n}+\sum_{n=1}^{n^{\prime}-1} \sum_{j=1}^{N} b_{j m+1} \cdot x_{j m+1 t}^{n} \\
& n^{\prime}=1, \ldots, N, m=1, \ldots, M-1, t=1, \ldots, T \\
& \sum_{n=1}^{N} \sum_{i=1}^{N} \sum_{j=1}^{N} S_{i j m} \cdot y_{i j t}^{n}+\sum_{n=1}^{N} \sum_{j=1}^{N} b_{j m} \cdot q_{j m t}^{n}+\sum_{n=1}^{N} \sum_{j=1}^{N} b_{j m} \cdot x_{j m t}^{n} \leq C_{m t} \\
& m=1, \ldots, M, t=1, \ldots, T \\
& x_{j m t}^{n} \leq\left(\frac{C_{m t}}{b_{j m}}\right) \sum_{i=1, i \neq j(n>1)}^{N} y_{i j t}^{n} \\
& n=1, \ldots, N, j=1, \ldots, N, m=1, \ldots, M, t=1, \ldots, T \\
& q_{j m t}^{n} \leq\left(\frac{C_{m t}}{b_{j m}}\right) \sum_{i=1}^{N} y_{i j t}^{n} \\
& n=1, \ldots, N, j=1, \ldots, N, m=1, \ldots, M, t=1, \ldots, T \\
& y_{j i 1}^{1}=0 \quad j \neq j 0, i=1, \ldots, N
\end{aligned}
$$




$$
\begin{gathered}
\sum_{i=1}^{N} y_{j 0 i 1}^{1}=1 \\
\sum_{j=1}^{N} y_{j i t}^{n}=\sum_{k=1}^{N} y_{i k t}^{n+1} n=1, \ldots, N-1, i=1, \ldots, N, t=1, \ldots, T \\
\sum_{j=1}^{N} y_{j i t-1}^{N}=\sum_{k=1}^{N} y_{i k t}^{1} \quad i=1, \ldots, N, t=2, \ldots, T \\
y_{i j t}^{n} \in\{0,1\} \\
I_{j m t}, x_{j m t}^{n}, q_{j m t}^{n} \geq 0 \\
j=1, \ldots, N, m=1, \ldots, M
\end{gathered}
$$

\subsection{Modelo Matemático Reduzido}

Para obter um modelo matemático mais reduzido, pode-se considerar que ao final de cada período apenas estoques de produtos acabados são permitidos. Isso implica que todas variáveis de estoque $I_{j m t}$ são reduzidas a $I_{j t}$, já que $I_{j m t}=0$, para todo $m<M$. Consequentemente, a dimensão dos lotes não varia de uma máquina para outra, para a mesma posição $n$, produto $j$ e período $t$. Dessa forma, as variáveis de decisão $x_{j m t}^{n}$ são simplificadas para $x_{j t}^{n}$. No entanto, as variáveis de decisão $q_{j m t}^{n}$, que representam o tempo de espera das máquinas, não são alteradas, pois cada máquina tem seu próprio tempo de espera para o processamento de cada produto.

Comparando com o modelo matemático imediatamente anterior, nota-se que as restrições 3.17, que garantem o fluxo de produção entre as máquinas é desnecessária, a partir da hipótese assumida de que não há estoques intermediários ao final do período. Alterações são necessárias também nas restrições 3.1, que antes limitavam o tamanho dos lotes de acordo com a máquina, agora limitam o tamanho do lote pela menor razão $\frac{C_{m t}}{b_{j m}}$, considerando todas as máquinas $(m=1, \ldots, M)$, dependendo do período e do produto analisado. As outras restrições são análogas e a função objetivo é mantida, assim os resultados podem ser comparados. O modelo matemático resultante é apresentado a seguir e será denominado como modelo matemático reduzido $(M M R)$, e foi apresentado por Mohammadi et al. (2010b).

$$
\operatorname{Min} \sum_{n=1}^{N} \sum_{j=1}^{N} \sum_{i=1}^{N} \sum_{m=1}^{M} \sum_{t=1}^{T} W_{i j m} \cdot y_{i j t}^{n}+\sum_{n=1}^{N} \sum_{j=1}^{N} \sum_{m=1}^{M} \sum_{t=1}^{T} p_{j m t} \cdot x_{j t}^{n}+\sum_{j=1}^{N} \sum_{t=1}^{T} h_{j M} \cdot I_{j t}
$$


Sujeito a:

$$
\begin{aligned}
& d_{j t}=I_{j t-1}+\sum_{n=1}^{N} x_{j t}^{n}-I_{j t} \quad j=1, \ldots, N, t=1, \ldots, T \\
& \sum_{n=1}^{n^{\prime}} \sum_{i=1}^{N} \sum_{j=1}^{N} S_{i j m} \cdot y_{i j t}^{n}+\sum_{n=1}^{n^{\prime}} \sum_{j=1}^{N} b_{j m} \cdot q_{j m t}^{n}+\sum_{n=1}^{n^{\prime}} \sum_{j=1}^{N} b_{j m} \cdot x_{j t}^{n} \leq \\
& \sum_{n=1}^{n^{\prime}} \sum_{i=1}^{N} \sum_{j=1}^{N} S_{i j m+1} \cdot y_{i j t}^{n}+\sum_{n=1}^{n^{\prime}} \sum_{j=1}^{N} b_{j m+1} \cdot q_{j m+1 t}^{n}+\sum_{n=1}^{n^{\prime}-1} \sum_{j=1}^{N} b_{j m+1} \cdot x_{j t}^{n} \\
& n^{\prime}=1, \ldots, N, m=1, \ldots, M-1, t=1, \ldots, T \\
& \sum_{n=1}^{N} \sum_{i=1}^{N} \sum_{j=1}^{N} S_{i j m} \cdot y_{i j t}^{n}+\sum_{n=1}^{N} \sum_{j=1}^{N} b_{j m} \cdot q_{j m t}^{n}+\sum_{n=1}^{N} \sum_{j=1}^{N} b_{j m} \cdot x_{j t}^{n} \leq C_{m t} \\
& m=1, \ldots, M, t=1, \ldots, T \\
& x_{j t}^{n} \leq\left(\min _{m=1, \ldots, M} \frac{C_{m t}}{b_{j m}}\right) \sum_{i=1, i \neq j(n>1)}^{N} y_{i j t}^{n} \\
& n=1, \ldots, N, j=1, \ldots, N, t=1, \ldots, T \\
& q_{j m t}^{n} \leq\left(\frac{C_{m t}}{b_{j m}}\right) \sum_{i=1}^{N} y_{i j t}^{n} \\
& n=1, \ldots, N, j=1, \ldots, N, m=1, \ldots, M, t=1, \ldots, T \\
& y_{j i 1}^{1}=0 \quad j \neq j 0, i=1, \ldots, N \\
& \sum_{i=1}^{N} y_{j 0 i 1}^{1}=1 \\
& \sum_{j=1}^{N} y_{j i t}^{n}=\sum_{k=1}^{N} y_{i k t}^{n+1} \quad n=1, \ldots, N-1, i=1, \ldots, N, t=1, \ldots, T \\
& \sum_{j=1}^{N} y_{j i t-1}^{N}=\sum_{k=1}^{N} y_{i k t}^{1} \quad i=1, \ldots, N, t=2, \ldots, T \\
& y_{i j t}^{n} \in\{0,1\} \\
& I_{j t}, x_{j t}^{n}, q_{j m t}^{n} \geq 0 \\
& I_{j 0}=0 \quad j=1, \ldots, N
\end{aligned}
$$

Os três modelos matemáticos de programação inteira mista ( $M M O, M M P$ e $M M R)$ podem ser utilizados para obter soluções do problema apresentado. Com relação ao espaço de soluções, todas as soluções do espaço de soluções de $M M R$ estão contidas no espaço 
de soluções de $M M P$, que por sua vez, estão contidas no espaço de soluções de $M M O$. Por $M M O$ ser um modelo mais abrangente, a sua resolução por meio de métodos exatos pode ser mais onerosa computacionalmente, assim, utilizando modelos matemáticos reduzidos, é possível obter boas soluções em tempo computacional aceitável, ou mesmo utilizar estas soluções como soluções iniciais para metaheurísticas. A seguir, um exemplo númerico é apresentado visando a melhor compreensão do problema e das diferenças entre as formulações matemáticas.

\subsection{Exemplo Numérico}

Um pequeno exemplo será apresentado nesta seção, com três produtos $(P 1, P 2, P 3)$, duas máquinas $(M 1, M 2)$ e dois períodos $(T 1, T 2)$. Os parâmetros são dados pelas Tabelas 1 a 7

Tabela 1: Demandas de Produtos.

\begin{tabular}{|c|ccc|}
\hline & $P 1$ & $P 2$ & $P 3$ \\
\hline$T 1$ & 150 & 40 & 120 \\
$T 2$ & 50 & 160 & 80 \\
\hline
\end{tabular}

Tabela 2: Tempos de processamento de produtos.

\begin{tabular}{|l|lll|}
\hline & $P 1$ & $P 2$ & $P 3$ \\
\hline$M 1$ & 1.8 & 1.5 & 2.0 \\
$M 2$ & 1.8 & 1.5 & 2.0 \\
\hline
\end{tabular}

Tabela 3: Custos de processamento de produtos.

\begin{tabular}{|l|ccc|ccc|}
\hline & \multicolumn{3}{|c|}{$M 1$} & \multicolumn{3}{c|}{$M 2$} \\
& $P 1$ & $P 2$ & $P 3$ & $P 1$ & $P 2$ & $P 3$ \\
\hline$T 1$ & 1.6 & 1.5 & 2.0 & 1.6 & 1.5 & 2.0 \\
$T 2$ & 2.0 & 1.5 & 2.0 & 2.0 & 1.5 & 2.0 \\
\hline
\end{tabular}

Tabela 4: Custos de estoque de produtos.

\begin{tabular}{|l|lll|}
\hline & $P 1$ & $P 2$ & $P 3$ \\
\hline$M 1$ & 0.2 & 0.4 & 0.2 \\
$M 2$ & 0.4 & 0.4 & 0.2 \\
\hline
\end{tabular}

A Tabela 1 apresenta a demanda de cada produto em cada período. As Tabelas 2 e 3 indicam os tempos e custos de processamento dos produtos na linha de produção, respectivamente. Os custos de estoque dos produtos são dados pela Tabela 4. As preparações de máquinas possuem tempos e custos quantificados, respectivamente, pelas Tabelas 5 e 
Tabela 5: Tempos de preparação dependentes da sequência.

\begin{tabular}{|c|ccc|ccc|}
\hline & \multicolumn{3}{|c|}{$M 1$} & \multicolumn{3}{c|}{$M 2$} \\
& $P 1$ & $P 2$ & $P 3$ & $P 1$ & $P 2$ & $P 3$ \\
\hline$P 1$ & 0 & 50 & 35 & 0 & 50 & 35 \\
$P 2$ & 40 & 0 & 50 & 40 & 0 & 50 \\
$P 3$ & 70 & 35 & 0 & 70 & 35 & 0 \\
\hline
\end{tabular}

Tabela 6: Custos de preparação dependentes da sequência.

\begin{tabular}{|c|ccc|ccc|}
\hline & \multicolumn{3}{|c|}{$M 1$} & \multicolumn{3}{c|}{$M 2$} \\
& $P 1$ & $P 2$ & $P 3$ & $P 1$ & $P 2$ & $P 3$ \\
\hline$P 1$ & 0 & 50 & 35 & 0 & 50 & 35 \\
$P 2$ & 40 & 0 & 50 & 40 & 0 & 50 \\
$P 3$ & 70 & 35 & 0 & 70 & 35 & 0 \\
\hline
\end{tabular}

Tabela 7: Capacidades das máquinas.

\begin{tabular}{|c|cc|}
\hline & $M 1$ & $M 2$ \\
\hline$T 1$ & 700 & 1000 \\
$T 2$ & 700 & 1000 \\
\hline
\end{tabular}

6. Por último, as capacidades das máquinas são dadas pela Tabela 7. Todas as máquinas estão previamente preparadas para o produto 1.

O exemplo numérico foi resolvido pelo software de otimização IBM ILOG CPLEX, versão 12.1, sob a formulação matemática $M M P$. Para este exemplo, duas soluções ótimas foram encontradas e estão ilustradas nas Figuras 2 e 3. Os intervalos na cor preta representam as preparações de máquinas realizadas, enquanto os intervalos coloridos representam o processamento de alguma tarefa. Os produtos 1,2,3 são representados pelas cores vermelha, verde e azul, respectivamente. Ambas soluções possuem as mesmas sequências de produção para todos os períodos, mas outras variáveis de decisão são diferentes. A sequência de produção do primeiro período de ambas soluções é iniciada pelo processamento do Produto 1, seguida dos Produtos 3 e 2 (azul e verde), enquanto que no segundo período sua sequência de produção inicia-se pelo Produto 2 (dado a preservação de preparação de máquina), continua com o Produto 1 (vermelho) e finaliza o planejamento com a produção do Produto 3, ou seja, as sequências de produção são $(1,3,2)$ e $(2,1,3)$, respectivamente, em todas as máquinas. As dimensões dos lotes, os estoques necessários e a presença de produtos fantasmas são apresentados a seguir, nas Tabelas 8 e 9 . As variáveis cujo valor é nulo foram ignoradas. Note que na primeira solução um estoque de produtos acabados $\left(I_{121}=25,00\right)$ é gerado enquanto que na segunda solução, parte do estoque gerado é acabado $\left(I_{121}=16,67\right)$ e parte do estoque é inacabado $\left(I_{111}=16,67\right)$, tendo de ser pro- 
cessado pela segunda máquina para assim atender a demanda. O valor da função objetivo das soluções ótimas resultam em um custo mínimo de 2360 unidades monetárias.
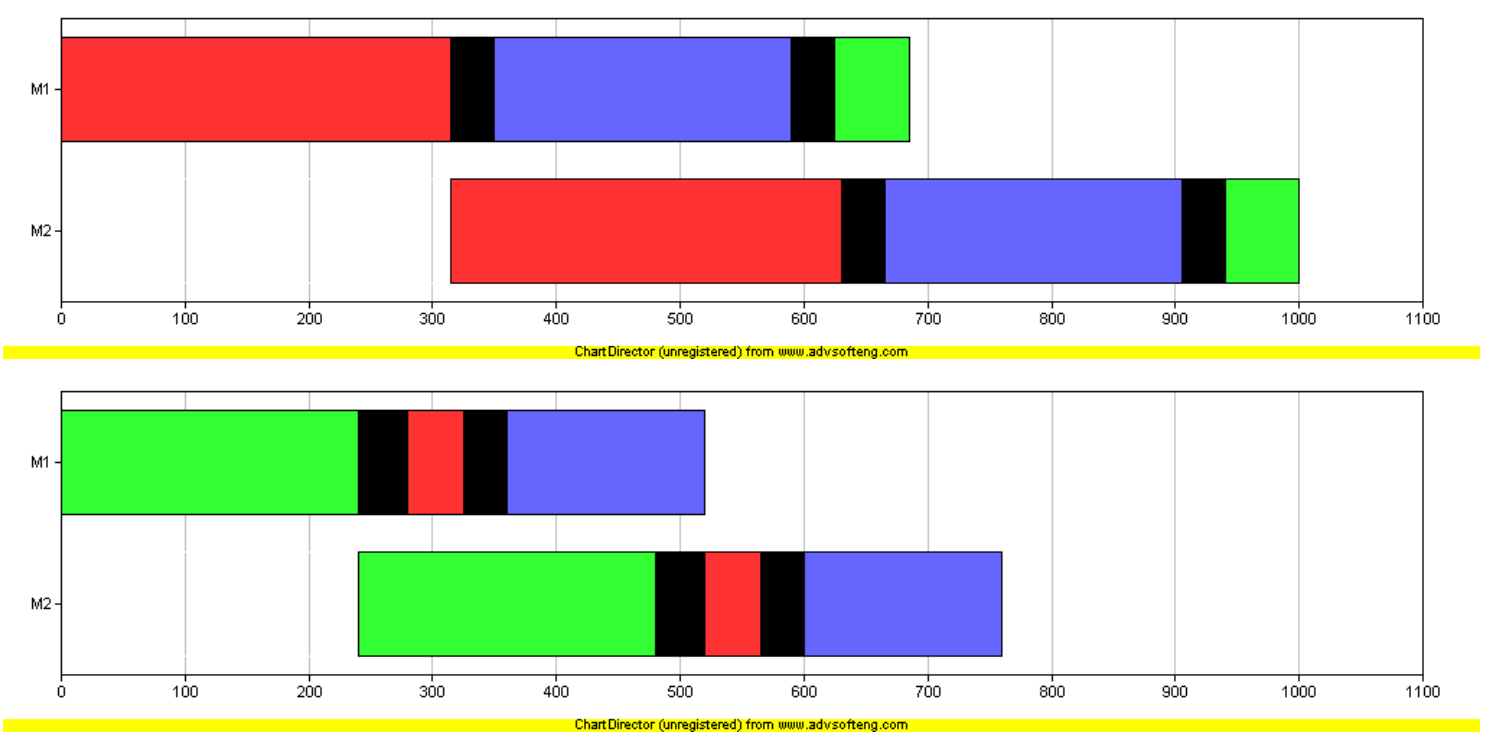

Figura 2: Diagrama de Gantt da primeira solução.
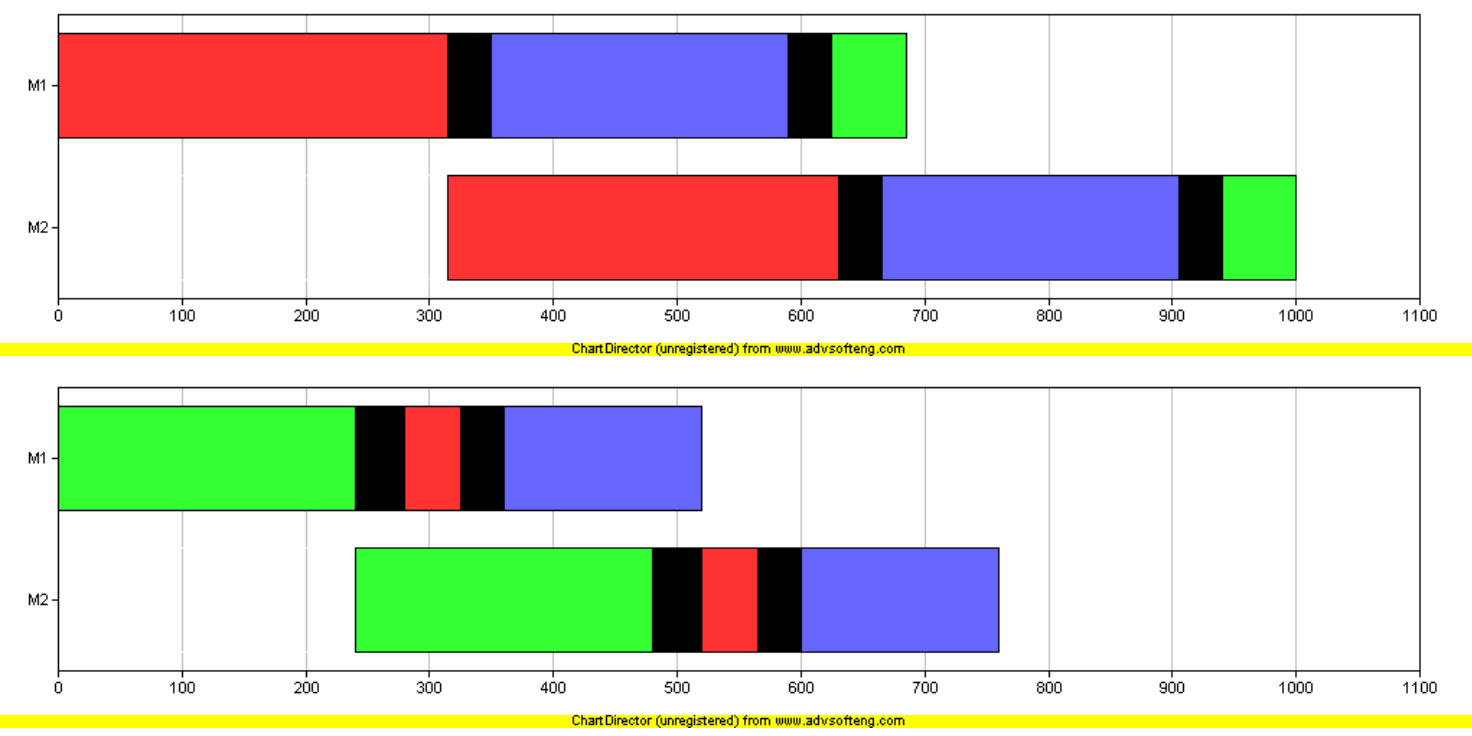

Figura 3: Diagrama de Gantt da segunda solução.

As duas soluções são válidas para as formulações matemáticas $M M O$ e $M M P$, porém, para o modelo matemático $M M R$, apenas a primeira solução é válida, pois a segunda solução é infactível devido a utilização de estoque intermediário (variável $I_{111}=16,67$ ). 
Tabela 8: Primeira solução ótima para o modelo $M M P$.

\begin{tabular}{|lll|}
\hline Sequência & & \\
$y_{111}^{1}=1$ & $y_{131}^{2}=1$ & $y_{321}^{3}=1$ \\
$y_{222}^{1}=1$ & $y_{212}^{2}=1$ & $y_{132}^{3}=1$ \\
Dimensão dos Lotes & & \\
$x_{111}^{1}=175,00$ & $x_{121}^{1}=175,00$ & $x_{311}^{2}=120,00$ \\
$x_{321}^{2}=120,00$ & $x_{211}^{3}=40,00$ & $x_{221}^{3}=40,00$ \\
$x_{212}^{1}=160,00$ & $x_{222}^{1}=160,00$ & $x_{112}^{2}=25,00$ \\
$x_{122}^{2}=25,00$ & $x_{312}^{3}=80,00$ & $x_{322}^{3}=80,00$ \\
Estoque & & \\
$I_{121}=25,00$ & & \\
Produto Fantasma & & \\
$q_{121}^{1}=175,00$ & $q_{222}^{1}=160,00$ & \\
\hline
\end{tabular}

Tabela 9: Segunda solução ótima para o modelo $M M P$.

\begin{tabular}{|lll|}
\hline Sequência & & \\
$y_{111}^{1}=1$ & $y_{131}^{2}=1$ & $y_{321}^{3}=1$ \\
$y_{222}^{1}=1$ & $y_{212}^{2}=1$ & $y_{132}^{3}=1$ \\
Dimensão dos Lotes & & \\
$x_{111}^{1}=183,33$ & $x_{121}^{1}=166,67$ & $x_{311}^{2}=120,00$ \\
$x_{321}^{2}=120,00$ & $x_{211}^{3}=40,00$ & $x_{221}^{3}=40,00$ \\
$x_{212}^{1}=160,00$ & $x_{222}^{1}=160,00$ & $x_{112}^{2}=16,67$ \\
$x_{122}^{2}=33,33$ & $x_{312}^{3}=80,00$ & $x_{322}^{3}=80,00$ \\
Estoque & & \\
$I_{111}=16,67$ & $I_{121}=16,67$ & \\
Produto Fantasma & & \\
$q_{121}^{1}=183,33$ & $q_{222}^{1}=160,00$ & \\
\hline
\end{tabular}




\section{Procedimentos de Solução da Literatura}

A formulação matemática adotada nesta dissertação é o $M M P$, ou seja, um $M I P$ cujas soluções possuem a característica da permutabilidade (modelo matemático (3.15)-(3.28)).

É prática da literatura e da indústria utilizar a mesma sequência de produção em todos os estágios de produção para ambientes flowshop. Dessa forma, no contexto desta dissertação, esta prática será utilizada no desenvolvimento de procedimentos e nas abordagens da literatura. Diversos procedimentos de solução foram implementados, alguns deles estão descritos em Mohammadi et al. (2010a, 2010b) e foram implementados a título de comparação com a metodologia aqui proposta.

Os procedimentos deste capítulo estão divididos em seções distintas. A primeira seção expõe limitantes inferiores conhecidos da literatura. Eles podem indicar se uma solução factível é próxima de uma solução ótima do problema. As próximas seções descrevem heurísticas da literatura baseadas na estratégia de horizonte rolante e outras baseadas na estratégia relax-and-fix.

\subsection{Limitantes Inferiores}

A formulação matemática (3.15)-(3.28) foi, na prática, ineficiente, pois mesmo soluções factíveis para este modelo matemático podem não ser encontradas em tempo computacional razoável pelos softwares comerciais atualmente comercializados. Dessa forma, para promover a comparação entre os métodos, são utilizados limitantes (no caso deste problema, limitantes inferiores). Porém, bons limitantes inferiores (aqueles próximos a uma solução ótima do problema) podem ser difíceis de encontrar.

No trabalho de Mohammadi et al. (2010a) dois limitantes são apresentados para o modelo matemático MMO (3.1)-(3.14). O primeiro limitante inferior (L1) é obtido relaxando todas as variáveis binárias. Uma consequência de relaxar as variáveis binárias é 
que a restrição 3.4, que garante interação vertical, é relaxada, pois o seu impacto sobre o conjunto de soluções pode ser nulo devido às parcelas que contêm o BigM. O segundo limitante inferior (L2) é derivado de $L 1$ e adiciona as expressões 4.1 e 4.2.

$$
\begin{gathered}
\sum_{i=1}^{N} y_{i j m t}^{1}+\sum_{i=1, i \neq j}^{N} \sum_{n=2}^{N} y_{i j m t}^{n}=a_{j m t} \\
j=1, \ldots, N, m=1, \ldots, M, t=1, \ldots, T \\
a_{j m t} \in\{0,1\}
\end{gathered}
$$

A equação 4.1 utiliza a variável binária $a_{j m t}$ para indicar a presença da preparação de máquina em alguma posição da sequência de produção dessa máquina, o que implica que se a máquina $m$, no período $t$ processa o produto $j$, este só pode ser processado em uma única posição da sequência de produção, exceto no caso onde posições adjacentes na sequência de produção pertencem ao mesmo produto $j$ (por isso o $i \neq j$ no segundo somatório). A equação 4.1 só pode ser agregada à relaxação quando os tempos e custos de preparação de máquina obedecem à desigualdade triangular, pois, do contrário, tal equação acrescentaria uma restrição ao modelo, e dessa forma não seria mais um limitante.

Estes limitantes inferiores podem ser adaptados para o modelo matemático $M M P$. Assim, o primeiro limitante inferior (também denominado L1) relaxa todas as variáveis binárias, porém, admitindo apenas soluções permutacionais, a equação equivalente de interação vertical 3.18 deve ser considerada. Para o limitante equivalente a L2, a equação 4.1 é simplificada, pois a sequência de produção das máquinas é a mesma. A equação resultante (4.3) é apresentada abaixo.

$$
\begin{gathered}
\sum_{i=1}^{N} y_{i j t}^{1}+\sum_{i=1, i \neq j}^{N} \sum_{n=2}^{N} y_{i j t}^{n}=a_{j t} \\
j=1, \ldots, N, t=1, \ldots, T \\
a_{j t} \in\{0,1\}
\end{gathered}
$$

No restante desta dissertação, fica empregado os termos L1 e L2 como os limitantes para o modelo matemático $M M P$ ((3.15)-(3.28)). O mesmo exemplo da Seção 3.3, cuja custo de uma solução ótima é de 2360 , possui limitantes inferiores quantificados em 2111, 89 para o L1 e 2359, 86 para L2. 


\subsection{Heurísticas baseadas em Horizonte Rolante}

A estratégia de horizonte rolante para problemas que envolvem decisões de dimensionamento de lotes supõe que decisões de determinados períodos são pouco influentes para alguns períodos mais distantes. Dessa forma, sob uma perspectiva míope, o procedimento heurístico é dividido em iterações inspiradas nos períodos de tempo, iniciando-se nos primeiros períodos e avançando até os últimos. As heurísticas baseadas em horizonte rolante são vistas, de acordo com Mercé e Fontan (2003), como um procedimento iterativo que decompõe o horizonte de planejamento em três seções:

- A seção inicial é composta por períodos cujas decisões já foram tomadas por iterações anteriores e estão parcialmente ou totalmente fixas (congeladas);

- A seção central possui o conjunto de períodos a serem considerados para se tomar decisões;

- A seção final inclue os últimos períodos e que não são considerados para se tomar decisões. Algumas simplificações podem ser utilizadas para tornar o procedimento heurístico mais eficiente.

No início de uma heurística com horizonte rolante, geralmente não há variáveis congeladas, portanto, a seção inicial é vazia. A cada iteração um número de períodos é adicionado à seção inicial, enquanto períodos são subtraídos da seção final, sempre tendo de passar pela seção central. Nesta dissertação são apresentadas três heurísticas baseadas na estratégia de horizonte rolante.

\subsubsection{Primeira Heurística de Horizonte Rolante ( $\mathrm{RH} 1)$}

A heurística RH1 de Mohammadi et al. (2010a) tem como seção central apenas um único período, e toma decisões relativas a este período. Todas as variáveis de decisão dos períodos anteriores (seção inicial) estão fixas. Todas as variáveis inteiras são relaxadas na seção final. A Figura 4 apresenta a divisão do procedimento heurístico em $T$ iterações. Como um conjunto de variáveis são relaxadas, a heurística resolve a cada iteração MIPs menores, tornando o método capaz de obter soluções de maneira mais eficiente. 

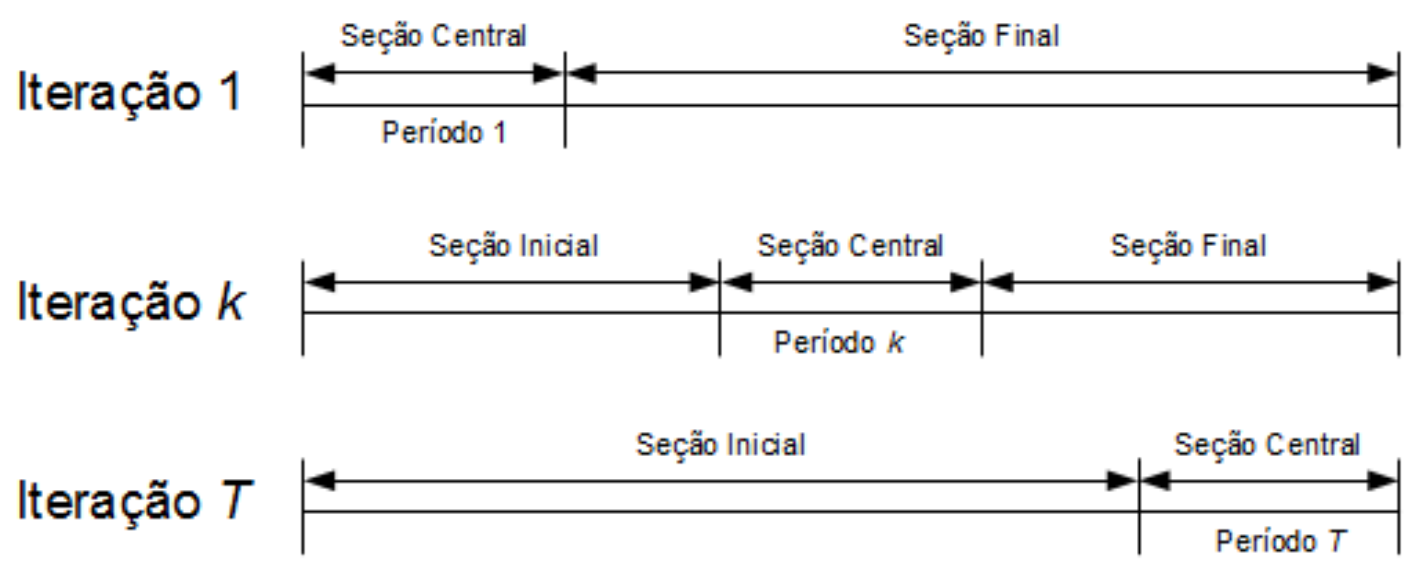

Figura 4: Estratégia de horizonte rolante baseada em Mohammadi et al. (2010a).

\subsubsection{Segunda Heurística de Horizonte Rolante ( $\mathrm{RH} 2)$}

A heurística RH2 de Mohammadi et al. (2010a) é análoga à heurística RH1, exceto pelo fato que as variáveis de decisão fixas na seção inicial são apenas as binárias. Ou seja, apenas as variáveis que definem o sequenciamento nas máquinas são congeladas, enquanto as decisões de dimensionamento de lotes, tamanho do estoque e quantidade de produtos fantasmas estão livres, permitindo que na tomada de decisão de outros períodos essas variáveis assumam valores diferentes.

\subsubsection{Terceira Heurística de Horizonte Rolante $(\mathrm{RH} 3)$}

A heurística H3 de Mohammadi et al. (2010b) é uma adaptação da heurística desenvolvida por Nawaz et al. (1983) (denominada NEH), bastante utilizada na literatura do problema de flowshop, por ser uma heurística simples, flexível e eficiente. A heurística $N E H$ é um procedimento construtivo de inserção onde a cada iteração múltiplas soluções parciais são testadas.

Um passo importante da $N E H$ é a determinação de uma sequência de inserção. A heurística RH3 determina essa ordem da seguinte maneira:

1. Calcula-se o custo total de preparação de máquinas para cada produto distinto $j$ : $W_{j}=\sum_{m=1}^{M} \sum_{i=1}^{N} W_{i j m} ; j=1, \ldots, N$;

2. A sequência de inserção da $N E H$ é feita de acordo com a ordem decrescente de $W_{j}$;

3. Para cada período que a ordem de inserção for feita (por enquanto a sequência de 
inserção independe do período), produtos cuja produção naquele período estão previamente estocados são retirados da ordem de inserção. Assim, quando no período $t$ para o produto $j, I_{j m t-1} \geq d_{j k}$ para todas as máquinas $m$, o produto é retirado da sequência de inserção;

4. Seja $\delta$ a quantidade de produtos retirados da sequência de inserção;

Os próximos passos correspondem à parte de inserção e avaliação da heurística, e, ao final, uma sequência de produção é gerada.

1. Seja a sequência de inserção dada por $\Pi=\left\{\pi_{1}, \pi_{2}, \ldots, \pi_{N-\delta}\right\}$;

2. Para cada iteração $k$, para $k=1, \ldots, N-\delta$ :

- Considere a inserção de $\pi_{k}$ em todas as posições;

- Calcule a soma dos custos de preparação de máquinas resultante;

- Insira $\pi_{k}$ na posição da sequência de produção com menor soma dos custos de preparação de máquina;

3. Como é necessário $N$ posições na sequência de produção, completa-se as posições finais com o último produto produzido;

Assim, as seções da heurística são:

- A seção inicial é composta pelos períodos anteriores à seção central e possui suas variáveis binárias $(y)$ congeladas;

- A seção central é formada apenas pelo período $t$. A partir da sequência de inserção, gera-se a sequência de produção para o período $t$, define as variáveis de sequenciamento $y$ de acordo com a sequência de produção e resolve-se o modelo de programação linear resultante;

- Na seção final as variáveis de decisão inteiras são relaxadas;

\subsection{Heurísticas Relax-and-Fix}

O método relax-and-fix é bem conhecido na literatura, e é bem descrito em Wolsey (1998). O método relaxa todas as variáveis inteiras, e depois, a cada passo do algoritmo, 
determina que um conjunto dessas variáveis sejam novamente inteiras. A partir desse momento resolve-se o MIP com um número menor de variáveis. Fixa-se algumas dessas variáveis de decisão e inicia-se a próxima iteração. O objetivo do método é resolver diversos MIPs menores para alcançar uma solução factível ao final. Para conjuntos de instâncias de tamanho grande o método permite que se encontre soluções mais rapidamente.

\subsubsection{Heurística Relax-and-Fix $(R F)$}

A heurística $R F$ é dividida em $T \cdot N$ iterações, onde a cada uma delas uma variável $y_{i j t}^{n}$ é definida e fixada, ou seja, define-se a cada passo uma posição na sequência de produção de determinado período. A heurística inicia no primeiro período e termina no último período, assim, ao final, as sequências de produção de todos os períodos são definidas e uma solução factível pode ser gerada por meio da resolução de $T \cdot N$ MIPs menores. 


\section{Procedimentos de Solução Propostos}

Neste capítulo os métodos de resolução propostos são apresentados. As abordagens desenvolvidas são baseadas na metaheurística Times Assíncronos, cujos detalhes são vistos no decorrer deste capítulo.

\subsection{Times Assíncronos}

A metaheurística Times Assíncronos (A-Teams) foi proposta por de Souza e Talukdar (1993), porém em Talukdar et al. (1983) já há uma abordagem de solução parecida, proposta também por Talukdar. Um A-Teams é composto por agentes e memórias compartilhadas, e pode ser caracterizado predominantemente por três características:

- Agentes Autônomos: Os agentes tomam suas próprias decisões de seleção de soluções, o momento de uso das mesmas, e controle de alocação de memória;

- Comunicações Assíncronas: Os agentes podem ler e escrever informação nas memórias compartilhadas sem qualquer sincronização entre eles;

- Fluxo de dados cíclico: Os agentes tomam, modificam e salvam informações continuamente nas memórias compartilhadas.

A-Teams são efetivos na resolução de problemas combinatórios de difícil resolução, onde há vários algoritmos implementados, mas que nenhum deles é totalmente satisfatório. Chega-se a um meio termo combinando-os para obter melhores respostas em um tempo reduzido. Exemplos de uso do método são: Talukdar et al. (1983) para problemas não lineares, de Souza (1993) para o problema do caixeiro viajante, Meneses et al. (2008) para problemas de probe selection, dentre outros. Para uma leitura mais avançada no tema, Talukdar et al. (1998) e Talukdar et al. (2003) são boas referências. 
Os agentes são normalmente heurísticas simples, mas metaheurísticas (busca tabu, algoritmo genético, simulated annealing, GRASP, dentre outras) também podem ser usadas. Os agentes são divididos em agentes construtores, destruidores e de melhoria. Os agentes construtores $(A g C)$ são responsáveis pela criação e inclusão de soluções nas memórias compartilhadas. Os agentes de melhoria $(A g M)$ lêem soluções das memórias compartilhadas e, após processamento, retornam soluções para as memórias, que são inseridas juntamente com a eliminação de soluções da memória feita pelos agentes destruidores $(A g D)$. A Figura 5 representa um exemplo da técnica A-Teams com a estrutura de uma única memória compartilhada. Diversos agentes atuam assincronamente nesta memória. Qualquer agente pode ser excluído/incluído do/no conjunto de agentes facilmente. Assim, a heurística é bastante flexível, podendo os agentes ser adicionados ou retirados, inclusive de maneira adaptativa, melhorando o desempenho da heurística.



Figura 5: Exemplo de Arquitetura A-Teams.

\subsection{Times Assíncronos Proposto $(A T)$}

Nesta seção apresentamos a primeira estrutura da metaheurística Times Assíncronos utilizada nesta dissertação. Num primeiro instante são definidos o formato de representação de uma solução e a correspondente estrutura de dados utilizada. Por último, os agentes, classificados como construtores, destruidores e de melhoria, são apresentados. 


\subsubsection{Estrutura de Dados e Formato de Representação}

A estrutura de dados é dividida em três tipos de dados distintos: tarefas, períodos e programações. Uma tarefa é composta de um identificador cujo valor representa o produto correspondente a esta tarefa e dois vetores de tamanho $M$ (número de máquinas), sendo o primeiro vetor representando a dimensão do lote a ser processado em cada máquina e o segundo relativo aos instantes de término dos processos de cada máquina para a tarefa em questão. Os instantes de término são calculados visando diminuir o esforço computacional, já que agentes de melhoria que atuam ao final da sequência de produção não influenciam os instantes de término das tarefas anteriores.

Um período possui duas componentes, uma delas é uma matriz de estoque (de dimensão $N \cdot M)$, relativo ao estoque de cada produto após processamento em cada máquina ao final da programação do período em questão. A segunda componente é um vetor de ponteiros, onde cada posição deste vetor aponta para uma única tarefa. O número de posições deste vetor de ponteiros é $N$, pois por definição do problema matemático, cada período possui uma sequência de operações de $N$ de tarefas. A segunda componente de um período foi assim escolhida para tornar mais rápido computacionalmente a manipulação dos dados pelos agentes da metaheurística.

Por último, uma programação é definida por três componentes, sendo a primeira relativa à função objetivo da programação (valor da solução), a segunda componente representa o somatório da capacidade extra necessária em todas as máquinas para factibilizar a solução, já que, dessa forma, pode-se tratar soluções infactíveis (os detalhes são descritos mais adiante). A componente final é um vetor de dimensão $T$ de períodos. A Figura 6 ilustra a estrutura de dados utilizada. Os valores de alguns dados são relativos à primeira solução da Seção 3.3.

Para facilitar a leitura desta dissertação, representações mais compactas podem ser utilizadas para visualização dos dados de alguma solução do problema. Assim, a Figura 7 representa a primeira solução relativa ao exemplo da Seção 3.3, cujo valor da solução é 2360 unidades monetárias. Os blocos de dados representam períodos, e são subdivididos em tarefas, com a produção por máquina e os instantes de término do processamento de cada máquina. O estoque é apresentado abaixo dos blocos. Para os agentes explicados a seguir alguns dados podem ser suprimidos, como por exemplo os instantes de término, ou até mesmo a representação do tamanho dos lotes.

A memória compartilhada de soluções mantêm uma população de soluções ordenadas 


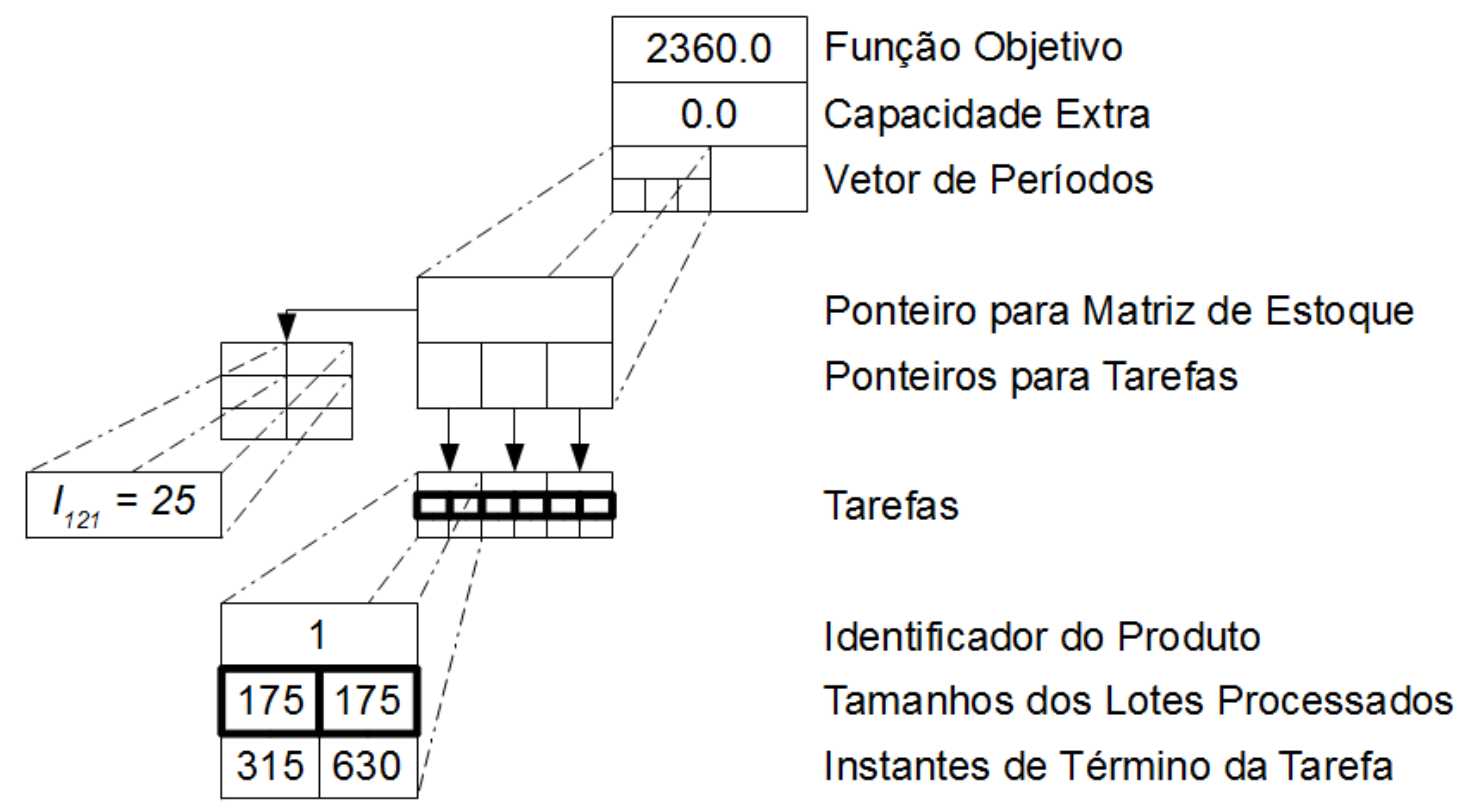

Figura 6: Estrutura de Dados do Problema.

\begin{tabular}{|c|c|c|c|c|c|}
\hline \multicolumn{2}{|c|}{1} & \multicolumn{2}{c|}{3} & \multicolumn{2}{c|}{2} \\
\hline 175 & 175 & 120 & 120 & 40 & 40 \\
\hline 315 & 630 & 590 & 905 & 685 & 1000 \\
\hline \multicolumn{4}{c}{$I_{121}=25$} \\
\end{tabular}

\begin{tabular}{|c|c|c|c|c|c|}
\hline \multicolumn{2}{|c|}{2} & \multicolumn{2}{c|}{1} & \multicolumn{2}{c|}{3} \\
\hline 160 & 160 & 25 & 25 & 80 & 80 \\
\hline 240 & 480 & 325 & 555 & 520 & 760 \\
\hline
\end{tabular}

Figura 7: Representação Compacta de uma Solução para o Problema $M M P$.

de acordo com a sua qualidade. A qualidade de uma solução é medida de acordo com dois critérios: a factibilidade e o valor da função objetivo. Uma solução factível é sempre melhor que uma infactível ou uma solução factível cujo valor da função objetivo é maior que a primeira. Entre soluções infactíveis, aquela que utilizar menos capacidade extra é a melhor. Os agentes atuam sobre a memória compartilhada e são responsáveis pela criação de soluções iniciais, pela melhoria e destruição de soluções. O Algoritmo 1 apresenta o funcionamento da metaheurística Times Assíncronos e de seus agentes. Os agentes AgC, AgM e AgD representam os agentes construtores, de melhoria e destruidores, respectivamente. Considere a memória compartilhada como MEM, e o tamanho máximo desta memória MEMSIZE. No início novas soluções são geradas pelos agentes construtores $(\mathrm{AgC})$ até preencherem toda a memória compartilhada. Em seguida os agentes de melhoria (AgM) atuam nas soluções existentes, buscando por novas soluções (sol1). Enquanto isso, os agentes destruidores (AgD) controlam o número de soluções da memória compartilhada, definindo quais soluções serão inseridas ou retiradas da memória compartilhada. As 
subseções seguintes detalham estes agentes. Para uma visualização resumida dos agentes desenvolvidos vide Tabela 11.

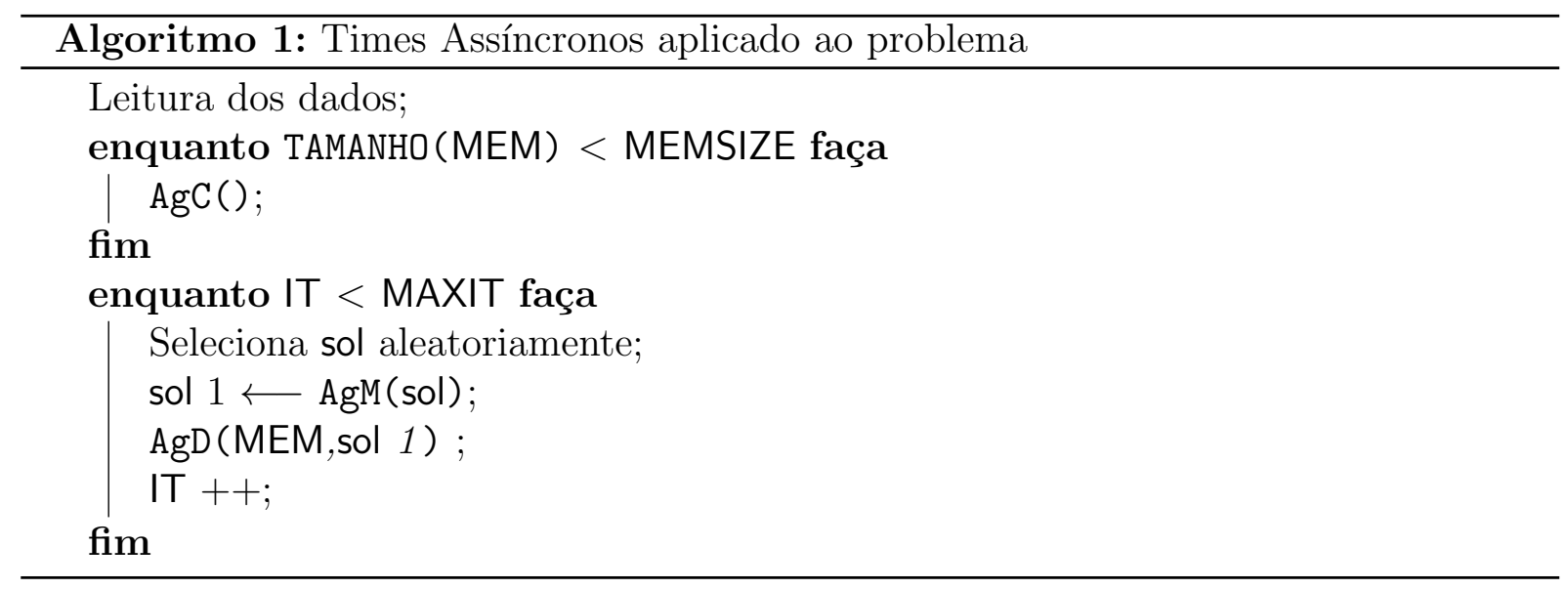

\subsubsection{Agentes Construtores}

Dois agentes construtores foram utilizados pela metaheurística $A T$ e são descritos a seguir:

Agente Construtor $1(\boldsymbol{A g} \boldsymbol{C 1})$ : O primeiro agente construtor dimensiona o número de lotes de cada período, tomando os valores que satisfazem a demanda do mesmo (política lote-por-lote). A sequência dos produtos é dada aleatoriamente. Dessa forma, estoques não são realizados nas soluções geradas. Seja o primeiro período do exemplo da Seção 3.3, com três produtos 1, 2, 3 e cujas demandas são, respectivamente, 150, 40 e 120 unidades. Dessa forma, o agente $A g C 1$ cria uma solução para o período de sequência aleatória, por exemplo uma sequência iniciando com o Produto 3, retornando ao Produto 1 e finalizando com o Produto 2, gerando a sequência de produção $(3,1,2)$. Algumas variáveis dessa sequência de produção são descritas na Tabela 10 e o período é ilustrado na Figura 8.

Tabela 10: Valores positivos do primeiro período do exemplo.

\begin{tabular}{|lll|}
\hline Sequência & & \\
$y_{131}^{1}=1$ & $y_{311}^{2}=1$ & $y_{121}^{3}=1$ \\
Dimensão dos Lotes & & \\
$x_{311}^{1}=120,00$ & $x_{321}^{1}=120,00$ & $x_{111}^{2}=150,00$ \\
$x_{121}^{2}=150,00$ & $x_{211}^{3}=40,00$ & $x_{221}^{3}=40,00$ \\
\hline
\end{tabular}

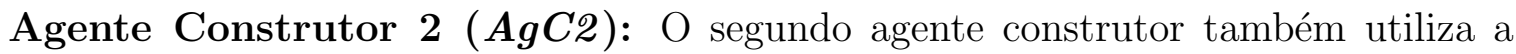
política lote-por-lote para a geração de uma solução inicial. Porém, a sequência de produção de cada período é obtido de maneira análoga à heurística $N E H$, de Nawaz et 


\begin{tabular}{|l|l|l|l|l|l|}
\hline \multicolumn{2}{|c|}{3} & \multicolumn{2}{|c|}{1} & \multicolumn{2}{c|}{2} \\
\hline 120 & 120 & 150 & 150 & 40 & 40 \\
\hline
\end{tabular}

Figura 8: Representação da solução gerada pelo agente construtor $1 \mathrm{AgC1}$.

al. (1983). Alguns detalhes adicionais como a preservação da preparação de máquina e possíveis demandas nulas são considerados. Como descrito anteriormente na heurística $R H 3$, o procedimento heurístico $N E H$ é um procedimento construtivo de inserção, onde a cada iteração múltiplas soluções parciais são testadas. A sequência de inserção utiliza a ordem decrescente do custo total das preparações de máquina para cada produto $j$, $W_{j}=\sum_{m=1}^{M} \sum_{i=1}^{N} W_{i j m} ; j=1, \ldots, N$. São retirados os produtos com demanda nula. O processo construtivo é iniciado inserindo o primeiro produto da sequência de inserção na sequência de produção. O processo prossegue adicionando produto a produto da sequência de inserção na sequência de produção, inserindo de maneira gulosa na melhor posição possível, aquela cujos custos de preparação de máquinas são menores (já que os outros custos são constantes). Observe que ao considerar o custo de preparação de máquinas, a preservação da preparação de máquina também é considerada.

Dado o exemplo numérico da Seção 3.3. O agente $A g C 2$ organiza primeiramente uma sequência de inserção, de acordo com $W_{j}$. No exemplo, $W_{1}=(0+40+70)+(0+40+70)=$ $220, W_{2}=170$ e $W_{3}=170$. Assim, a sequência de inserção é $(1,2,3)$. Seja $C S_{S e q}$, o custo de setup da sequência Seq. Primeiramente inserimos o Produto 1 e obtemos $C S_{(1)}=0$, já que a máquina está previamente preparada para esta tarefa, e só há uma posição de inserção possível. Continuando o processo, inserimos a segunda posição da sequência de inserção, o Produto 2 e obtemos $C S_{(1,2)}=0+50+50=100$ e $C S_{(2,1)}=50+50+40+40=$ 180. Portanto, o produto é inserido na última posição da sequência de produção. Na última inserção, a sequência de produção escolhida é $C S_{(1,3,2)}=140$, que obteve menor custo de preparação, dado que, $C S_{(1,2,3)}=200$ e $C S_{(3,1,2)}=310$. Para o segundo período o raciocínio é análogo, entretanto a máquina inicia o período previamente preparada para o Produto 2. A sequência de produção $(2,1,3)$ é obtida como resultado final para o segundo período. Com o emprego da política lote-por-lote para o dimensionamento de lotes, a solução inicial obtida pelo agente $A g C 2$ para o exemplo numérico é ilustrada na Figura 9 . 


\begin{tabular}{|c|c|c|c|c|c|c|c|c|c|c|c|}
\hline \multicolumn{2}{|c|}{1} & \multicolumn{2}{|c|}{3} & \multicolumn{2}{|c|}{2} & 2 & & \multicolumn{2}{|c|}{1} & \multicolumn{2}{|c|}{3} \\
\hline 150 & 150 & 120 & 120 & 40 & 40 & 160 & 160 & 50 & 50 & 80 & 80 \\
\hline
\end{tabular}

Figura 9: Representação da solução gerada pelo agente construtor 2 ( $A g C 2)$.

\subsubsection{Agentes Destruidores}

Agente de Destruição $(\boldsymbol{A g} \boldsymbol{D})$ : O agente de destruição considera a qualidade da solução. Se a solução gerada por um agente de melhoria é igual a uma das soluções pertencentes à memória compartilhada, então a memória compartilhada fica inalterada. Caso contrário, a solução gerada é inserida na memória compartilhada somente se esta solução é melhor do que a pior solução existente na memória compartilhada. A pior solução da memória compartilhada é removida e apagada.

\subsubsection{Agentes de Melhoria}

Todos os agentes de melhoria escolhem uma solução da memória compartilhada por meio de seleção por torneio de tamanho 2 (Tournament Selection). Assim, são escolhidas, aleatoriamente, duas soluções da memória compartilhada e a melhor delas é utilizada pelo agente de melhoria. Os primeiros agentes focam nas decisões de sequenciamento de tarefas, enquanto os outros agentes visam decisões relacionadas ao dimensionamento dos lotes de produção. Vários agentes de melhoria foram desenvolvidos, e apenas os agentes que obtiveram os melhores resultados estão aqui descritos. Cada agente atua com uma estratégia diferente, buscando por várias maneiras chegar a melhores soluções. Várias configurações destes agentes foram testadas e seus resultados serão apresentados na Seção 6.4 .

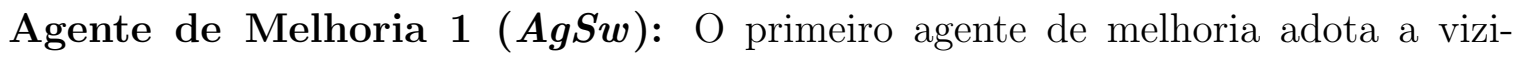
nhança definida por troca de dois produtos quaisquer na sequência de produção (swap). Uma solução inicial é escolhida e o agente analisa certa quantidade de soluções vizinhas, alterando a sequência de produção de um período aleatório, mantendo as decisões relacionadas ao dimensionamento de lotes. O agente retorna a melhor solução encontrada. A Figura 10, parte (a), mostra um exemplo de sequência e de seu vizinho. Neste exemplo, a partir da sequência de produção $(4,2,3,6,5,7,1)$ e após a troca simples entre as posições 2 e 5 , é gerada a sequência $(4,5,3,6,2,7,1)$. Note que para o cálculo dos custos de produção, basta considerar apenas os custos de setup, já que os outros custos são in- 
dependentes para este agente. Para o recálculo da utilização da capacidade da linha de produção, pode-se considerar apenas as posições da sequência de produção a partir das posições trocadas. Assim, no exemplo não é necessário recalcular os instantes de término da primeira tarefa (Posição 1, Produto 4).

Agente de Melhoria 2 ( $\boldsymbol{A g I n}$ ): O segundo agente de melhoria é análogo ao anterior, entretanto a vizinhança definida é de inserção simples, onde se escolhe um item da sequência e o insere em outra posição. Tomando o mesmo exemplo anterior (ilustrado na Figura 10, parte (b)), a sequência de produção $(4,2,3,6,5,7,1)$, e realizando a inserção do produto 2 (que está na posição 2) na posição 5 , a solução gerada possuirá a sequência de produção $(4,3,6,5,2,7,1)$. Os mesmos detalhes quanto ao recálculo da função objetivo e da utilização da capacidade são considerados para aumentar a velocidade do agente.


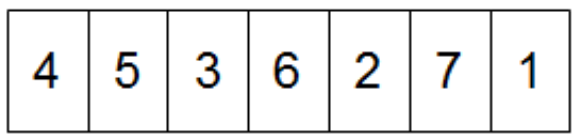

Troca Simples

(a) AgSw

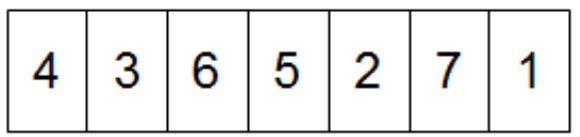

Inserção

(b) Agln

Figura 10: Vizinhanças dos dois primeiros agentes de melhoria.

Agente de Melhoria 3 ( $\boldsymbol{A g B I}$ ): Uma segunda estratégia de inserção é utilizada pelo terceiro agente de melhoria, que busca a melhor posição para inserção de um produto. Esse agente pode ser mais efetivo para problemas-teste com o número de produtos distintos maior. A partir de uma solução inicial, um período e uma tarefa deste período são escolhidos aleatoriamente. A tarefa é inserida em todas as posições possíveis da sequência de produção e a melhor posição (com relação à qualidade da solução) determina a solução final. Considere o exemplo na Figura 11, uma solução inicial com sequência de produção de um período qualquer $(4,2,3,1,5)$. Caso seja escolhida a tarefa na quarta posição (Produto 1), são analisados quatro vizinhos, correspondentes a quatro inserções possíveis que modificam a solução inicial. As soluções estão apresentadas na Figura 11.

Agente de Melhoria $4(\boldsymbol{A g C O})$ : O quarto agente busca melhorar uma solução alterando a sequência de produção de dois períodos consecutivos, alterando o último pro- 

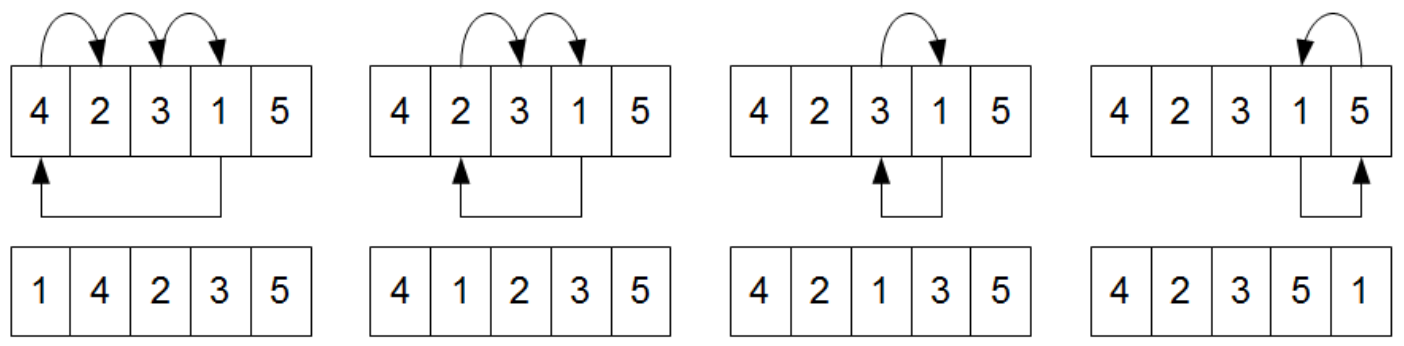

Figura 11: Todas as soluções geradas pelo agente de melhoria $3(A g B I)$.

duto do primeiro dos períodos e a primeira posição da sequência de produção do período seguinte, de tal forma que os produtos que ocupam essas posições sejam os mesmos. As sequências são alteradas utilizando inserção simples. Esse agente aposta que a preservação da preparação das máquinas entre períodos gera melhores soluções. A Figura 12 apresenta um exemplo da ação deste agente. A solução inicial possui no seu período $t$ a sequência de produção $(4,2,3,6,5,7,1)$, e no período $t+1$ possui a sequência $(1,4,7,3,6,2,5)$. Escolhendo o produto 6 para ser posicionado nas sequências de produção dos dois períodos de modo a máquina preservar a preparação de máquina, temos como resultado as sequências $(4,2,3,5,7,1,6)$ e $(6,1,4,7,3,2,5)$, para os dois períodos consecutivos.
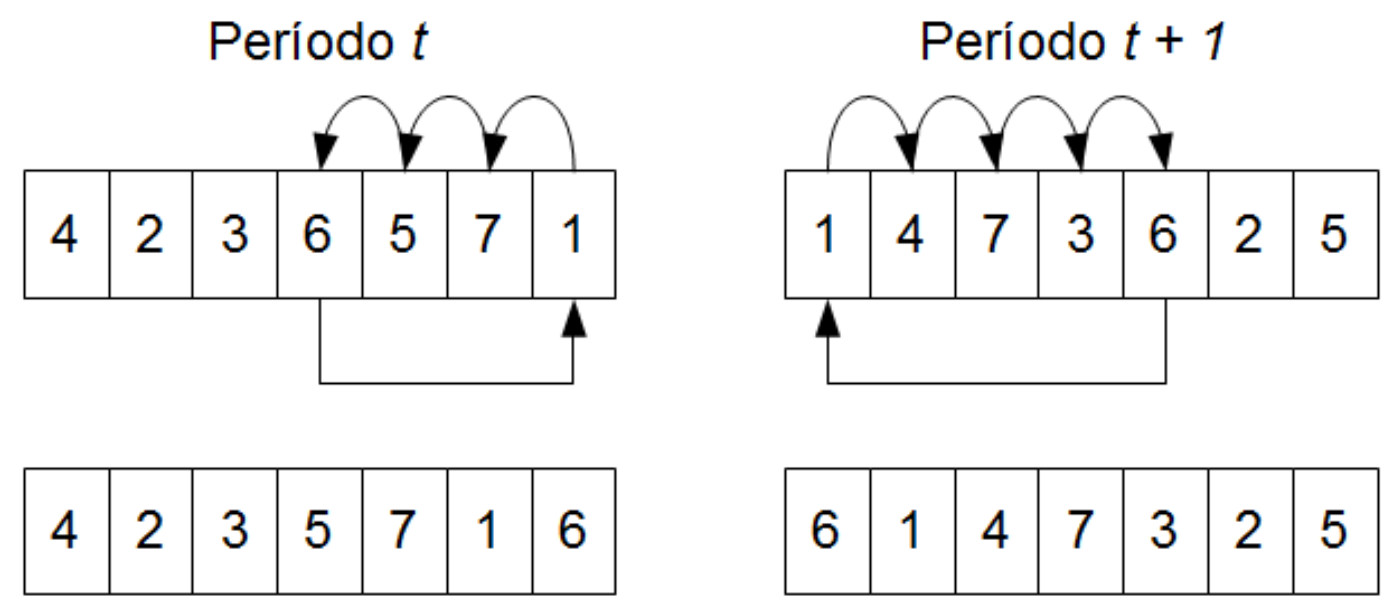

Figura 12: Solução gerada pelo agente de melhoria $4(A g C O)$.

Os agentes de melhoria que tomam decisões de dimensionamento de lotes são apresentados adiante.

Agente de Melhoria $5(\boldsymbol{A g A P})$ : O agente $A g A P$ posterga a produção excedente (com estoque positivo). O agente seleciona aleatoriamente um período com produção excedente de algum produto. Um produto com produção excedente é selecionado tam- 
bém de maneira aleatória e tem toda sua produção excedente postergada para o período imediatamente posterior (período destino). A restrição de atendimento à demanda é respeitada e, por consequência, parte do estoque deste período é eliminada. Caso não haja produção do produto no período destino, a tarefa será inserida numa posição aleatória. A Figura 13 representa um exemplo da atuação do agente $A g A P$, que posterga a produção excedente de 20 unidades de produto acabado do tipo 2.

T1

\begin{tabular}{|c|c|c|c|}
\hline 1 & 3 & \multicolumn{2}{|c|}{2} \\
\hline \begin{tabular}{|l|l|}
150 & 150 \\
\end{tabular} & \begin{tabular}{|l|l|}
120 & 120 \\
\end{tabular} & 60 & 60 \\
\hline & $I_{221}=20$ & & \\
\hline 1 & 3 & & \\
\hline \begin{tabular}{|l|l|}
150 & 150 \\
\end{tabular} & \begin{tabular}{|l|l|}
120 & 120 \\
\end{tabular} & 40 & 40 \\
\hline
\end{tabular}

T2

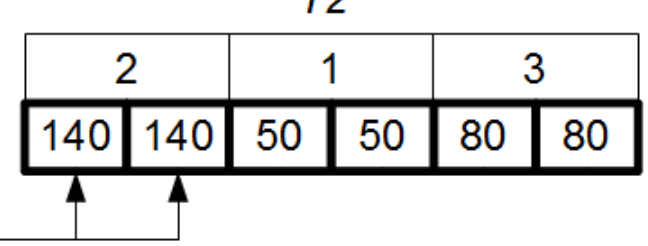

Figura 13: Solução gerada pelo agente de melhoria $5(A g A P)$.

Agente de Melhoria 6 ( $\boldsymbol{A g E P})$ : O segundo agente cuja estratégia foca nas decisões de dimensionamento de lotes adianta a produção de determinados produtos. Este agente analisa a capacidade restante das máquinas para que a solução final gerada não tenha capacidade extra utilizada. O agente $A g E P$ determina dois períodos consecutivos aleatoriamente, tal que o primeiro período possua capacidade disponível para todas máquinas. Aleatoriamente uma tarefa do segundo período é escolhida para ter parte de sua produção adiantada (caso seja possível, a tarefa pode ser completamente adiantada). Caso não haja produção do produto escolhido, determina-se uma posição de maneira aleatória para inserir este produto e adiantar uma parte da produção. Com esse agente, apenas estoques de produtos acabados podem ser gerados. Um exemplo da atuação deste agente está representado na Figura 14. Nela, a partir da solução inicial gerada pelo agente $A g C 2$, chega-se à solução final correspondente à primeira solução ótima no exemplo numérico da Seção 3.3.

Agente de Melhoria 7 (AgEP1): Alguns agentes similares aos segundo agente foram desenvolvidos, cujas estratégias envolvem o adiantamento da produção de determinados períodos. O agente de melhoria $A g E P 1$ foi desenvolvido com a finalidade de buscar soluções que priorizam o adiantamento da produção de produtos intermediários e consequentemente o estoque de produtos inacabados. Esse agente atua adiantando parte da produção de determinada tarefa máquina por máquina. Primeiramente, de maneira gu- 


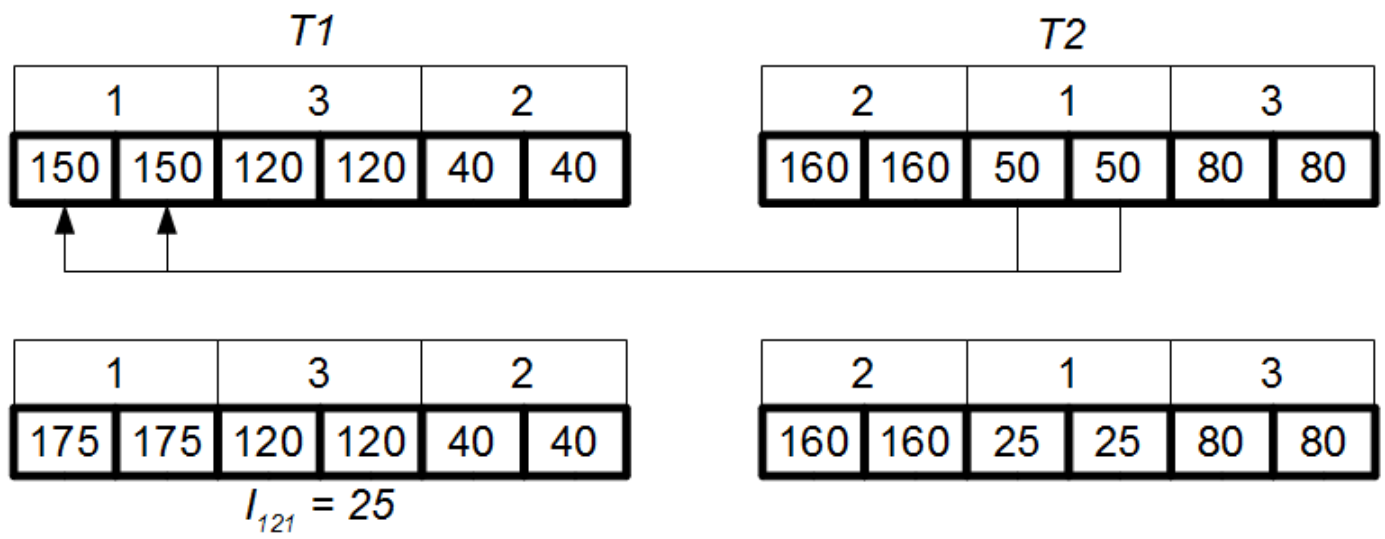

Figura 14: Solução gerada pelo agente de melhoria $6(A g E P)$.

losa, ele visa utilizar a capacidade máxima de produção da primeira máquina, respeitando sempre as capacidades das máquinas posteriores. Esse processo gera estoque intermediário entre as duas primeiras máquinas da linha de produção. O procedimento continua na tentativa de adiantar produção para a segunda máquina, da mesma maneira que feito para a primeira máquina. O processo segue para todas as máquinas, enquanto houver capacidade disponível nas máquinas.

Agente de Melhoria 8 (AgEP2): Outro agente de melhoria análogo ao agente $A g E P$, denominado $A g E P 2$, também busca as tomadas de decisão que adiantam parte da produção de uma tarefa, visando melhores soluções. O procedimento deste agente primeiramente define de maneira aleatória um período com capacidade de produção disponível, um produto produzido no período imediatamente seguinte que terá a sua produção adiantada e uma máquina específica. Esta última escolha especifica a última máquina cuja produção será adiantada, ou seja, o estoque produzido ao final da atuação do agente é após essa máquina. Assim, se a máquina escolhida for a última da linha de produção, a solução final do agente será idêntica a uma solução do agente $A g E P$. Isto torna o agente $A g E P 2$ mais geral que o agente $A g E P$ no sentido de uma maior vizinhança a ser analisada.

Agente de Melhoria 9 ( $\boldsymbol{A g} \boldsymbol{A} \boldsymbol{E P})$ : Uma outra estratégia utilizada é a combinação de agentes que postergam a produção excedente de um determinado produto e adiantam a produção de outro produto. Por exemplo, o agente de melhoria $A g A E P$ considera a união dos agentes $A g A P$ e $A g E P$. Este agente de melhoria é dividido em duas fases. $\mathrm{Na}$ primeira fase posterga-se a produção de um produto excedente do primeiro período escolhido, de maneira análoga ao agente $A g A P$. A segunda fase corresponde a adiantar parte da produção de um produto do segundo período assim como o agente $A g E P$, porém 
o produto deve ser distinto daquele escolhido na fase anterior. Caso a transferência de produção tenha um período destino sem a produção do produto correspodente, inserese este produto em uma posição aleatória. O agente busca possibilidades de melhorias no custo de produção, principalmente pela troca de produtos que estarão em estoque em determinado período, além de possíveis reduções no consumo da capacidade. Como exemplo, a Figura 15 mostra o agente $A g A E P$ atuando sobre uma solução inicial e obtendo ao final a solução ótima do exemplo numérico da Seção 3.3 .

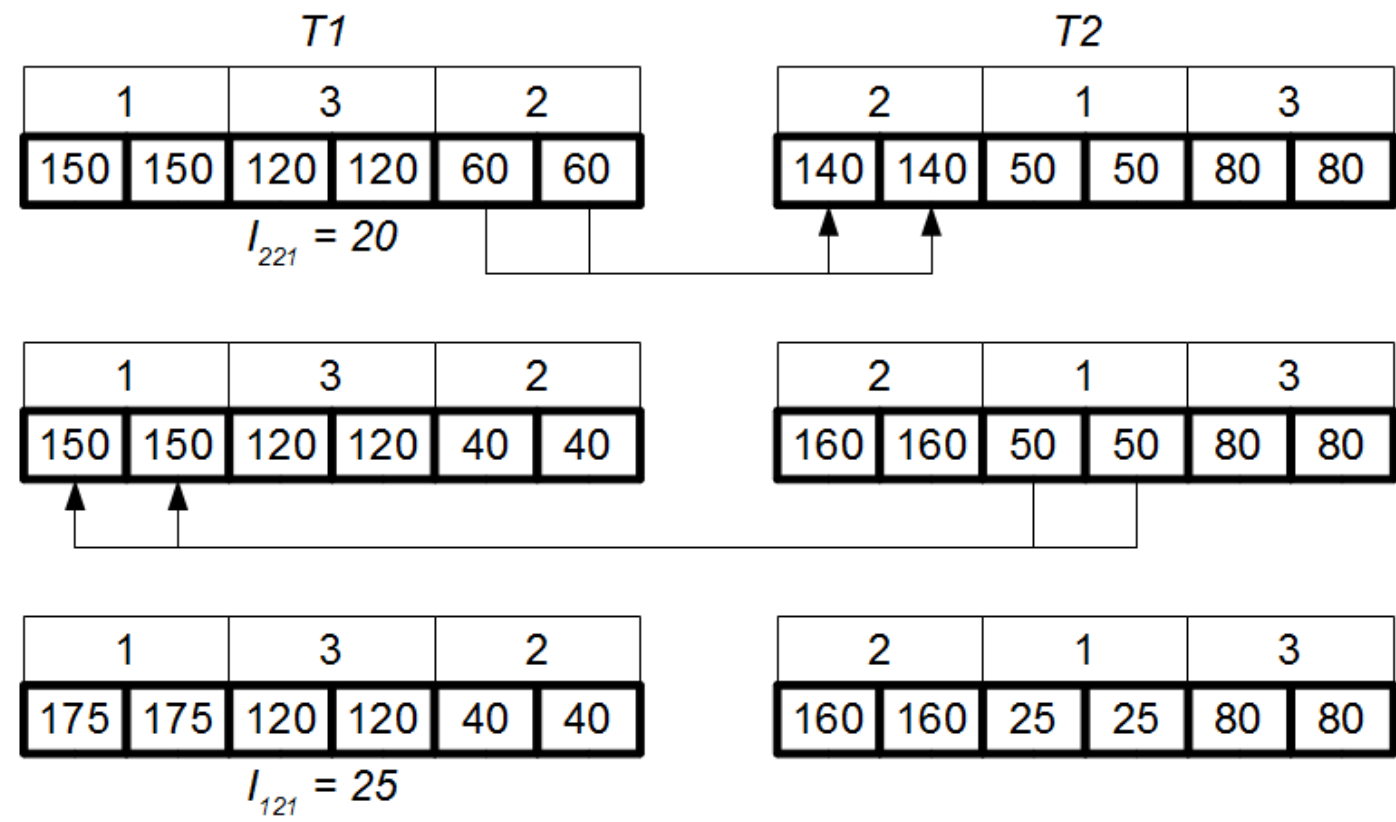

Figura 15: Solução gerada pelo agente de melhoria 9 (AgAEP).

Agente de Melhoria 10 ( $\boldsymbol{A g A E P 1})$ : Da mesma forma que o agente de melhoria $A g A E P$, agentes similares foram gerados a partir da fusão dos agentes $A g A P$ e $A g E P 1$ resultando no agente $A g A E P 1$.

Agente de Melhoria 11 ( $\boldsymbol{A g A E P} 2)$ : Assim como os agentes anteriores, o agente $A g A E P 2$ é resultado da união dos agentes $A g A P$ e $A g E P \mathscr{2}$.

\subsection{Times Assíncronos Híbrido $(\boldsymbol{A T H})$}

Além da metaheurística Times Assíncronos vista anteriormente, propõe-se também a metaheurística Times Assíncronos híbrida. Dessa forma, não apenas procedimentos heurísticos são utilizados, como também são utilizados alguns procedimentos exatos como parte da metaheurística. O uso desses procedimentos pode influenciar a eficiência da 
metaheurística de distintas maneiras. Da maneira como foi proposta, as soluções iniciais proporcionadas pelos novos agentes construtores tendem a ser melhores que do método anterior $(A T)$, já que as decisões de dimensionamento de lotes são tomadas por meio de procedimentos exatos. A estrutura de dados da metaheurística é similar a anterior. Os agentes desenvolvidos são apresentados a seguir. A Tabela 11 apresenta um resumo destes agentes.

\subsubsection{Agentes Construtores}

Agente Construtor Híbrido 1 (HAgC1): O primeiro agente construtor do método $A T H$ tem as sequências de produção para todos os períodos dadas aleatoriamente. Em seguida, começando pelo primeiro período, a primeira tarefa do período é modificada (por inserção simples) para manter a preservação da preparação de máquinas. O mesmo procedimento ocorre para todos os períodos. Fixada a sequência de produção, as decisões relativas ao dimensionamento de lotes são obtidas por meio da resolução exata do modelo matemático de programação linear $L P$ resultante, dado pelas expressões (5.1)-(5.10).

Agente Construtor Híbrido 2 (HAgC2): O segundo agente construtor é similar ao agente construtor ( $H A g C 1)$ no sentido de que as sequências de produção de todos os períodos são geradas e fixadas, e as decisões relativas ao tamanho dos lotes são obtidas por meio da resolução do $L P$ resultante, também dado pelas expressões (5.1)-(5.10). A diferença dos dois agentes é com relação à geração da sequência de produção, que é análoga a do agente construtor $A g C 2$, da metaheurística $A T$. Esse agente determina uma única solução, pois não possui elementos de aleatoriedade nele.

O problema de programação linear resultante de ambos agentes construtores é derivado da formulação matemática $M M P$ (expressões (3.15)-(3.28)). Como as sequências de produção de todos os períodos são fixadas a priori, pode-se reduzir o modelo matemático $M M P$ a um $L P$. Assim, seja a sequência de produção do período $t$ dada por $\Pi_{t}=\pi_{t}^{1}, \pi_{t}^{2}, \ldots, \pi_{t}^{N}$. Pode-se definir $\pi_{t}^{0}$ como o produto no qual a máquina está previamente preparada no período $t$. As variáveis $x_{j m t}^{n}$ e $q_{j m t}^{n}$ são reduzidas às variáveis $x_{m t}^{n} \mathrm{e}$ $q_{m t}^{n}$, já que o índice $j$ é sempre determinado a priori por $\pi_{t}^{n}$.

Para garantir que sempre sejam geradas soluções factíveis, a restrição de capacidade 5.5 foi modificada para admitir horas extras de produção. Isto foi feito adicionando a variável real $e_{m t}$ que representa a capacidade extra utilizada pela máquina $m$ no período t. Da mesma maneira, para coibir a utilização desta capacidade extra, foi adicionado à função objetivo uma parcela que pune esta utilização com um alto custo (BigM). As 
restrições de atendimento da demanda e fluxo de produção (5.2 e 5.3 respectivamente) são válidas para todo produto $j=1, \ldots, N$. Como um mesmo produto pode ser produzido em mais de uma posição, deve-se considerar todas as posições da sequência de produção do período $t, \Pi_{t}$, relativas ao produto $j$, ou seja, deve-se considerar apenas as posições em que $\pi_{t}^{n}=j$. O restante das restrições são análogas ao modelo matemático $M M P$. O LP resultante é apresentado a seguir.

$$
\begin{gathered}
\operatorname{Min} \sum_{n=1}^{N} \sum_{m=1}^{M} \sum_{t=1}^{T} W_{\pi_{t}^{n-1} \pi_{t}^{n} m}+\sum_{n=1}^{N} \sum_{m=1}^{M} \sum_{t=1}^{T} p_{\pi_{t}^{n} m t} \cdot x_{m t}^{n}+ \\
\sum_{j=1}^{N} \sum_{m=1}^{M} \sum_{t=1}^{T} h_{j m} \cdot I_{j m t}+\sum \sum_{m=1}^{M} \sum_{t=1}^{T} B i g M \cdot e_{m t}
\end{gathered}
$$

Sujeito a:

$$
\begin{gathered}
d_{j t}=I_{j M t-1}+\sum_{n=1, \pi_{t}^{n}=j}^{N} x_{M t}^{n}-I_{j M t} \quad j=1, \ldots, N, t=1, \ldots, T \\
I_{j m t-1}+\sum_{n=1, \pi_{t}^{n}=j}^{N} x_{m t}^{n}=I_{j m t}+\sum_{n=1, \pi_{t}^{n}=j}^{N} x_{m+1 t}^{n} \\
j=1, \ldots, N, m=1, \ldots, M-1, t=1, \ldots, T \\
\sum_{n=1}^{n^{\prime}} S_{\pi_{t}^{n-1} \pi_{t}^{n} m}+\sum_{n=1}^{n^{\prime}} b_{\pi_{t}^{n} m} \cdot q_{m t}^{n}+\sum_{n=1}^{n^{\prime}} b_{\pi_{t}^{n} m} \cdot x_{m t}^{n} \leq \\
\sum_{n=1}^{n^{\prime}} S_{\pi_{t}^{n-1} \pi_{t}^{n} m+1}+\sum_{n=1}^{n^{\prime}} b_{\pi_{t}^{n} m+1} \cdot q_{m+1 t}^{n}+\sum_{n=1}^{n^{\prime}-1} b_{\pi_{t}^{n} m+1} \cdot x_{m+1 t}^{n} \\
n^{\prime}=1, \ldots, N, m=1, \ldots, M-1, t=1, \ldots, T \\
\sum_{n=1}^{N} S_{\pi_{t}^{n-1} \pi_{t}^{n} m}+\sum_{n=1}^{N} b_{\pi_{t}^{n} m} \cdot q_{m t}^{n}+\sum_{n=1}^{N} b_{\pi_{t}^{n} m} \cdot x_{m t}^{n} \leq C_{m t}+e_{m t} \\
m=1, \ldots, M, t=1, \ldots, T \\
x_{m t}^{n} \leq\left(\frac{C_{m t}}{b_{\pi_{t}^{n} m}}\right) \\
n=1, \ldots, N, m=1, \ldots, M, t=1, \ldots, T, \pi_{t}^{n-1}=\pi_{t}^{n} \\
q_{m t}^{n} \leq\left(\frac{C_{m t}}{b_{\pi_{t}^{n} m}}\right) \\
n=1, \ldots, N, m=1, \ldots, T, \pi_{t}^{n-1} \neq \pi_{t}^{n} \\
n=1, t=1, \ldots \\
n=1, \ldots
\end{gathered}
$$




$$
\begin{gathered}
n=1, \ldots, N, m=1, \ldots, M, t=1, \ldots, T \\
I_{j m t}, x_{m t}^{n}, q_{m t}^{n}, e_{m t} \geq 0 \\
I_{j m 0}=0 \quad j=1, \ldots, N, m=1, \ldots, M
\end{gathered}
$$

\subsubsection{Agentes Destruidores}

O agente de destruição é análogo ao agente da metaheurística $A T$ e considera a qualidade da solução.

\subsubsection{Agentes de Melhoria}

Os agentes de melhoria são os mesmos da metaheurística AT. São eles os agentes de melhoria $A g S w, A g I n, A g B I, A g C O$ e os agentes de melhoria que focam na tomada de decisões relacionadas ao dimensionamento de lotes, os agentes $A g E P, A g A P$ e $A g A E P$. Segue a Tabela 11, que apresenta de forma resumida as funções de cada agente desenvolvido para as metaheurísticas $A T$ e $A T H$.

Tabela 11: Resumo dos agentes desenvolvidos.

\begin{tabular}{|c|l|}
\hline Agentes & Descrição \\
\hline Agentes Construtores & \\
\hline$A g C 1$ & Sequência de produção aleatória, política lote-por-lote \\
$A g C 2$ & Sequência de produção obtida como a heurística $N E H$, política lote-por-lote \\
$H A g C 1$ & Sequência de produção aleatória, dimensionamento de lotes por $L P$ \\
SAgC2 & \\
\hline Agentes Destruidores & \\
\hline$A g D$ & Substitui a pior solução da memória compartilhada com relação a qualidade \\
\hline Agentes de Melhoria & \\
\hline$A g S w$ & Busca sequências de produção melhores na vizinhança de troca simples \\
$A g I n$ & Busca sequências de produção melhores na vizinhança de inserção simples \\
$A g B I$ & Busca sequências de produção melhores na vizinhança de inserção simples, para todas posições possíveis \\
$A g C O$ & Busca sequências de produção melhores para períodos consecutivos, promovendo a preservação do setup \\
$A g A P$ & Posterga produção excedente de um produto \\
$A g E P$ & Adianta a produção, visa estoque de produtos acabados \\
$A g E P 1$ & Adianta a produção de maneira gulosa, máquina a máquina \\
$A g E P 2$ & Adianta a produção, visa estoque de produtos intermediários em uma máquina aleatória \\
$A g A E P$ & Fusão dos agentes $A g A P$ e $A g E P$ \\
$A g A E P 1$ & Fusão dos agentes $A g A P$ e $A g E P 1$ \\
$A g A E P 2$ & Fusão dos agentes $A g A P$ e $A g E P 2$ \\
\hline
\end{tabular}




\section{Experimentos Computacionais}

\subsection{Ambiente de Teste}

O algoritmo usado para a geração de números aleatórios é uma implementação do multiplicative linear congruential generator, com parâmetros 16807 (multiplicador) e $2^{31}-1$ (número primo). Este algoritmo é descrito em (PARK; MILLER, 1988). Todos os testes foram feitos usando um Intel Core 2 Duo E7500 com velocidade de 2.93G $\mathrm{Hz}$, memória L2 cache de $3 M B$ e $3,2 G B$ de RAM sob o sistema operacional Windows 7 . Todos os algoritmos foram implementados em $\mathrm{C} / \mathrm{C}++$. Para resolução dos problemas de programação linear e programação linear inteira mista, o software IBM ILOG CPLEX, na versão 12.1 foi utilizado, por meio da biblioteca de classes e funções CPLEX Concert Technology.

\subsection{Problemas-Teste Gerados}

Os problemas-teste foram gerados de acordo com os experimentos propostos no artigo de (MOHAMmadi et al., 2010a). Considerando as combinações do número de produtos, máquinas e períodos como $(N, M, T)$, os problemas-teste possuem as configurações distintas consideradas: $(3,3,3),(5,3,3),(3,5,3),(3,3,5),(5,5,5),(7,5,5),(5,7,5)$, $(5,5,7),(7,7,7),(10,5,5),(5,10,5),(5,5,10),(10,7,7),(7,10,7),(7,7,10),(10,10,10)$, $(15,10,10),(10,15,10),(10,10,15)$ e $(15,15,15)$.

Os parâmetros de produção são gerados de maneira uniforme, utilizando a função aleatória descrita por (PARK; MILLER, 1988), de acordo com intervalos e valores propostos em (MOHAMMADI et al., 2010a). Dessa forma, definido U(min; max; passo) como a escolha aleatória no intervalo fechado entre min e max, dado o passo, que restringe o número de elementos disponíveis no intervalo ao conjunto $\{\min ; \min +$ passo $; \ldots ; \max -$ passo; $\max \}$ e considerando que o max - min é divisível pelo passo, os parâmetros são gerados como a seguir: 
- Demanda $d_{j t}: U(0 ; 180 ; 10)$

- Custos de estoque $h_{j m}: U(0,2 ; 0,4 ; 0,1)$

- Tempos de processamento $b_{j m}: U(1,5 ; 2,0 ; 0,1)$

- Custos de processamento $p_{j m t}: U(1,5 ; 2,0 ; 0,1)$

- Tempos de setup $S_{i j m}: U(35 ; 70 ; 5)$

- Custos de setup $W_{i j m}: U(35 ; 70 ; 5)$

Ao início da programação de produção todas as máquinas estarão configuradas para determinado produto $\left(j_{0 m}=U(1, N, 1), m \in\{1, \ldots, M\}\right)$. O último parâmetro, a capacidade das máquinas, é gerado de acordo com a capacidade mínima necessária no caso crítico (onde todos os parâmetros assumem os valores máximos). Assim, considerando uma linha de produção de $\mathrm{N}$ produtos, $\mathrm{M}$ máquinas e $\mathrm{T}$ períodos, o caso crítico teria demanda máxima $d_{j t}=180 ; j=1, \ldots, N, t=1, \ldots, T$, tempos de processamento $b_{j m}=2,0 ; j=1, \ldots, N, m=1, \ldots, M$ e tempos de preparação de máquina $S_{i j m}=70 ; j, i=1, \ldots, N, m=1, \ldots, M$. Dessa forma o cálculo da capacidade necessária para esse caso é dado de acordo com a Figura 16.

Na Figura 16, $m_{m}$ representam as máquinas, $j_{j}$ as tarefas (a ordem das tarefas é independente, já que os tempos de processamento são da mesma magnitude) e $C_{m t}$ representa a capacidade mínima necessária para o caso crítico em um período qualquer $t$. Alguns instantes são especificados para a compreensão:

- O intervalo $\left(0, t_{1}\right)$ representa o tempo de preparação de máquina da primeira tarefa (dado que o produto desta tarefa não é o mesmo que a máquina já está preparada) e $t_{1}=70$



Figura 16: Diagrama de Gantt apresentando a capacidade mínima. 
- O intervalo $\left(t_{1}, t_{3}\right)$ denota o tempo de processamento da tarefa $j_{1}$ na máquina $m_{1}$, assim, $t_{3}-t_{1}=2 \cdot 180=360$ e $t_{3}=430 ;$

- O instante $t_{2}$ é o início do setup na máquina $m_{2}$. As preparações de máquina são antecipatórias;

- $t_{4}$ é o instante de término da tarefa $j_{1}$ na máquina $m_{2}, t_{4}=70+360+360=$ $70+2 * 360=790$

- Adiante, $t_{6}$ é o instante de término da tarefa $j_{1}$ na máquina $m_{M-1}, t_{6}=70+(M-$ 1) *360;

- Analogamente, $t_{7}=70+M * 360$;

- $t_{8}$ é o instante de término da tarefa $j_{2}$ na máquina $m_{M}, t_{8}=t_{7}+70+360=$ $(M-1) * 360+2 * 70+2 * 360$;

- $t_{10}$ é o instante de término da tarefa $j_{N-1}$ na máquina $m_{M}, t_{10}=(M-1) * 360+$ $(N-1) * 70+(N-1) * 360$;

- $t_{11}$ é o instante de término da tarefa $j_{N}$ na máquina $m_{M}, t_{11}=(M-1) * 360+N *$ $70+N * 360$

- Assim, a capacidade mínima da máquina $m_{M}, C_{M t}$, equivale a $(M-1) * 360+$ $N * 70+N * 360$. Pode-se inferir, dessa maneira, que para a máquina $m_{m}$, sua capacidade mínima requerida pelo caso crítico é de

$$
C_{m t}=(m-1) * 360+N * 70+N * 360=(m-1) * 360+N * 430
$$

Para a combinação $(5,3,3)$, no caso crítico, as capacidades mínimas das máquinas seriam: $C_{1 t}=2150, C_{2 t}=2510$ e $C_{3 t}=2870$. Foram realizados testes que consideram desde $60 \%$ dessas capacidades, até $80 \%$ das mesmas, com o passo de $10 \%$, visando analisar a robustez do algoritmo, tanto para casos com capacidades reduzidas quanto casos com capacidades maiores. Vale ressaltar que, para alguns problemas-teste, menores capacidades tornam o exemplar infactível.

Para cada classe de problemas-teste, representada por sua combinação $(N, M, T)$, cinco exemplares incompletos (sem os dados das capacidades das máquinas) foram gerados. Como são três distintos valores para as capacidades das máquinas, cada problemateste incompleto resultou em três exemplares completos, portanto cada classe possui um total de quinze problemas-teste. Como são vinte classes, variando de $(3,3,3)$ a $(15,15,15)$, 
um total de trezentos problemas-teste foram gerados e utilizados para testar todos os procedimentos desenvolvidos.

\subsection{Parâmetros dos Procedimentos}

Para todos os procedimentos foi imposto um tempo máximo de resolução de 7200 segundos (2 horas). As heurísticas da literatura são divididas em iterações em que cada uma delas resolve um problema de programação inteira mista de tamanho reduzido comparado ao tamanho original. Para essas heurísticas, o tempo máximo de resolução é igualmente dividido para cada iteração. Assim, o procedimento heurístico $R F$, baseado na estratégia relax-and-fix, cujo número total de iterações é dado por $N \cdot T$ (número de posições da sequência de produção em um período pelo número de períodos), possui para cada iteração um tempo de $\frac{7200}{N \cdot T}$ segundos. Por exemplo, para um problema-teste da classe $(10,5,5)$, onde $R F$ utiliza 50 iterações, cada iteração possui tempo de 144 segundos. A cada iteração, caso uma solução factível tenha sido encontrada, o procedimento continua, caso contrário, o procedimento é finalizado.

As metaheurísticas desenvolvidas para o problema (do tipo AT e $A T H$ ) possuem uma única memória compartilhada com capacidade de vinte soluções. Testes também foram feitos com capacidade da memória compartilhada de dez soluções para a metaheurística $A T H$. A precisão utilizada nos procedimentos heurísticos foi fixada em $10^{-4}$, ou seja, qualquer número real menor (em módulo) que $10^{-4}$ é considerado zero. Uma iteração para as metaheurísticas foi definida como o processo de cálculo da função objetivo e da capacidade extra utilizada, dada uma programação de produção (solução). Assim, agentes podem consumir uma ou mais iterações. Para determinados agentes foi instituído que o número máximo de soluções a serem consideradas em uma vizinhança de busca é dez soluções vizinhas. O número de iterações das metaheurísticas foi fixado em cem mil. Todos os testes computacionais de metaheurísticas, por possuirem elementos aleatórios, foram simulados cinco vezes.

\subsection{Resultados Computacionais}

Os resultados completos de todos os problemas-teste são apresentados nos Apêndices C e D, respectivamente os valores das soluções encontradas e os tempos computacionais consumidos. Nesta seção os resultados são apresentados e as principais características re- 
sultantes são descritas adiante. Para tanto, a seção foi subdividida em algumas subseções de resultados: Procedimentos da Literatura, Metaheurísticas Propostas e Resultados Finais.

\subsubsection{Procedimentos da Literatura}

As abordagens da literatura são compostas de procedimentos de resolução exata, limitantes inferiores e alguns procedimentos heurísticos. A resolução do modelo matemático de maneira exata geralmente consome muito tempo computacional, sendo assim, resolvese de maneira ótima apenas algumas poucas classes de problemas-teste. A partir de problemas-teste da classe $(5,5,5)$ os resultados computacionais demonstraram que já não há garantias de que uma solução ótima seja encontrada pelo método exato. Com relação aos limitantes inferiores, no tempo computacional máximo de 7200 segundos, é viável obter o limitante $L 1$ para a maioria dos problemas-teste.

Na Tabela 12 é apresentada uma comparação entre os limitantes e os valores de soluções ótimas para as classes de problemas-teste. Os valores representam a média da soluções encontradas. A coluna MIP considera todas as soluções do procedimento exato, enquanto a coluna $M I P^{*}$ somente aquelas que são comprovadamente ótimas. As colunas seguintes apresentam a média das soluções dos limitantes inferiores $L 1$ e L2 e as últimas colunas apresentam a média da diferença relativa ( $g a p)$ entre essas soluções. O símbolo "-" é utilizado quando não ocorre nenhuma comparação entre as abordagens, ou quando nenhuma solução de determinada classe foi encontrada.

Com relação a Tabela 12, pode-se inferir que o segundo limitante inferior (L2) é notadamente superior ao limitante $L 1$, porém para muitos problemas-teste não foi possível obter L2 no tempo máximo permitido (lembre-se que o limitante L2 considera variáveis binárias). Para alguns problemas-teste a solução do limitante L2 é uma solução ótima do problema original.

Além disso, a partir da Tabela 12, podemos deduzir uma classificação para as classes relacionando os resultados ao tamanho das classes. As classes de problemas-teste de pequeno porte são aquelas cujas soluções ótimas foram sempre encontradas pela resolução do MIP correspondente. As classes classificadas como grande porte são aquelas que nem mesmo uma solução factível foi encontrada pela resolução do MIP. Assim, os problemasteste de pequeno porte são aqueles das classes $(3,3,3),(5,3,3),(3,5,3)$ e $(3,3,5)$ enquanto os problemas-teste de grande porte pertencem às classes $(10,7,7),(10,10,10),(15,10,10)$, $(10,15,10),(10,10,15)$ e $(15,15,15)$. O restante dos problemas-teste pertencem a classes 
Tabela 12: Comparação entre os limitantes inferiores e a solução exata.

\begin{tabular}{|c|c|c|c|c|c|c|c|c|c|}
\hline & MIP & $M I P^{*}$ & L1 & $L 2$ & $L 1 / M I P^{*}$ & $L 2 / M I P^{*}$ & $L 1 / M I P$ & $L 2 / M I P$ & $L 1 / L 2$ \\
\hline$(3,3,3)$ & 5379,03 & 5379,03 & 4812,51 & 5327,10 & $10,86 \%$ & $1,42 \%$ & $10,86 \%$ & $1,42 \%$ & $9,69 \%$ \\
\hline$(5,3,3)$ & 7880,49 & 7880,49 & 6920,98 & 7783,99 & $12,45 \%$ & $1,62 \%$ & $12,45 \%$ & $1,62 \%$ & $11,13 \%$ \\
\hline$(3,5,3)$ & 6654,18 & 6654,18 & 6158,35 & 6897,65 & $11,86 \%$ & $1,10 \%$ & $11,86 \%$ & $1,10 \%$ & $10,82 \%$ \\
\hline$(3,3,5)$ & 7582,30 & 7582,30 & 6948,29 & 7670,00 & $9,87 \%$ & $0,31 \%$ & $9,87 \%$ & $0,31 \%$ & $9,56 \%$ \\
\hline$(5,5,5)$ & 22468,82 & 21726,89 & 20484,24 & 22329,28 & $10,55 \%$ & $1,20 \%$ & $11,25 \%$ & $1,87 \%$ & $9,57 \%$ \\
\hline$(7,5,5)$ & 30164,79 & - & 26478,75 & 29386,02 & - & - & $12,20 \%$ & $2,56 \%$ & $9,90 \%$ \\
\hline$(5,7,5)$ & 30353,46 & 22853,62 & 27539,68 & 30556,66 & $11,78 \%$ & $1,62 \%$ & $11,79 \%$ & $2,69 \%$ & $9,91 \%$ \\
\hline$(5,5,7)$ & 30563,22 & - & 27313,94 & 29796,01 & - & - & $10,62 \%$ & $2,49 \%$ & $8,33 \%$ \\
\hline$(7,7,7)$ & 58605,56 & - & 51627,12 & 57531,28 & - & - & $13,81 \%$ & $3,17 \%$ & $8,81 \%$ \\
\hline$(10,5,5)$ & 49626,11 & - & 41471,23 & 46578,87 & - & - & $16,47 \%$ & $6,55 \%$ & $10,39 \%$ \\
\hline$(5,10,5)$ & 41033,56 & 33169,87 & 37428,99 & 41331,12 & $12,04 \%$ & $2,10 \%$ & $12,37 \%$ & $3,35 \%$ & $9,52 \%$ \\
\hline$(5,5,10)$ & 44465,56 & - & 39691,09 & 42673,26 & - & - & $10,71 \%$ & $2,84 \%$ & $7,84 \%$ \\
\hline$(10,7,7)$ & - & - & 75770,10 & - & - & - & - & - & - \\
\hline$(7,10,7)$ & 86716,43 & - & 75805,19 & - & - & - & $17,47 \%$ & - & - \\
\hline$(7,7,10)$ & 91582,87 & - & 77710,68 & - & - & - & $14,18 \%$ & - & - \\
\hline$(10,10,10)$ & - & - & 160720,20 & - & - & - & - & - & - \\
\hline$(15,10,10)$ & - & - & 232200,17 & - & - & - & - & - & - \\
\hline$(10,15,10)$ & - & - & 242476,20 & - & - & - & - & - & - \\
\hline$(10,10,15)$ & - & - & 240603,78 & - & - & - & - & - & - \\
\hline$(15,15,15)$ & - & - & 523849,45 & - & $\begin{array}{lll}- & & \end{array}$ & - & - & - & - \\
\hline
\end{tabular}

Tabela 13: Média dos tempos computacionais por classe de problema-teste.

\begin{tabular}{|c|ccccccc|}
\hline & $M I P$ & $L 1$ & $L 2$ & $R H 1$ & $R H 2$ & $R H 3$ & $R F$ \\
\hline$(3,3,3)$ & 0,43 & 0,04 & 0,15 & 0,44 & 0,50 & 0,09 & 0,60 \\
$(5,3,3)$ & 17,07 & 0,07 & 0,63 & 1,94 & 2,17 & 0,14 & 1,79 \\
$(3,5,3)$ & 0,51 & 0,04 & 0,26 & 0,67 & 0,65 & 0,10 & 0,72 \\
$(3,3,5)$ & 0,90 & 0,04 & 0,40 & 0,71 & 0,87 & 0,15 & 1,25 \\
$(5,5,5)$ & 4878,90 & 0,24 & 26,73 & 16,15 & 20,58 & 0,52 & 6,23 \\
$(7,5,5)$ & 7200,08 & 0,73 & 274,22 & 357,43 & 356,12 & 1,19 & 25,59 \\
$(5,7,5)$ & 5772,33 & 0,46 & 42,36 & 45,08 & 46,94 & 0,72 & 9,79 \\
$(5,5,7)$ & 7200,06 & 0,41 & 250,97 & 35,93 & 39,80 & 1,04 & 13,18 \\
$(7,7,7)$ & 7200,12 & 2,77 & 4702,02 & 1844,37 & 2062,68 & 5,06 & 116,47 \\
$(10,5,5)$ & 7200,14 & 2,58 & 3834,31 & 5028,66 & 4043,58 & 3,39 & 143,89 \\
$(5,10,5)$ & 6538,41 & 0,90 & 97,56 & 147,78 & 159,12 & 1,13 & 18,02 \\
$(5,5,10)$ & 7200,07 & 0,64 & 3664,74 & 70,43 & 79,83 & 2,17 & 25,51 \\
$(10,7,7)$ & - & 12,55 & - & 5975,86 & 5382,82 & 13,83 & 971,11 \\
$(7,10,7)$ & 7200,16 & 7,01 & - & 3856,73 & 4069,53 & 10,08 & 303,33 \\
$(7,7,10)$ & 7200,07 & 4,79 & - & 3590,78 & 3300,00 & 11,13 & 333,43 \\
$(10,10,10)$ & - & 77,60 & - & 6487,27 & - & 145,01 & 4427,21 \\
$(15,10,10)$ & - & 445,71 & - & - & - & 1097,77 & - \\
$(10,15,10)$ & - & 316,93 & - & - & - & 502,90 & - \\
$(10,10,15)$ & - & 198,28 & - & - & - & 608,59 & - \\
$(15,15,15)$ & - & 3484,73 & - & - & - & 6548,57 & - \\
\hline
\end{tabular}

Tabela 14: Número de problemas-teste não resolvidos por classe.

\begin{tabular}{|c|ccccccc|c|}
\hline & $M I P$ & $L 1$ & L2 & RH1 & RH2 & RH3 & RF & Inf \\
\hline$(3,3,3)$ & 1 & 0 & 0 & 4 & 1 & 2 & 2 & 1 \\
$(5,3,3)$ & 1 & 0 & 0 & 1 & 1 & 1 & 1 & 1 \\
$(3,5,3)$ & 4 & 1 & 1 & 4 & 4 & 4 & 4 & 4 \\
$(3,3,5)$ & 3 & 0 & 0 & 4 & 3 & 3 & 3 & 3 \\
$(5,5,5)$ & 3 & 0 & 1 & 4 & 3 & 4 & 4 & 2 \\
$(7,5,5)$ & 0 & 0 & 0 & 0 & 0 & 1 & 0 & 0 \\
$(5,7,5)$ & 8 & 0 & 0 & 8 & 8 & 8 & 8 & 8 \\
$(5,5,7)$ & 0 & 0 & 0 & 2 & 0 & 1 & 1 & 0 \\
$(7,7,7)$ & 8 & 0 & 12 & 4 & 3 & 3 & 3 & 1 \\
$(10,5,5)$ & 10 & 0 & 2 & 3 & 2 & 1 & 1 & 0 \\
$(5,10,5)$ & 4 & 0 & 0 & 5 & 4 & 4 & 4 & 3 \\
$(5,5,10)$ & 1 & 0 & 5 & 6 & 1 & 2 & 2 & 1 \\
$(10,7,7)$ & 15 & 0 & 15 & 11 & 11 & 4 & 4 & 0 \\
$(7,10,7)$ & 13 & 0 & 15 & 8 & 5 & 5 & 5 & 0 \\
$(7,7,10)$ & 14 & 0 & 15 & 5 & 2 & 4 & 3 & 0 \\
$(10,10,10)$ & 15 & 0 & 15 & 14 & 15 & 6 & 13 & 0 \\
$(15,10,10)$ & 15 & 0 & 15 & 15 & 15 & 0 & 15 & 0 \\
$(10,15,10)$ & 15 & 0 & 15 & 15 & 15 & 8 & 15 & 0 \\
$(10,10,15)$ & 15 & 0 & 15 & 15 & 15 & 4 & 15 & 0 \\
$(15,15,15)$ & 15 & 4 & 15 & 15 & 15 & 12 & 15 & 0 \\
\hline Total & 160 & 5 & 141 & 143 & 123 & 77 & 118 & 24 \\
\hline
\end{tabular}


de médio porte.

A Tabela 13 apresenta a média dos tempos computacionais dos procedimentos da literatura por classe. Apenas os tempos computacionais dos testes que obtiveram soluções factíveis são contabilizados. Enquanto isso, a Tabela 14 eem umera os problemas-teste não resolvidos de cada procedimento da literatura por classe de problema-teste. A última coluna da Tabela 14, a coluna Inf, indica os problemas-teste comprovadamente infactíveis enquanto a última linha da Tabela 14 apresenta a soma total de todas os problemasteste não resolvidos, por procedimento. A infactibilidade de alguns problemas-teste foi constatada por meio do software IBM ILOG CPLEX em um tempo menor que 7200 segundos.

A abordagem exata para problemas de pequeno porte é eficiente, dado o tempo computacional gasto (em média, tempo máximo de 17,07 segundos), todavia, para problemas de grande porte, não consegue em geral encontrar soluções factíveis no tempo máximo permitido. Para muitas classes de médio porte o procedimento de resolução exata tem média de tempo computacional gasto maior do que o tempo limite de 7200 segundos, o que implica que as soluções dessas classes não são comprovadamente ótimas. Com relação aos limitantes, nota-se a grande vantagem do limitante $L 1$, que salvo a classe $(15,15,15)$, precisa de menos de 10 minutos em média para encontrar um limitante.

As abordagens heurísticas da literatura possuem, de maneira geral, tempos computacionais melhores que o procedimento de resolução exata, com destaque para a heurística RH3 principalmente para os problemas-teste de grande porte, quando as outras heurísticas não são tão eficientes. A heurística $R H 3$ deixou de determinar soluções factíveis em 77 dos 300 problemas-teste, sendo que 24 são notadamente infactíveis, enquanto outras heurísticas resolveram um menor número de problemas-teste. Nas classes de pequeno e médio porte a eficiência das heurísticas com relação ao número de soluções factíveis é próxima, exceto para $R H 1$. Os resultados implicam que ao se fixar as decisões de dimensionamento de lotes de uma iteração para as seguintes pode não ser vantajoso, já que RH2 obtêm mais soluções factíveis que RH1.

Por último, pode-se inferir dos dados apresentados na Tabela 14 que a maneira como os problemas-teste foram gerados implica em problemas-teste mais restritos quando a combinação $(N, M, T)$ apresenta $M>N$ e $M>T$. O fato do conjunto de problemas-teste apresentar exemplares infactíveis (consequência das capacidades das máquinas utilizadas) implica que foram testados de problemas-teste mais restritos até aqueles cujas capacidades são mais "folgadas". Vale ressaltar que para problemas-teste cujo fator de capacidade é 
de $80 \%$ da capacidade mínima para o pior caso, todos os problemas-teste são factíveis.

Para uma comparação dos resultados computacionais dos procedimentos da literatura, utilizou-se os gráficos de perfil de desempenho descritos no trabalho de Dolan e Moré (2002) e também no Apêndice B. A Figura 17 apresenta o perfil de desempenho dos procedimentos: $M I P, R H 1, R H 2, R H 3$ e $R F$. Os procedimentos que obtiveram as melhores razões de desempenho um maior número de vezes têm como melhor procedimento a própria resolução exata do problema $(M I P)$, seguida dos procedimentos $R H 2$ e $R H 3$. O procedimento que mais encontrou soluções factíveis (ao final do perfil de desempenho) foi notadamente o procedimento RH3, que tem uma eficiência maior para obter soluções para classes de problemas-teste de grande porte. Vale ressaltar que o procedimento de resolução exata do problema resolve menos da metade dos problemas-teste, enquanto RH3 resolve cerca de $75 \%$ dos problemas-teste. Se analisada a inclinação das curvas de desempenho de cada procedimento, pode-se inferir que o procedimento $R H 2$ tem razões de desempenho mais próximas da melhor razão, dada a inclinação de sua curva, para um valor de $\tau$ reduzido, diferentemente da curva de desempenho dos procedimentos $R H 1$, $R H 3$ е $R F$.

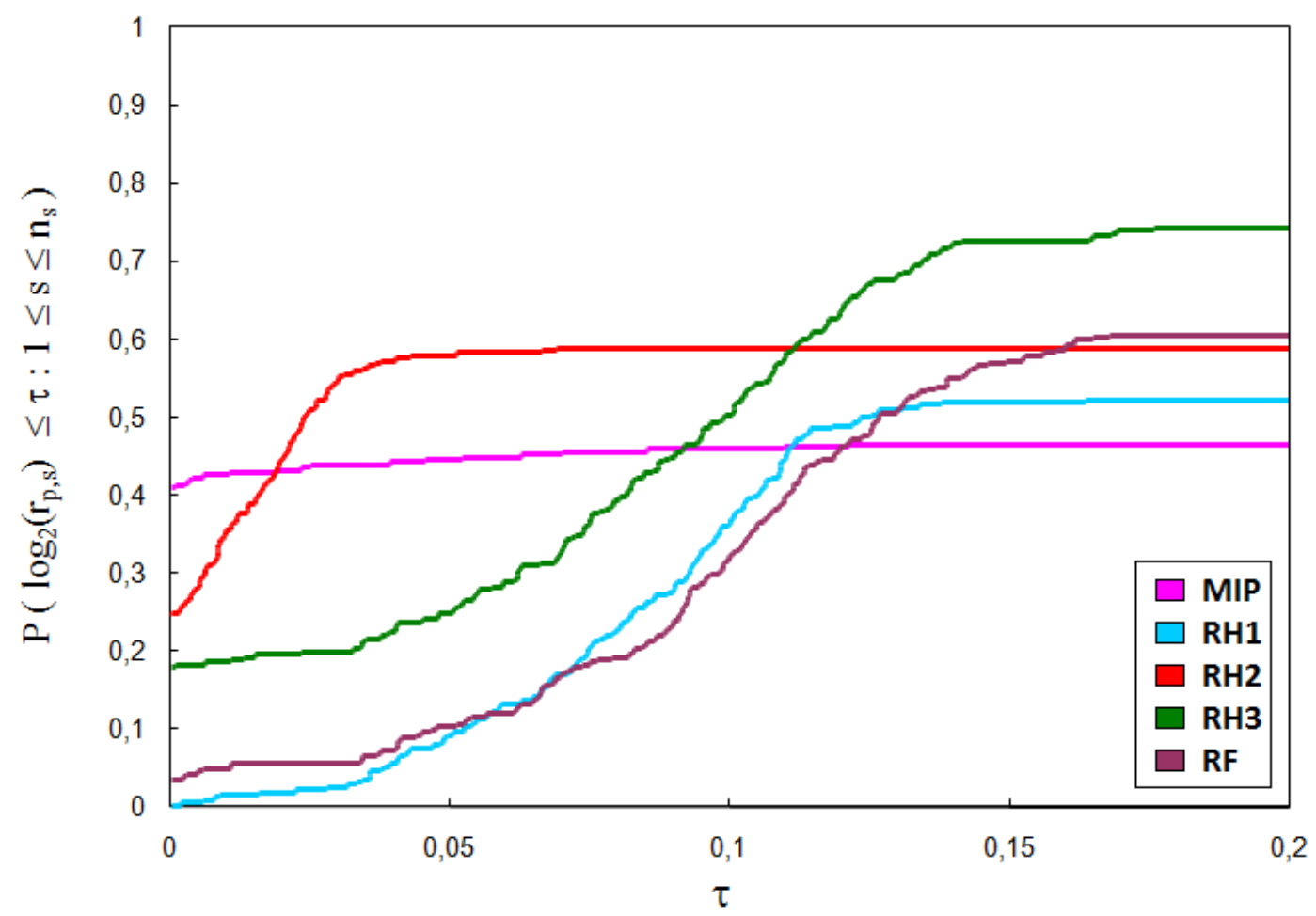

Figura 17: Perfil de Desempenho dos procedimentos avaliados. 


\subsubsection{Metaheurísticas Propostas}

Na Seção 5 dois tipos de metaheurísticas são propostos, uma das metaheurísticas se utiliza apenas de procedimentos heurísticos enquanto a outra é híbrida e possui procedimentos heurísticos e exatos ( $A T$ e $A T H$ respectivamente). Diversos agentes são apresentados, com ênfase em agentes construtores e de melhoria. Os agentes construtores são dados pelos agentes: $A g C 1, A g C 2, H A g C 1$ e $H A g C 2$. Vale ressaltar que os dois últimos agentes construtores se utilizam de procedimentos exatos. Os agentes de melhoria são divididos de acordo com o foco do agente de melhoria. Agentes de melhoria que tomam decisões relacionadas à sequência de produção são dados por: $A g S w, A g I n, A g B I$ e $A g C O$. Outra divisão de agentes de melhoria tem foco nas decisões de dimensionamento de lotes e são representados pelos agentes: $A g A P, A g E P, A g A E P, A g E P 1, A g A E P 1, A g E P \mathscr{2}$ e AgAEP2.

Pela diversidade dos agentes, várias configurações foram utilizadas visando obter uma metaheurística mais robusta. As configurações testadas são apresentadas na Tabela 15. Seis metaheurísticas do tipo $A T$ e duas do tipo $A T H$ são testadas. A diferença entre as heurísticas do tipo $A T$ reside na configuração dos agentes de melhoria com foco no problema de dimensionamento de lotes. Por exemplo a metaheurística AT6 utiliza todos os agentes de melhoria apresentados, enquanto a metaheurística AT3 apenas dos agentes $A g E P$ e $A g A E P$. A diferença entre as metaheurísticas ATH1 e ATH2 está na capacidade das memórias compartilhadas. Por utilizar procedimentos exatos, as metaheurísticas híbridas tendem a consumir mais tempo computacional. Por isso, a metaheurística ATH2 utilizou vinte soluções na memória compartilhada enquanto ATH1 utilizou apenas dez. A metaheurística $A T 4$ utiliza os mesmos agentes de AT3, porém, os procedimentos $A g E P$ e $A g A E P$ têm um pequena modificação que possibilita o término da atuação do agente mais cedo.

Perfis de desempenho também foram utilizados para comparar as metaheurísticas propostas entre si. O primeiro perfil de desempenho, ilustrado na Figura 18, apresenta uma comparação entre as metaheurísticas propostas AT1, AT2, AT3, AT4, AT5 e AT6. O perfil de desempenho da Figura 18 demonstra que o procedimento AT3 domina os outros procedimentos, tanto por ser o procedimento que mais vezes obteve melhor desempenho frente aos outros, como é aquele que encontra mais soluções factíveis dentre todos os procedimentos. Dessa forma, a metaheurística AT3 é a melhor configuração de $A T$, para os problemas-teste apresentados. Com relação à factibilidade das soluções finais, a metaheurística AT3 encontrou em $78 \%$ dos problemas-teste uma solução factível. 
Tabela 15: Configuração geral das principais metaheurísticas.

\begin{tabular}{|c|cccccccc|}
\hline Agentes & $A T 1$ & $A T 2$ & $A T 3$ & $A T 4$ & $A T 5$ & $A T 6$ & $A T H 1$ & $A T H 2$ \\
\hline$A g C 1$ & $\mathrm{x}$ & $\mathrm{x}$ & $\mathrm{x}$ & $\mathrm{x}$ & $\mathrm{x}$ & $\mathrm{x}$ & & \\
$A g C 2$ & $\mathrm{x}$ & $\mathrm{x}$ & $\mathrm{x}$ & $\mathrm{x}$ & $\mathrm{x}$ & $\mathrm{x}$ & & \\
$H A g C 1$ & & & & & & & $\mathrm{x}$ & $\mathrm{x}$ \\
$H A g C 2$ & & & & & & & $\mathrm{x}$ & $\mathrm{x}$ \\
\hline$A g D$ & $\mathrm{x}$ & $\mathrm{x}$ & $\mathrm{x}$ & $\mathrm{x}$ & $\mathrm{x}$ & $\mathrm{x}$ & $\mathrm{x}$ & $\mathrm{x}$ \\
\hline$A g S w$ & $\mathrm{x}$ & $\mathrm{x}$ & $\mathrm{x}$ & $\mathrm{x}$ & $\mathrm{x}$ & $\mathrm{x}$ & $\mathrm{x}$ & $\mathrm{x}$ \\
$A g I n$ & $\mathrm{x}$ & $\mathrm{x}$ & $\mathrm{x}$ & $\mathrm{x}$ & $\mathrm{x}$ & $\mathrm{x}$ & $\mathrm{x}$ & $\mathrm{x}$ \\
$A g B I$ & $\mathrm{x}$ & $\mathrm{x}$ & $\mathrm{x}$ & $\mathrm{x}$ & $\mathrm{x}$ & $\mathrm{x}$ & $\mathrm{x}$ & $\mathrm{x}$ \\
$A g C O$ & $\mathrm{x}$ & $\mathrm{x}$ & $\mathrm{x}$ & $\mathrm{x}$ & $\mathrm{x}$ & $\mathrm{x}$ & $\mathrm{x}$ & $\mathrm{x}$ \\
$A g A P$ & $\mathrm{x}$ & $\mathrm{x}$ & $\mathrm{x}$ & $\mathrm{x}$ & $\mathrm{x}$ & $\mathrm{x}$ & $\mathrm{x}$ & $\mathrm{x}$ \\
$A g E P$ & & & $\mathrm{x}$ & $\mathrm{x}$ & $\mathrm{x}$ & $\mathrm{x}$ & $\mathrm{x}$ & $\mathrm{x}$ \\
$A g A E P$ & & & $\mathrm{x}$ & $\mathrm{x}$ & $\mathrm{x}$ & $\mathrm{x}$ & $\mathrm{x}$ & $\mathrm{x}$ \\
$A g E P 1$ & & $\mathrm{x}$ & & & & $\mathrm{x}$ & & \\
$A g A E P 1$ & & $\mathrm{x}$ & & & & $\mathrm{x}$ & & \\
$A g E P 2$ & $\mathrm{x}$ & & & & $\mathrm{x}$ & $\mathrm{x}$ & & \\
$A g A E P 2$ & $\mathrm{x}$ & & & & $\mathrm{x}$ & $\mathrm{x}$ & & \\
\hline
\end{tabular}

O perfil de desempenho apresentado na Figura 19 relaciona as metaheurísticas híbridas propostas ATH1 e ATH2 com a metaheurística AT3. Comparando apenas as metaheurísticas Times Assíncronos híbridas, um domínio do procedimento ATH2 sobre ATH1 é apresentado. A única diferença entre esses procedimentos é o número de soluções presentes na memória compartilhada. Dessa forma, pode-se inferir que um número maior de soluções na memória compartilhada apresenta melhores soluções. Porém, há um gasto maior com o tempo de construção de todas as soluções iniciais. Se comparado os procedimentos ATH2 e AT3, ou as melhores metaheurísticas propostas, percebe-se que AT3 obteve, para um maior número de problemas-teste, mais vezes um melhor desempenho, entretanto a metaheurística ATH2 consegue encontrar uma solução factível para um número maior de problemas-teste. A metaheurística ATH2 obteve soluções factíveis em $82 \%$ dos problemas-teste. Dado que 24 são infactíveis, ou $8 \%$ dos 300 problemas-teste, $10 \%$ dos problemas-teste não se sabe se são factíveis ou não.

Como dito anteriormente, todos os testes computacionais de metaheurísticas foram simulados cinco vezes. O tempo computacional do procedimento é dado pela somatória dos tempos de cada simulação. A Tabela 16 apresenta a média dos tempos computacionais das metaheurísticas com relação às classes de problemas-teste. O tempo computacional gasto pela metaheurística ATH1 é menor que o tempo consumido por ATH2. Esse resultado era esperado pois o número de soluções geradas pelos agentes construtores em ATH1 é menor. As metaheurísticas não-híbridas consomem tempo geralmente menor que a 


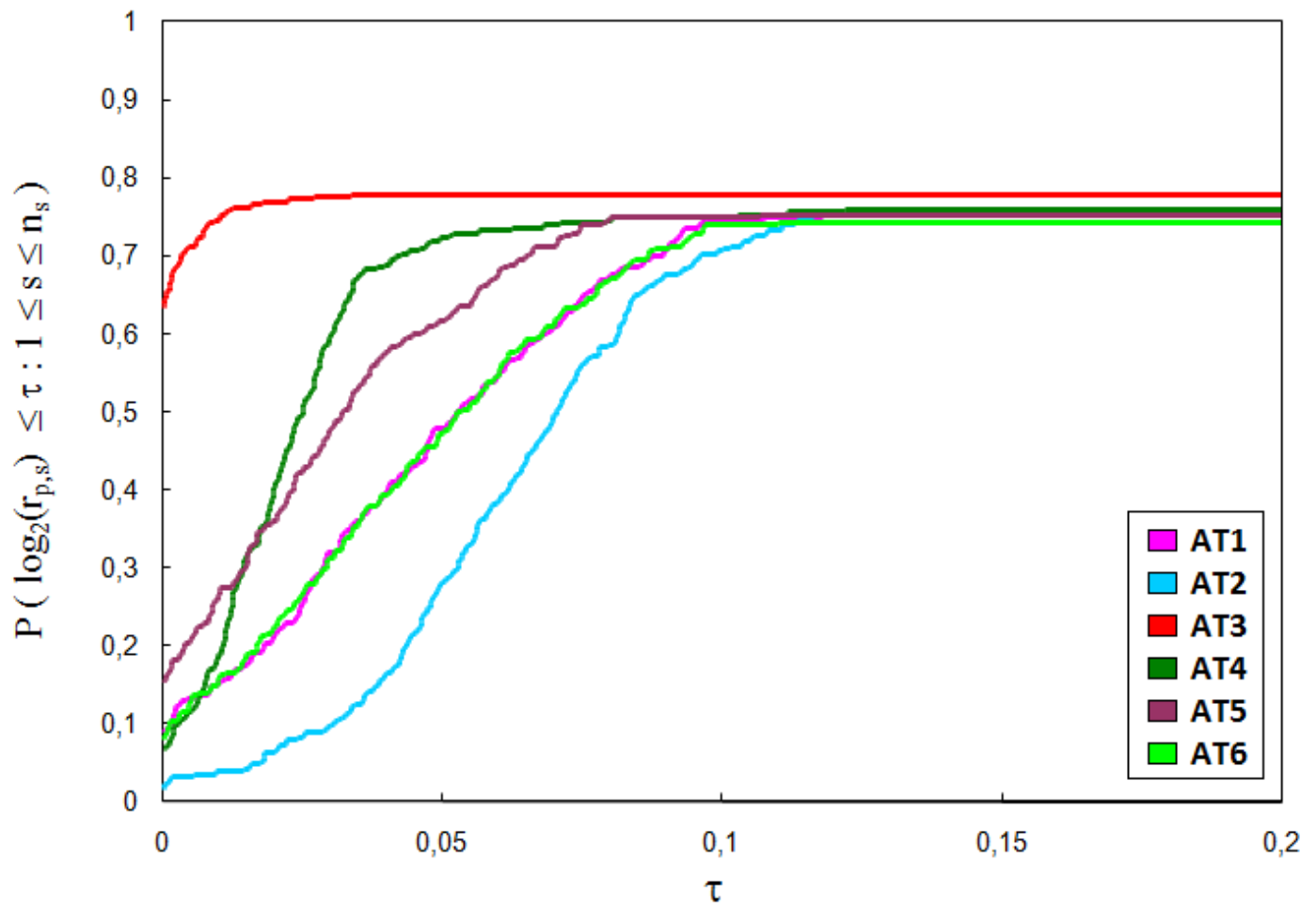

Figura 18: Perfil de Desempenho das metaheurísticas times assíncronos.

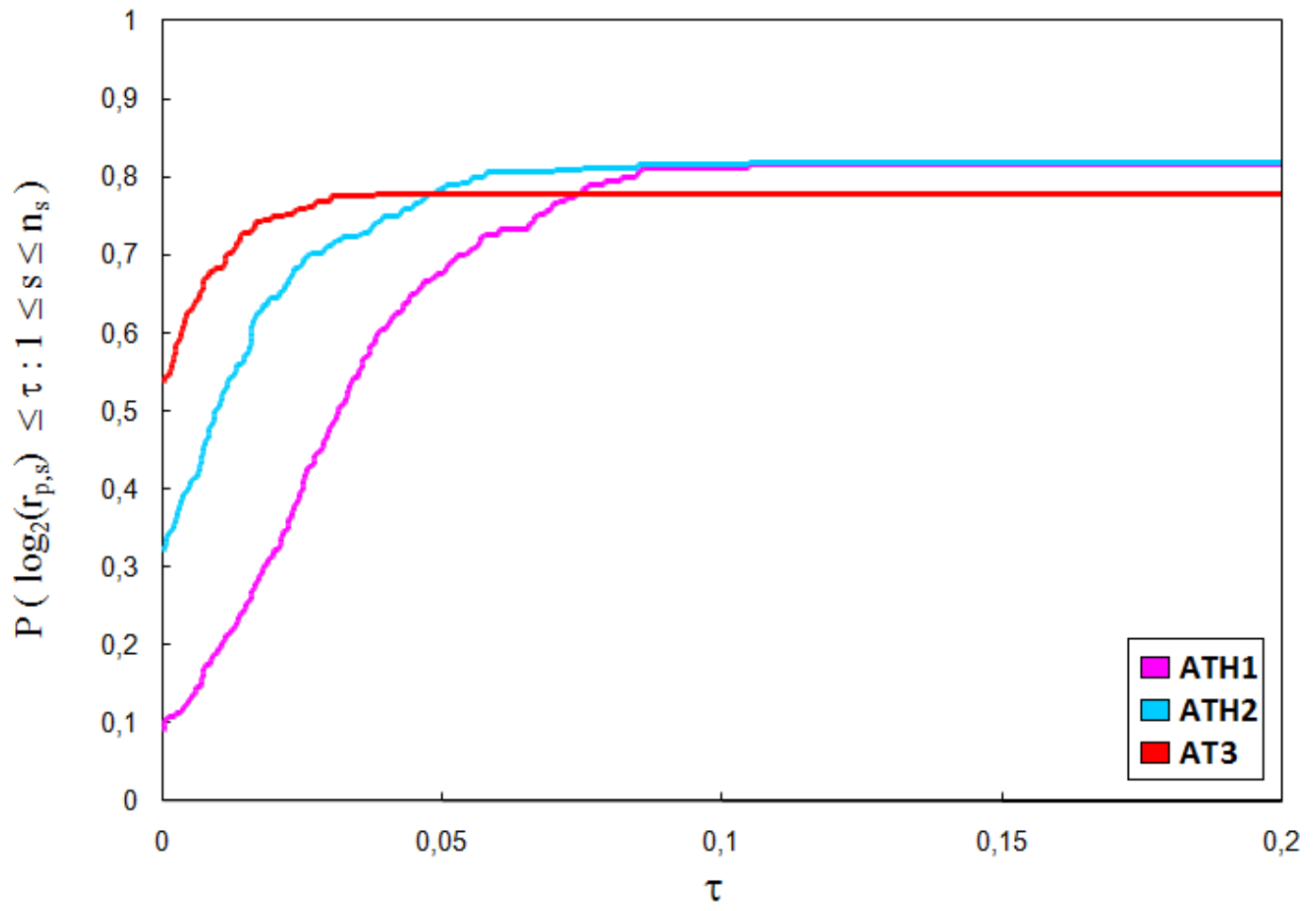

Figura 19: Perfil de Desempenho das metaheurísticas times assíncronos híbridas. 
metaheurística híbrida. De acordo com os resultados, os agentes de melhoria AgEP1 e AgAEP1 consomem muito tempo computacional, e as suas soluções não são tão boas. Os agentes que determinam o adiantamento da produção de produtos acabados (AgEP e $A g A E P)$ obtiveram melhores resultados que os agentes que atuam com a geração de soluções com estoques intermediários ( $A g E P 1, A g E P 2, A g A E P 1$ e $A g A E P 2)$.

Tabela 16: Média dos tempos computacionais por classe de problema-teste.

\begin{tabular}{|c|cccccccc|}
\hline & $A T 1$ & $A T 2$ & $A T 3$ & $A T 4$ & $A T 5$ & $A T 6$ & $A T H 1$ & $A T H 2$ \\
\hline$(3,3,3)$ & 7,78 & 8,33 & 18,41 & 13,09 & 6,94 & 7,93 & 2,98 & 17,36 \\
$(5,3,3)$ & 10,00 & 16,31 & 12,47 & 17,32 & 9,14 & 11,99 & 2,13 & 17,59 \\
$(3,5,3)$ & 8,84 & 13,27 & 9,71 & 13,16 & 8,49 & 11,26 & 4,28 & 20,30 \\
$(3,3,5)$ & 13,53 & 14,08 & 18,12 & 20,08 & 10,77 & 12,23 & 2,87 & 23,23 \\
$(5,5,5)$ & 23,44 & 45,59 & 25,62 & 31,05 & 21,51 & 35,13 & 3,81 & 54,62 \\
$(7,5,5)$ & 37,40 & 73,93 & 28,32 & 39,84 & 33,24 & 80,41 & 5,09 & 53,09 \\
$(5,7,5)$ & 37,11 & 76,75 & 28,12 & 32,70 & 32,10 & 57,35 & 4,26 & 35,67 \\
$(5,5,7)$ & 31,79 & 68,45 & 41,82 & 50,52 & 32,40 & 43,86 & 5,76 & 86,02 \\
$(7,7,7)$ & 86,86 & 192,29 & 47,58 & 69,79 & 59,49 & 142,87 & 8,75 & 74,23 \\
$(10,5,5)$ & 59,54 & 134,64 & 43,54 & 59,21 & 58,53 & 102,74 & 8,41 & 97,71 \\
$(5,10,5)$ & 44,23 & 190,65 & 24,32 & 34,11 & 33,03 & 155,86 & 6,35 & 55,87 \\
$(5,5,10)$ & 48,67 & 98,81 & 66,00 & 77,45 & 37,39 & 86,52 & 12,17 & 72,68 \\
$(10,7,7)$ & 111,75 & 312,48 & 67,47 & 90,41 & 93,77 & 216,51 & 13,93 & 102,60 \\
$(7,10,7)$ & 111,14 & 305,19 & 51,55 & 82,70 & 76,04 & 261,70 & 12,75 & 98,49 \\
$(7,7,10)$ & 103,29 & 211,18 & 66,81 & 99,78 & 74,93 & 173,52 & 15,50 & 127,90 \\
$(10,10,10)$ & 217,78 & 790,29 & 98,71 & 168,78 & 195,08 & 619,88 & 33,50 & 195,83 \\
$(15,10,10)$ & 414,44 & 1419,17 & 147,80 & 232,60 & 342,29 & 1217,40 & 68,54 & 329,42 \\
$(10,15,10)$ & 291,35 & 1279,23 & 116,91 & 193,69 & 289,43 & 1669,11 & 69,02 & 329,39 \\
$(10,10,15)$ & 347,27 & 1403,18 & 159,19 & 256,11 & 295,49 & 1117,90 & 73,10 & 372,53 \\
$(15,15,15)$ & 738,45 & 3919,19 & 323,44 & 499,10 & 707,08 & 4419,41 & 457,95 & 1494,76 \\
\hline
\end{tabular}

\subsubsection{Resultados Finais}

Nesta seção os procedimentos principais da literatura são confrontados com as metaheurísticas propostas. O perfil de desempenho final, ilustrado na Figura 20, compara os procedimentos MIP, RH2, RH3 e os melhores procedimentos propostos (metaheurísticas AT3 e ATH2). Dentre os procedimentos que obtiveram as melhores razões de desempenho um maior número de vezes, se destaca a própria resolução exata do problema $M I P$, seguida dos procedimentos RH2, AT3 e ATH2. Se observado os procedimentos que mais encontraram soluções factíveis, as metaheurísticas propostas são mais robustas, seguidas do procedimento RH3. Se analisada a inclinação das curvas de desempenho de cada procedimento, pode-se inferir que os procedimentos RH2, AT3 e ATH2 têm razões de desempenho mais próximas da melhor razão, dada a inclinação de suas curvas, para um valor de $\tau$ reduzido, diferentemente da curva de desempenho do procedimento $R H 3$.

Com relação ao tempo computacional de todos os procedimentos vistos nas Tabelas 13 e 16, a heurística $R H 3$ possui um bom tempo computacional para problemas-teste de pequeno e médio porte, comparado aos outros procedimentos, porém, para problemas de grande porte os tempos computacionais das metaheurísticas, principalmente o procedimento AT3, são mais competitivos. Em geral, os tempos dos procedimentos propostos AT3 e ATH2 não são bons para problemas-teste de pequeno porte. 


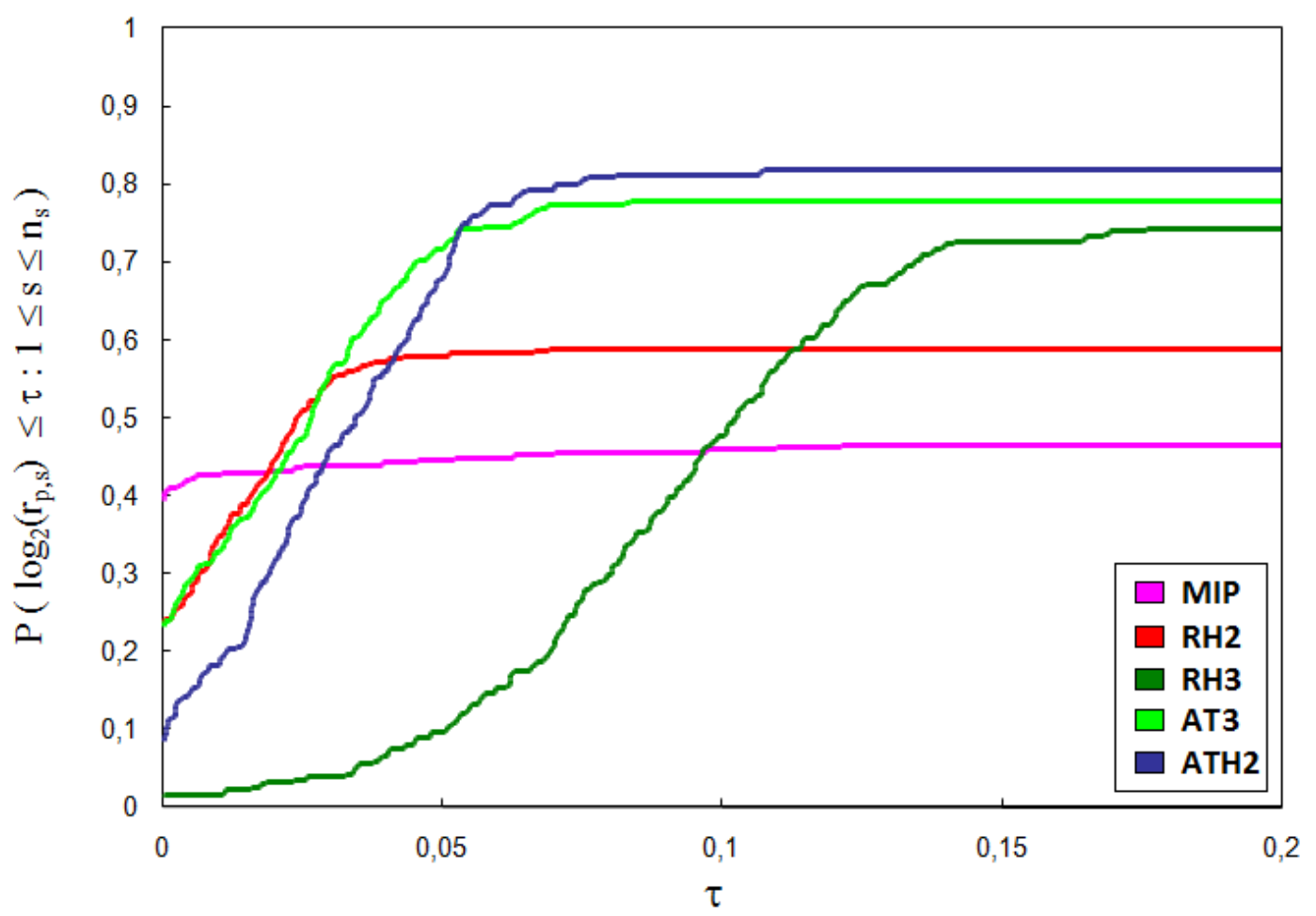

Figura 20: Perfil de Desempenho dos procedimentos avaliados. 


\section{Conclusões e Perspectivas}

Neste trabalho o problema integrado de programação da produção e dimensionamento de lotes em um ambiente de produção flowshop é tratado. O problema considera que a demanda deve ser atendida sem atrasos, que as capacidades das máquinas devem ser respeitadas, que as preparações de máquinas são dependentes da sequência e são também preservadas entre períodos do horizonte de planejamento. O objetivo é a minimização do custo de produção, preparação de máquinas e estoque. Modelos matemáticos propostos por Mohammadi et al. (2010a) e Mohammadi et al. (2010b) abordam esse problema. Estas formulações foram analisadas e foi escolhido o modelo matemático cujas soluções abordam apenas soluções permutacionais, já que são prática na indústria e na literatura.

Limitantes e heurísticas presentes na literatura foram revistos, avaliados, adaptados e comparados. As heurísticas abordadas utilizam procedimentos distintos, como a estratégia relax-and-fix e a estratégia do horizonte rolante. Todas heurísticas da literatura possuem procedimentos exatos inclusos, o que faz com que algumas delas não sejam tão eficientes para problemas de grande porte, no sentido de que mesmo soluções factíveis não são encontradas em tempo hábil.

Procedimentos de solução metaheurísticos, baseados na metaheurística Times Assíncronos, foram propostos e, juntamente com procedimentos da literatura, avaliados com um conjunto de problemas-teste gerado. Primeiramente, uma metaheurística baseada em Times Assíncronos foi proposta. Diversos agentes heurísticos foram implementados e diversas configurações testadas. As principais configurações foram apresentadas nesta dissertação e obtiveram bons resultados. Feito isto, foi proposta a hibridização desta metaheurística a partir da utilização de agentes com uma estratégia que envolve algum tipo de procedimento exato. Isso tornou a metaheurística proposta mais robusta, conseguindo obter mais soluções factíveis em tempo computacional um pouco pior que a metaheurística não-híbrida.

Ao se comparar as metaheurísticas propostas entre si, duas se destacaram, as meta- 
heurísticas AT3 e ATH2. Ao contrário do que se esperava, agentes que buscavam soluções com estoques intermediários não se destacaram. Comparando as metaheurísticas propostas com os procedimentos da literatura, concluiu-se que as metaheurísticas AT3 e ATH2, principalmente para problemas-teste de médio e grande porte, obtiveram bons resultados, dado que ambas resolveram muitos problemas não resolvidos pelos procedimentos da literatura, além de obter soluções com qualidades equivalentes à qualidade das soluções obtidas pelos métodos da literatura. Assim, a utilização das metaheurísticas deve ser encorajada, o que atesta uma das perspectivas futuras dos trabalhos relacionados Mohammadi et al. (2010a, 2010b).

Como perspectivas futuras, novas características podem ser agregadas ao modelo matemático original de Mohammadi et al. (2010a), produzindo formulações matemáticas derivadas e estendendo o modelo matemático para se tornar mais fiel à realidade. Por exemplo, linhas de produção que consideram o bloqueio de máquinas (blocking) por falta de espaço para estoques intermediários, ambientes produtivos que permitem a divisão de um lote de produtos (lot splitting) ou mesmo considerar um problema de flowshop flexível, em que cada estágio de produção pode ter mais do que somente uma máquina.

Além disso, métodos de solução heurísticos sempre podem ser alterados e melhorados. Outras estratégias podem ser avaliadas, como por exemplo a utilização de hiperheurísticas, outros procedimentos exatos, dentre outros. Uma maneira de tornar a metaheurística mais sofisticada, por exemplo, é utilizar todos os recursos disponíveis de processamento distribuído, como processadores de vários núcleos, ou mesmo a utilização de unidades de processamento gráfico, as GPUs. 


\section{Referências Bibliográficas}

ALLAHVERDI, A.; GUPTA, J.; ALDOWAISAN, T. A survey of scheduling research involving setup considerations. OMEGA, International Journal of Management Science, v. 27, p. 219-239, 1999.

ASHOUR, S. Sequencing Theory. [S.l.]: Springer Verlag, Berlin, 1972.

BAHL, H. C.; RITZMAN, L. P.; GUPTA, J. N. Determining lot sizes and resource requirements: A review. Oper. Res., INFORMS, Institute for Operations Research and the Management Sciences (INFORMS), Linthicum, Maryland, USA, v. 35, n. 3, p. 329-345, 1987.

BAKER, K. R. Introduction to Sequencing and Scheduling. [S.l.]: John Wiley, 1974.

BAKER, K. R.; TRIETSCH, D. Principles of Sequencing and Scheduling. [S.l.]: John Wiley and Sons, 2009.

BELO FILHO, M.; SANTOS, M.; MENESES, C. Times assíncronos aplicados ao problema de dimensionamento e sequenciamento de lotes de produtos em um ambiente do tipo flow shop com estoques intermediários e restrições de capacidade. Revista de Iniciação Científica do Centro de Tecnologia Educacional para Engenharia da Escola de Engenharia de São Carlos - USP, v. 8, p. 75-82, 2009.

BITRAN, G.; YANASSE, H. Computational complexity of the capacitated lot size problem. Management Science, v. 28, n. 10, p. 1174-1186, 1982.

BODT, M. A. D.; GELDERS, L. F.; WASSENHOVE, L. N. V. Lot sizing under dynamic demand conditions: A review. Engineering Costs and Production Economics, v. 8, p. $165-187,1984$.

BRAHIMI, N.; DAUZERE-PERES, S.; NAJID, N. M.; NORDLI, A. Single item lot sizing problems. European Journal of Operational Research, v. 168, n. 1, p. 1-16, 2006.

BRISKORN, D. A note on capacitated lot sizing with setup carry over. IIE Transactions, v. 38, n. 11, p. 1045-1047, 2006.

CHENG, T. C. E.; GUPTA, J. N. D.; WANG, G. A review of flowshop scheduling research with setup times. Production and Operations Management, v. 9, n. 3, p. 262-282, 2000.

CONWAY, R. L.; MAXWELL, W. L.; MILLER, L. W. Theory of Scheduling. Reading, MA: Addison Wesley, 1967.

DAUZèRE-PéRES, S.; LASSERRE, J. Integration of lotsizing and scheduling decisions in a job-shop. European Journal of Operational Research, v. 75, p. 413-426, 1994. 
DE SOUZA, P.; TALUKDAR, S. Asynchronous organizations for multialgorithm problems. ACM Symposium on Applied Computing, Indianapolis, 1993.

DE SOUZA, P. S. Asynchronous organizations for multi-algorithm problems. Tese (Doutorado), Pittsburgh, PA, USA, 1993.

DOLAN, E. D.; MORé, J. J. Benchmarking optimization software with performance profiles. Mathematical Programming, v. 91, n. 2, p. 201-213, 2002.

DREXL, A.; KIMMS, A. Lot sizing and scheduling: Survey and extensions. European Journal of Operational Research, v. 99, n. 2, p. 221-235, 1997.

FANDEL, G.; STAMMEN-HEGENE, C. Simultaneous lot sizing and scheduling for multi-product multi-level production. International Journal of Production Economics, v. 104, n. 2, p. 308-316, 2006.

GAREY, M. R.; JOHNSON, D. S.; SETHI, R. The complexity of flowshop and jobshop scheduling. Mathematics of Operations Research, v. 1, n. 2, p. 117-129, 1976.

GOPALAKRISHNAN, M.; DING, K.; BOURJOLLY, J.-M.; MOHAN, S. A tabu-search heuristic for the capacitated lot-sizing problem with set-up carryover. Management Science, v. 47, p. 851-863, 2001.

GOPALAKRISHNAN, M.; MILLER, D.; SCHMIDT, C. P. A framework for modelling setup carryover in the capacitated lot sizing problem. International Journal of Production Research, v. 38, p. 3421-3424, 2000.

GOPALAKRISHNAN, M.; MILLER, D. M.; SCHMIDT, C. P. A framework for modelling setup carryover in the capacitated lot sizing problem. International Journal of Production Research, v. 33, p. 1973-1988, 1995.

GRAHAM, R. L.; LAWLER, E. L.; LENSTRA, J. K.; KAN, A. H. G. R. Optimization and approximation in deterministic sequencing and scheduling: a survey. Annals of discrete Mathematics, v. 5, p. 287-326, 1979.

GUPTA, J.; STAFFORD JR., E. Flowshop scheduling research after five decades. European Journal of Operational Research, v. 169, p. 699-711, 2006.

HARRIS, F. How many parts to make at once. The Magazine of Management, v. 10, p. 135-136, 1913.

JANS, R.; DEGRAEVE, Z. Meta-heuristics for dynamic lot sizing: A review and comparison of solution approaches. European Journal of Operational Research, v. 177, p. 1855-1875, 2007.

JANS, R.; DEGRAEVE, Z. Modeling industrial lot sizing problems: a review. International Journal of Production Research, v. 46, p. 1619-1643, 2008.

JOHNSON, S. M. Optimal two- and three-stage production schedules with setup times included. Naval Research Logistics Quarterly, v. 1, p. 61-68, 1954.

KARIMI, B.; FATEMI GHOMI, S. M. T.; WILSON, J. M. The capacitated lot sizing problem: a review of models and algorithms. Omega, v. 31, p. 365-378, 2003. 
KARIMI, B.; FATEMI GHOMI, S. M. T.; WILSON, J. M. A tabu search heuristic for solving the clsp with backlogging and set-up carry-over. Journal of the Operational Research Society, v. 57, p. 140-147, 2006.

KUIK, R.; SALOMON, M.; WASSENHOVE, L. N. V. Batching decisions: structure and models. European Journal of Operational Research, v. 75, p. 243-263, 1994.

LAWLER, E. L.; LENSTRA, J. K.; KAN, A. H. G. R.; SHMOYS, D. B. Handbooks in operations research and management science. In: [S.l.]: Elsevier Science Publishers, Amsterdam, 1993. v. 4, cap. Sequencing and scheduling: Algorithms and complexity, p. $445-552$.

LEE, I.; SIKORA, R.; SHAW, M. J. A genetic algorithm-based approach to flexible flow-line scheduling with variable lot sizes. IEEE Transactions on Systems, Man, and Cybernetics, Part B, v. 27, n. 1, p. 36-54, 1997.

MANNE, A. Programming of economic lot sizes. Management Science, v. 4, p. 115-135, 1958.

MENESES, C. N.; PARDALOS, P. M.; RAGLE, M. Asynchronous teams for probe selection problems. Discrete Optimization, v. 5, n. 1, p. $74-87,2008$.

MERCé, C.; FONTAN, G. Mip-based heuristics for capacitated lotsizing problems. International Journal of Production Economics, v. 85, n. 1, p. 97-111, 2003.

Disponível em: <http://www.sciencedirect.com/science/article/B6VF8-48VW9PS4/2/7390d27d3d3cd6cced305b663393983b>.

MOHAMMADI, M.; FATEMI GHOMI, S.; KARIMI, B.; TORABI, S. Rolling-horizon and fix-and-relax heuristics for the multi-product multi-level capacitated lotsizing problem with sequence-dependent setups. Journal of Intelligent Manufacturing, 2008. Disponível em: <http://www.springerlink.com/content/025pv712736nv585>.

MOHAMMADI, M.; FATEMI GHOMI, S.; KARIMI, B.; TORABI, S. Mip-based heuristics for lotsizing in capacitated pure flow shop with sequence-dependent setups. International Journal of Production Research, Taylor \& Francis, v. 48, n. 10, p. 2957-2973, 2010. Disponível em: <http://www.informaworld.com/10.1080/00207540902796016>.

MOHAMMADI, M.; FATEMI GHOMI, S.; KARIMI, B.; TORABI, S. A new algorithmic approach for capacitated lot-sizing problem in flow shops with sequence-dependent setups. The International Journal of Advanced Manufacturing Technology, v. 49, p. 201-211, 2010. Disponível em: <http://www.springerlink.com/content/nr7424nj74l61532>.

MORRIS, P. W. G. The management of projects. [S.l.]: Thomas Telford, 1997.

NAWAZ, M.; ENSCORE JR., E. E.; HAM, I. A heuristic algorithm for the m-machine, n-job flow-shop sequencing problem. Omega - The International Journal of Management Science, v. 1, p. 91-95, 1983.

PALMER, D. S. Sequencing jobs through a multistage process in the minimum total time: a quick method of obtaining a near optimum. Operations Research Quarterly, v. 16 , p. 101-107, 1965. 
PARK, S.; MILLER, K. Random number generators: Good ones are hard to find. Communications of the ACM, v. 31, p. 1192-1201, 1988.

PINEDO, M. Scheduling: Theory, Algorithms and Systems. 3rd edition. ed. [S.l.]: Springer, 2008.

POCHET, Y.; WOLSEY, L. Production Planning by Mixed Integer Programming. [S.1.]: Springer, 2006.

PONNAMBALAM, S. G.; REDDY, M. M. A ga-sa multiobjective hybrid search algorithm for integrating lot sizing and sequencing in flow-line scheduling. International Journal if Advanced Manufactoring Technology, v. 21, p. 126-137, 2003.

PORKKA, P.; VEPSäLäINEN, A. P. J.; KUULA, M. Multiperiod production planning carryign over set-up time. International Journal of Production Research, v. 41, p. 1133-1148, 2003.

POTTS, C.; WASSENHOVE, L. V. Integrating scheduling with batching and lot-sizing: a review of algorithms and complexity. Journal of Operational Research Society, v. 43, n. 5, p. 395-406, 1992.

POTTS, C. N.; SHMOYS, D. B.; WILLIAMSON, D. P. Permutation vs. non-permutation flow shop schedules. Operations Research Letters, v. 10, p. 281-284, 1991.

QUADT, D.; KUHN, H. A taxonomy of flexible flow line scheduling procedures. European Journal of Operational Research, v. 178, p. 686-698, 2007.

SIKORA, R. A genetic algorithm for integrating lot-sizing and sequencing in scheduling a capacitated flow line. Computers and Industrial Engineering, v. 30, n. 4, p. 969-981, 1996.

SIKORA, R.; CHHAJED, D.; SHAW, M. J. Integrating the lot-sizing and sequencing decisions for scheduling a capacitated flow line. Computers and Industrial Engineering, v. 30, n. 4, p. 659-679, 1996.

SILVER, E. A.; MEAL, H. C. A heuristic selecting lot size requirement for the case of a deterministic time varying demand rate and discrete opportunities for replenishment. Production and Inventory Management, v. 14, p. 64-74, 1973.

SMITH-DANIELS, V.; RITZMAN, L. A model for lot sizing and sequencing in process industries. International Journal of Production Research, v. 26, p. 647-674, 1988.

SOX, C. R.; GAO, Y. The capacitated lot sizing problem with setup carry-over. IIE Transactions, v. 31, p. 173-181, 1999.

SUERIE, C.; STADLER, H. The capacitated lot-sizing problem with linked lot sizes. Management Science, v. 49, p. 1039-1054, 2003.

SUNG, C.; MARAVELIAS, C. T. A mixed-integer programming formulation for the general capacitated lot-sizing problem. Computers \&f Chemical Engineering, v. 32, p. 244-259, 2008. 
TALUKDAR, S.; BAERENTZEN, L.; A., G.; SOUZA, P. de. Asynchronous teams: Cooperation schemes for autonomous agents. Journal of Heuristics, v. 4, p. 295-321, 1998.

TALUKDAR, S.; MURTHY, S.; AKKIRAJU, R. Handbook of metaheuristics. In: . [S.1.]: Springer, 2003. cap. Asynchronous Teams, p. 537-556.

TALUKDAR, S.; PYO, S.; GIRAS, T. Asynchronous procedures for parallel processing. Power Apparatus and Systems, IEEE Transactions on, PAS-102, n. 11, p. 3652-3659, Nov. 1983.

TANAEV, V. S.; SOTSKOV, Y.; STRUSEVICH, V. Scheduling Theory: Multi-stage Systems. Dordrecht: Kluwer Academic Publishers, 1994.

WAGNER, H. M.; WHITIN, T. Dynamic version of the economic lot size model. Management Science, v. 5, p. 89-96, 1958.

WOLSEY, L. Integer Programming. [S.l.]: New York: Wiley, 1998.

ZHU, X.; WILHELM, W. E. Scheduling and lot sizing with sequence-dependent setup: A literature review. IIE Transactions, v. 38, p. 987-1007, 2006. 


\section{APÊENICE A - Gráficos de Gantt}

O gráfico de Gantt (Gantt Chart), desenvolvido por Henry Laurence Gantt (Figura 21) nos Estados Unidos para a programação da produção no Frankford Arsenal em 1917, é comumente conhecido e utilizado atualmente de maneira essencialmente inalterada (MORRIS, 1997).

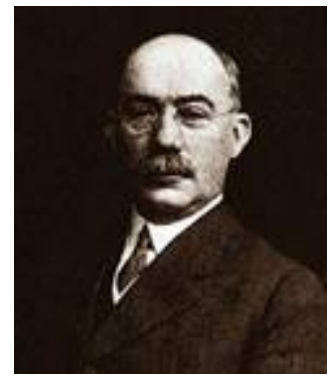

Figura 21: Rosto de Henry Gantt.

O gráfico de Gantt é uma representação análoga de uma programação. Na sua forma básica, o gráfico de Gantt exibe a alocação de recursos no tempo, com recursos específicos distribuídos no eixo vertical e uma escala de tempo no eixo horizontal. O gráfico de Gantt serve tanto como um auxílio para comparações entre programações como para visualização de detalhes do problema por meio da análise das relações geométricas presentes no gráfico (BAKER; TRIETSCH, 2009). Esse tipo de gráfico ilustra os instantes de ínicio e término de elementos da programação. O gráfico de Gantt é muito utilizado em gerenciamento de projetos e no planejamento de produção.

Exemplos de gráficos de Gantt estão ilustrados na figuras 22 e 23, que mostram a produção de três produtos em duas linhas de produção flowshop distintas. Cada estágio de produção (máquina) realiza uma tarefa representada por uma barra horizontal finita. Um produto é representado por uma única cor em todas as tarefas nas máquinas. Na primeira linha de produção (Figura 22) há um espaço intermediário entre as máquinas na qual produtos inacabados provenientes de tarefas completas podem esperar para serem produzidas na máquina seguinte. A segunda linha de produção (Figura 23) não possui 
tal espaço, determinando que a máquina cuja tarefa está completa seja bloqueada até que a máquina seguinte esteja disponível para realizar outra tarefa. O bloqueio de máquina é conhecida na literatura como blocking. Nesse exemplo, a terceira tarefa da segunda máquina da primeira linha de produção tem início no instante 60 e término no instante 90 e possui um tempo de processamento de 30 unidades de tempo. Na segunda programação, a mesma tarefa é atrasada pelo bloqueio da máquina $M 2$ (representada pela barra horizontal escura), assim, seu início é no instante 70. Uma visão global da programação de produção é facilmente visualizado. Nesse caso, podemos perceber que o tempo total de produção makespan é de 100 e 110 unidades de tempo, para as respectivas linhas de produção.

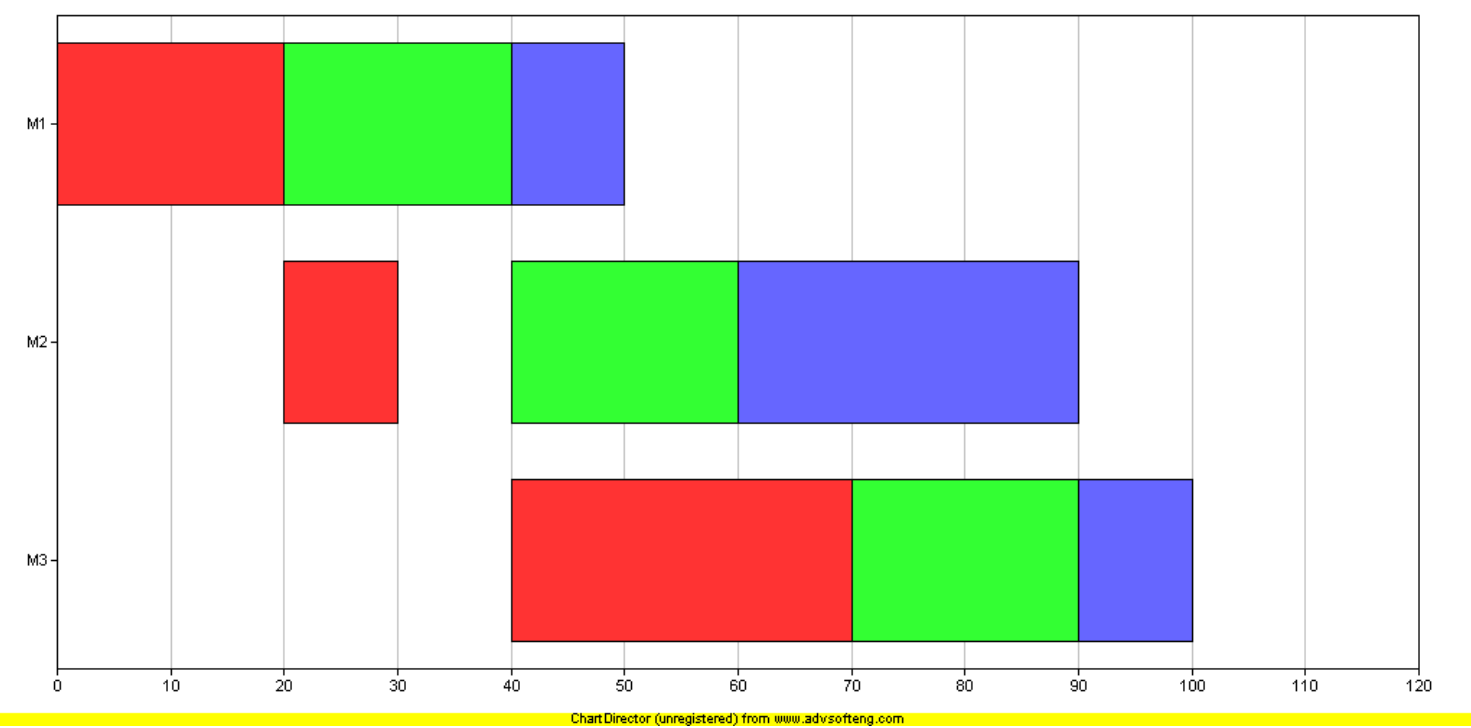

Figura 22: Gráfico de Gantt representando uma linha de produção sem blocking.

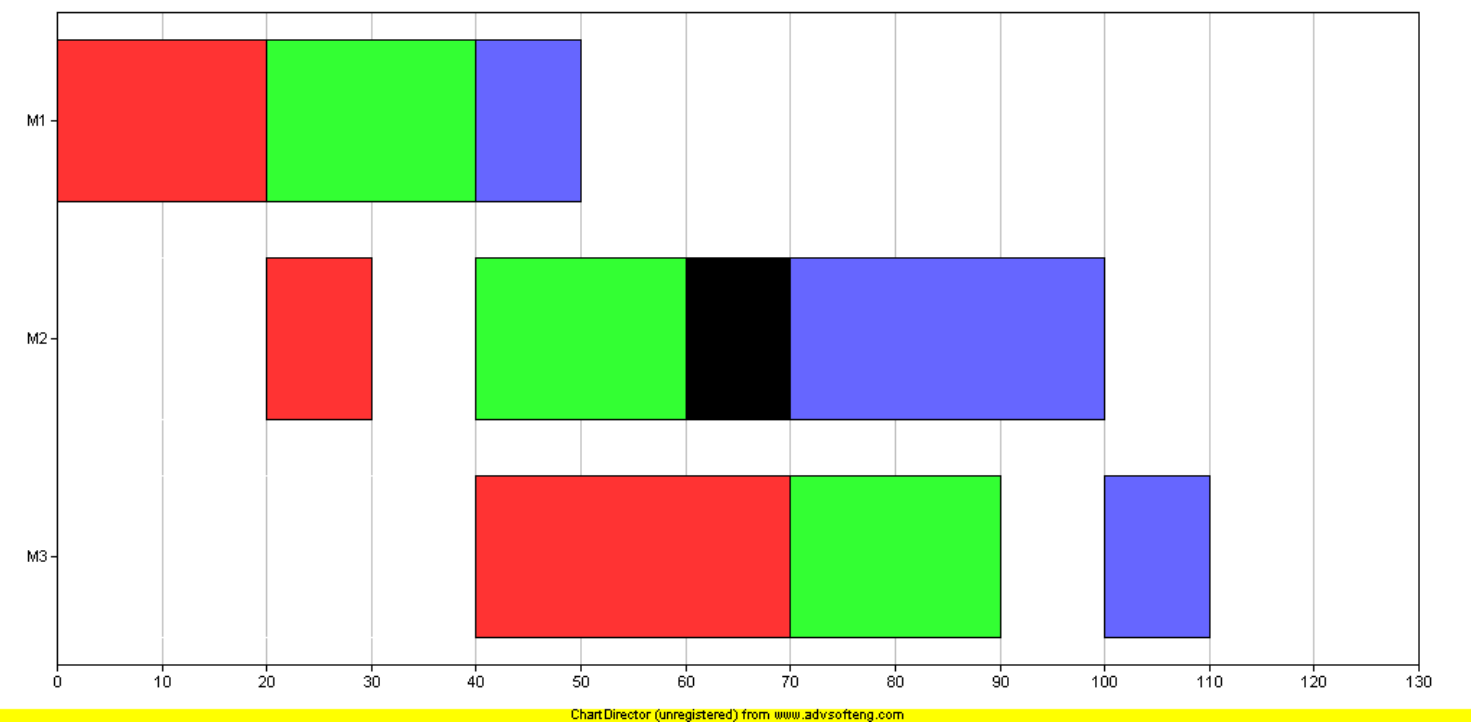

Figura 23: Gráfico de Gantt representando uma linha de produção com blocking. 


\section{APÊEDICE B - Perfil de Desempenho de Dolan e Moré (2002)}

O perfil de desempenho de Dolan e Moré é um gráfico onde métodos de solução podem ser comparados dado um conjunto de problemas-teste e uma medida de desempenho. Este tipo de gráfico está bem definido em Dolan e Moré (2002).

Seja $\mathcal{P}$ um conjunto de problemas-teste com $n_{p}$ elementos e $\mathcal{S}$ um conjunto de procedimentos de solução do conjunto $\mathcal{P}$ com $n_{s}$ procedimentos. Para se comparar esses procedimentos de solução utiliza-se uma medida de desempenho, por exemplo, tempo computacional gasto ou valor da solução encontrada. Definida a medida de desempenho, seja $t_{p, s}$ o resultado dessa medida no procedimento $s$ para o problema-teste $p$. A razão de desempenho pode ser obtida, para todo $p \in \mathcal{P}, s \in \mathcal{S}$ por meio da Equação B.1.

$$
r_{p, s}=\frac{t_{p, s}}{\min \left\{t_{p, s}: s \in \mathcal{S}\right\}}
$$

Assim, todos os procedimentos de solução são comparados para todos os problemasteste. Os problemas-teste nos quais algum procedimento não possui medida de desempenho tem sua razão de desempenho $r_{p, s}=r_{M}$, sendo $r_{M} \geq r_{p, s}$ para todo $p \in \mathcal{P}, s \in \mathcal{S}$. Ou seja, $r_{M}$ é a razão de desempenho máxima de um conjunto de problemas-teste P. Com isso, pode-se definir a função de distribuição acumulada da razão de desempenho $\rho_{s}(\tau)$, dada pela Equação B.2.

$$
\rho_{s}(\tau)=\frac{1}{n_{p}}\left|p \in \mathcal{P}: r_{p, s} \leq \tau\right|
$$

$\rho_{s}(\tau)$ pode ser visto como a probabilidade que a razão de desempenho $r_{p, s}$ do procedimento $s \in \mathcal{S}$ seja menor que fator $\tau$. Assim, a Equação B.2 pode ser reescrita como a Equação B.3. 


$$
\rho_{s}(\tau)=P\left(r_{p, s} \leq \tau: 1 \leq s \leq n_{s}\right)
$$

Para algumas comparações, por exemplo quando $\max \left\{r_{p, s}: s \in \mathcal{S}, p \in \mathcal{P}\right\}$ é muitas vezes maior que 1 (e consequentemente $r_{M}$ também é muitas vezes maior), a probabilidade $\rho_{s}(\tau)$ pode utilizar a escala logarítmica para facilitar a visualização.

O primeiro detalhe que o perfil de desempenho apresenta é com relação ao eixo vertical, quando o parâmetro $\tau$ é mínimo. Nele está a porcentagem das vezes que o procedimento apresenta o melhor desempenho entre os procedimentos concorrentes. Obviamente que quanto maior o "início" das curvas de desempenho, melhor o procedimento. Outro detalhe é que a medida que o valor de $\tau$ aumenta, as curvas de desempenho de cada procedimento tendem a crescer, dado que os procedimentos cujas curvas são mais inclinadas têm seu desempenho mais próximo do melhor desempenho. Por último, ao final do gráfico de perfil de desempenho a porcentagem de problemas-teste resolvidos pelo procedimento é apresentada. Assim, pode-se analisar capacidade do procedimento de se encontrar soluções factíveis, por exemplo. 


\section{APÊENDICE C - Resultados Gerais}

As tabelas de resultados das heurísticas da literatura e das metaheurísticas propostas foram divididas por classe de problemas-teste. As Tabelas 17-36 apresentam os resultados das heurística da literatura enquanto as Tabelas 37-56 apresentam os resultados das principais metaheurísticas propostas. Em todas tabelas, a primeira coluna define o código do problema-teste, dado por $\beta$ num, sendo $\beta$ a porcentagem da capacidade máxima utilizada e num a identificação do problema-teste. As colunas posteriores são relativas aos resultados das abordagens utilizadas, desde procedimentos exatos, cálculo de limitantes e resultados de heurísticas da literatura e das metaheurísticas propostas. O símbolo "-" é utilizado quando uma solução não é obtida para determinada abordagem.

Tabela 17: Problemas-teste da classe $(3,3,3)$.

\begin{tabular}{|c|ccccccc|}
\hline & MIP & LB1 & LB2 & RH1 & RH2 & RH3 & RF1 \\
\hline 060_000 & 5251,70 & 4410,95 & 5075,85 & - & 5257,09 & 5251,70 & 5251,70 \\
060_001 & 5340,88 & 4557,93 & 5036,94 & - & 5340,88 & - & - \\
060_002 & - & 5017,68 & 5655,10 & - & - & - & - \\
060_003 & 6115,74 & 5618,28 & 6108,80 & 6424,62 & 6115,74 & 6261,22 & 6261,22 \\
060_004 & 5288,71 & 4646,79 & 5135,07 & - & 5294,92 & 5438,10 & 5438,10 \\
070_000 & 4892,34 & 4360,38 & 4892,34 & 5081,15 & 4923,95 & 4892,34 & 4892,34 \\
070_001 & 5037,96 & 4527,47 & 4950,58 & 5342,51 & 5219,94 & 5181,11 & 5181,11 \\
070_002 & 5629,75 & 4969,73 & 5480,60 & 5791,42 & 5629,75 & 5782,63 & 5792,63 \\
070_003 & 6099,12 & 5561,06 & 6099,12 & 6251,13 & 6099,12 & 6245,48 & 6245,48 \\
070_004 & 5119,00 & 4613,02 & 5119,00 & 5125,06 & 5141,00 & 5119,00 & 5119,00 \\
080_000 & 4891,00 & 4332,20 & 4821,68 & 4910,48 & 4970,00 & 4891,00 & 4901,00 \\
080_001 & 4955,08 & 4509,60 & 4887,83 & 5032,32 & 5031,00 & 5172,90 & 5172,90 \\
080_002 & 5472,00 & 4949,75 & 5472,00 & 5632,84 & 5627,00 & 5627,00 & 5472,00 \\
080_003 & 6094,12 & 5524,26 & 6052,60 & 6246,13 & 6094,12 & 6240,48 & 6240,48 \\
080_004 & 5119,00 & 4588,57 & 5119,00 & 5119,00 & 5141,00 & 5119,00 & 5119,00 \\
\hline
\end{tabular}

Tabela 18: Problemas-teste da classe $(5,3,3)$.

\begin{tabular}{|l|ccccccc|}
\hline & MIP & LB1 & LB2 & RH1 & RH2 & RH3 & RF1 \\
\hline 060_005 & - & 7224,76 & 8297,10 & - & - & - & - \\
060_006 & 5839,00 & 5063,35 & 5772,96 & 6055,02 & 5860,00 & 6055,00 & 6300,53 \\
060_007 & 8854,73 & 7580,76 & 8679,02 & 9223,51 & 8854,73 & 9244,24 & 9334,42 \\
060_008 & 8474,46 & 7445,16 & 8155,46 & 8798,22 & 8573,68 & 8778,66 & 8887,31 \\
060_009 & 8635,24 & 7407,97 & 8435,34 & 8836,54 & 8667,48 & 8846,00 & 9051,00 \\
070_005 & 8256,20 & 7199,67 & 8146,34 & 8491,15 & 8351,19 & 8576,79 & 8720,38 \\
070_006 & 5762,00 & 5050,73 & 5732,28 & 6055,00 & 5860,00 & 6055,00 & 6292,00 \\
070_007 & 8649,90 & 7551,18 & 8494,25 & 9183,37 & 8799,78 & 9200,20 & 9284,10 \\
070_008 & 8346,15 & 7420,05 & 8144,00 & 8637,21 & 8356,53 & 8481,53 & 8609,77 \\
070_009 & 8431,00 & 7364,69 & 8339,20 & 8836,54 & 8554,00 & 8846,00 & 9051,00 \\
080_005 & 8146,00 & 7185,34 & 8022,99 & 8363,97 & 8146,00 & 8573,00 & 8397,09 \\
080_006 & 5749,00 & 5041,27 & 5715,87 & 6055,00 & 5775,00 & 6055,00 & 6292,00 \\
080_007 & 8539,20 & 7535,10 & 8394,54 & 9029,30 & 8682,36 & 9065,60 & 9096,16 \\
080_008 & 8239,00 & 7403,05 & 8142,14 & 8486,21 & 8376,00 & 8466,42 & 8474,00 \\
080_009 & 8405,00 & 7341,58 & 8288,38 & 8836,00 & 8512,00 & 8846,00 & 8944,00 \\
\hline
\end{tabular}


Tabela 19: Problemas-teste da classe $(3,5,3)$.

\begin{tabular}{|c|ccccccc|}
\hline & MIP & LB1 & LB2 & RH1 & RH2 & RH3 & RF1 \\
\hline 060_010 & 6913,60 & 6216,44 & 6868,14 & 7150,71 & 6913,60 & 7146,72 & 7403,97 \\
060_011 & - & - & - & - & - & - & - \\
060_012 & 6278,31 & 5518,50 & 6096,63 & 6783,01 & 6278,31 & 6517,63 & 6685,45 \\
060_013 & - & 6882,82 & 7708,36 & - & - & - & - \\
060_014 & 5897,00 & 5006,31 & 5894,48 & 6605,05 & 5897,00 & 6608,25 & 6327,25 \\
070_010 & 6872,22 & 6178,90 & 6826,75 & 7132,81 & 6872,22 & 7122,22 & 7127,22 \\
070_011 & - & 7890,28 & 8858,63 & - & - & - & - \\
070_012 & 6165,08 & 5490,02 & 6022,71 & 6513,37 & 6267,51 & 6494,14 & 6639,21 \\
070_013 & - & 6828,93 & 7608,00 & - & - & - & - \\
070_014 & 5876,38 & 4975,39 & 5863,02 & 6323,66 & 5876,38 & 6587,63 & 6572,63 \\
080_010 & 6854,54 & 6159,62 & 6822,00 & 7330,12 & 6926,91 & 7319,54 & 7109,54 \\
080_011 & 8818,00 & 7856,20 & 8763,94 & 9028,92 & 8884,24 & 9019,00 & 8884,24 \\
080_012 & 6016,73 & 5466,35 & 5967,27 & 6501,37 & 6016,73 & 6482,14 & 6624,86 \\
080_013 & 7659,87 & 6800,10 & 7492,35 & 7707,32 & 7706,97 & 7716,97 & 7659,87 \\
080_014 & 5844,25 & 4947,05 & 5774,83 & 6303,03 & 5855,75 & 6567,00 & 6552,00 \\
\hline
\end{tabular}

Tabela 20: Problemas-teste da classe $(3,3,5)$.

\begin{tabular}{|l|ccccccc|}
\hline & MIP & LB1 & LB2 & RH1 & RH2 & RH3 & RF1 \\
\hline 060_015 & 8653,17 & 7909,83 & 8609,22 & - & 8653,17 & 9130,70 & 9224,33 \\
060_016 & - & 6651,20 & 7355,80 & - & - & - & - \\
060_017 & - & 7428,63 & 8196,53 & - & - & - & - \\
060_018 & 5627,68 & 4978,63 & 5627,68 & 5783,00 & 5891,90 & 5903,00 & 5902,32 \\
060_019 & - & 7992,03 & 8816,62 & - & - & - & - \\
070_015 & 8591,91 & 7833,21 & 8546,88 & 8874,27 & 8591,91 & 9082,88 & 9136,85 \\
070_016 & 7333,24 & 6620,99 & 7281,42 & 7659,65 & 7333,93 & 7659,65 & 7695,60 \\
070_017 & 8199,78 & 7358,59 & 8124,85 & 8745,18 & 8310,06 & 8739,08 & 8432,82 \\
070_018 & 5623,00 & 4968,11 & 5623,00 & 5783,00 & 5676,88 & 5903,00 & 5888,79 \\
070_019 & 8751,42 & 7911,66 & 8728,80 & 9101,45 & 8751,42 & 9260,32 & 9156,94 \\
080_015 & 8531,31 & 7806,74 & 8503,73 & 8932,08 & 8666,08 & 8930,72 & 9058,21 \\
080_016 & 7263,24 & 6605,56 & 7262,81 & 7652,65 & 7263,24 & 7652,65 & 7580,65 \\
080_017 & 8086,33 & 7325,13 & 8064,21 & 8299,74 & 8086,33 & 8441,37 & 8409,37 \\
080_018 & 5623,00 & 4961,97 & 5623,00 & 5963,00 & 5665,85 & 5903,00 & 5884,92 \\
080_019 & 8703,53 & 7872,00 & 8685,38 & 8799,70 & 8703,53 & 8932,00 & 8932,00 \\
\hline
\end{tabular}

Tabela 21: Problemas-teste da classe $(5,5,5)$.

\begin{tabular}{|l|ccccccc|}
\hline & MIP & LB1 & LB2 & RH1 & RH2 & RH3 & RF1 \\
\hline 060_020 & - & 23457,00 & 26017,80 & - & - & - & - \\
060_021 & 18246,34 & 16075,70 & 17875,84 & 19374,33 & 18576,40 & 19149,50 & 19835,70 \\
$060 \_022$ & - & 20040,39 & 22131,52 & - & - & - & - \\
060_023 & 21963,66 & 18968,66 & 21201,40 & 22838,83 & 22292,47 & - & - \\
060_024 & - & 24470,47 & - & - & - & - & - \\
070_020 & 26273,94 & 23302,15 & 25666,94 & 27207,17 & 26525,59 & 27157,51 & 27501,05 \\
070_021 & 18106,52 & 15995,61 & 17735,82 & 19336,36 & 18367,12 & 19120,23 & 19527,96 \\
070_022 & 22248,36 & 19934,40 & 21984,19 & 23847,71 & 22834,43 & 24345,26 & 24169,83 \\
070_023 & 21302,47 & 18860,76 & 20933,23 & 22168,24 & 21582,69 & 22522,88 & 22391,51 \\
070_024 & 27621,08 & 24217,63 & 26826,41 & - & 28179,17 & 28784,15 & 28525,70 \\
080_020 & 25846,22 & 23207,19 & 25530,60 & 26923,85 & 26170,28 & 26844,34 & 27221,31 \\
080_021 & 17838,29 & 15960,55 & 17696,11 & 18654,00 & 17986,43 & 18884,00 & 19637,66 \\
080_022 & 22135,11 & 19857,36 & 21927,83 & 23829,64 & 22382,96 & 24344,00 & 23883,36 \\
080_023 & 20990,91 & 18804,78 & 20716,24 & 21970,87 & 21276,82 & 22299,37 & 22263,24 \\
$080 \_024$ & 27052,89 & 24111,02 & 26365,95 & 28596,19 & 27576,69 & 28656,15 & 28418,26 \\
\hline
\end{tabular}

Tabela 22: Problemas-teste da classe $(7,5,5)$.

\begin{tabular}{|c|ccccccc|}
\hline & MIP & LB1 & LB2 & RH1 & RH2 & RH3 & RF1 \\
\hline 060_025 & 29467,89 & 25602,43 & 28623,07 & 31526,44 & 29539,29 & 30948,24 & 31480,53 \\
060_026 & 31084,41 & 26910,32 & 30049,75 & 32924,96 & 31247,66 & 33199,57 & 33267,94 \\
060_027 & 29702,40 & 25490,70 & 28395,06 & 31619,64 & 29777,51 & 32226,48 & 32044,85 \\
060_028 & 31201,56 & 26863,00 & 29932,55 & 33113,40 & 31414,90 & 33506,14 & 34064,69 \\
060_029 & 32540,11 & 28007,55 & 31058,18 & 34171,51 & 32451,57 & - & 34493,31 \\
070_025 & 28986,80 & 25506,91 & 28373,44 & 31023,77 & 29091,84 & 30882,87 & 31170,84 \\
070_026 & 30517,03 & 26796,84 & 29857,93 & 32913,62 & 30766,42 & 33168,80 & 33421,92 \\
070_027 & 28757,02 & 25394,94 & 28176,27 & 31321,65 & 29273,14 & 31564,69 & 32090,01 \\
070_028 & 30447,63 & 26757,60 & 29797,81 & 32879,55 & 31108,98 & 32891,31 & 33346,45 \\
070_029 & 31547,92 & 27874,68 & 30503,46 & 33905,84 & 31726,34 & 34003,27 & 34431,30 \\
080_025 & 28652,47 & 25440,25 & 28287,09 & 30622,48 & 29387,43 & 30691,00 & 30531,95 \\
080_026 & 30099,22 & 26720,44 & 29723,99 & 32861,18 & 30686,88 & 33149,02 & 33382,56 \\
080_027 & 28489,96 & 25345,86 & 27993,23 & 30836,33 & 29087,21 & 31168,00 & 31289,11 \\
080_028 & 29997,91 & 26685,00 & 29689,70 & 32348,08 & 30122,60 & 32618,64 & 33549,96 \\
080_029 & 30979,55 & 27784,73 & 30328,85 & 33041,23 & 31392,83 & 33703,32 & 34280,33 \\
\hline
\end{tabular}

Tabela 23: Problemas-teste da classe $(5,7,5)$.

\begin{tabular}{|l|ccccccc|}
\hline & MIP & LB1 & LB2 & RH1 & RH2 & RH3 & RF1 \\
\hline 060_030 & - & 30588,42 & 34164,86 & - & - & - & - \\
060_031 & - & 29742,57 & 33343,98 & - & - & - & - \\
060_032 & - & 20315,88 & 22790,86 & - & - & - & - \\
060_033 & - & 25404,74 & 28510,82 & - & - & - & - \\
060_034 & - & 32602,94 & 37003,68 & - & - & - & - \\
070_030 & 35009,10 & 30262,36 & 33258,86 & 37043,31 & 35072,62 & 37042,17 & 37113,22 \\
070_031 & - & 29519,66 & 32546,41 & - & - & - & - \\
070_032 & 22958,25 & 20188,17 & 22596,45 & 24920,03 & 24081,35 & 24803,07 & 25647,78 \\
070_033 & - & 25209,94 & 28025,36 & - & - & - & - \\
070_034 & - & 32350,67 & 35538,59 & - & - & - & - \\
080_030 & 33829,60 & 30051,41 & 32789,67 & 35673,97 & 34303,77 & 36500,34 & 36575,75 \\
080_031 & 33085,96 & 29390,56 & 32293,11 & 35272,74 & 33685,27 & 35570,85 & 35253,47 \\
080_032 & 22748,98 & 20133,43 & 22369,00 & 24210,56 & 23164,11 & 24747,14 & 25554,43 \\
080_033 & 28329,78 & 25118,31 & 27875,12 & 30899,08 & 28884,94 & 30880,04 & 30908,27 \\
080_034 & 36512,54 & 32216,08 & 35243,14 & 38264,92 & 36619,24 & 38469,57 & 38849,53 \\
\hline
\end{tabular}


Tabela 24: Problemas-teste da classe $(5,5,7)$.

\begin{tabular}{|c|ccccccc|}
\hline & MIP & LB1 & LB2 & RH1 & RH2 & RH3 & RF1 \\
\hline 060_035 & 32148,29 & 28657,54 & 31101,46 & 34472,87 & 32486,50 & 34654,63 & 34319,72 \\
060_036 & 32312,73 & 28223,23 & 30973,36 & - & 32486,66 & 34216,73 & 34408,10 \\
060_037 & 30804,71 & 27099,34 & 29466,36 & 32893,44 & 31102,58 & - & - \\
060_038 & 30963,80 & 27304,76 & 30063,72 & 33797,29 & 31414,49 & 34014,42 & 33899,38 \\
060_039 & 29439,79 & 25940,33 & 28421,80 & - & 29893,55 & 31572,72 & 31179,71 \\
070_035 & 31537,61 & 28497,15 & 30763,51 & 33842,66 & 31961,19 & 33195,88 & 33378,66 \\
070_036 & 31619,41 & 28059,81 & 30664,78 & 33722,36 & 31922,33 & 33901,82 & 33964,03 \\
070_037 & 30059,45 & 26944,56 & 29256,87 & 32710,56 & 30261,50 & 32986,14 & 32528,64 \\
070_038 & 30333,36 & 27160,28 & 29917,01 & 32704,97 & 30827,41 & 32971,58 & 33517,97 \\
070_039 & 28871,75 & 25818,12 & 28216,56 & 30529,88 & 29087,36 & 30961,31 & 31088,61 \\
080_035 & 31031,29 & 28395,62 & 30620,31 & 33202,54 & 31644,86 & 32783,04 & 32577,80 \\
080_036 & 31136,41 & 27958,70 & 30565,27 & 32962,84 & 31581,49 & 33603,23 & 33633,29 \\
080_037 & 29628,99 & 26835,48 & 29100,01 & 32442,44 & 29703,49 & 32676,45 & 32703,32 \\
080_038 & 30055,66 & 27077,15 & 29763,76 & 32724,04 & 30797,91 & 32937,42 & 33488,10 \\
080_039 & 28505,06 & 25736,97 & 28045,41 & 30431,44 & 29192,81 & 30919,43 & 30834,32 \\
\hline
\end{tabular}

Tabela 25: Problemas-teste da classe $(7,7,7)$.

\begin{tabular}{|l|ccccccc|}
\hline & MIP & LB1 & LB2 & RH1 & RH2 & RH3 & RF1 \\
\hline 060_040 & 61817,64 & 51563,90 & - & 63154,09 & 59192,00 & 63222,97 & 63814,84 \\
$060 \_041$ & - & 54615,31 & - & - & - & - & - \\
$060 \_042$ & - & 46955,38 & - & 58807,68 & 54975,25 & 59036,09 & 59472,79 \\
$060 \_043$ & - & 52135,93 & 57927,31 & - & - & - & - \\
$060 \_044$ & - & 53862,62 & - & - & 62575,71 & 65712,45 & 67015,42 \\
$070 \_040$ & 58722,98 & 51348,82 & - & 62435,78 & 57711,74 & 62686,60 & 63230,25 \\
$070 \_041$ & - & 54402,89 & - & 65482,40 & 61886,28 & 65514,62 & 66119,04 \\
$070 \_042$ & 55876,88 & 46728,29 & - & 57225,56 & 54376,68 & 58787,72 & 58525,94 \\
$070 \_043$ & - & 51934,26 & - & - & - & - & - \\
$070 \_044$ & - & 53606,33 & - & 65459,65 & 61832,07 & 65470,33 & 66312,51 \\
$080 \_040$ & 57832,77 & 51206,16 & - & 60921,72 & 57738,46 & 62285,17 & 62357,58 \\
$080 \_041$ & 63899,88 & 54244,86 & - & 65037,28 & 60869,02 & 65074,65 & 65975,81 \\
$080 \_042$ & 53614,13 & 46559,74 & - & 56679,44 & 53229,45 & 58275,54 & 58052,18 \\
$080 \_043$ & 58474,61 & 51792,53 & 56620,30 & 64335,02 & 59189,23 & 64226,27 & 64499,18 \\
$080 \_044$ & - & 53449,75 & 58046,24 & 64072,14 & 59862,32 & 65053,54 & 66525,11 \\
\hline
\end{tabular}

Tabela 26: Problemas-teste da classe $(10,5,5)$.

\begin{tabular}{|l|ccccccc|}
\hline & MIP & LB1 & LB2 & RH1 & RH2 & RH3 & RF1 \\
\hline 060_045 & 51790,92 & 41231,39 & 45959,33 & 49650,13 & 47998,84 & 50017,99 & 51020,08 \\
060_046 & - & 43683,55 & 48739,02 & 52419,02 & 51288,17 & 53256,31 & 53228,90 \\
060_047 & - & 40221,41 & 45235,19 & - & - & - & - \\
060_048 & - & 39591,90 & 44686,67 & 48624,73 & 46140,36 & 49015,38 & 49201,35 \\
060_049 & - & 43285,55 & 48829,74 & 52604,83 & 51596,75 & 53226,65 & 53838,12 \\
070_045 & - & 41108,31 & 45839,92 & 49097,00 & 46945,52 & 49017,43 & 50417,56 \\
070_046 & - & 43521,94 & 48182,73 & 51501,15 & 49996,82 & 51773,59 & 51797,29 \\
070_047 & - & 40064,95 & 44517,26 & - & 46161,83 & 49499,74 & 49235,75 \\
070_048 & - & 39459,48 & - & 48046,34 & 45081,34 & 48651,57 & 48408,31 \\
070_049 & 50781,04 & 43114,02 & 48011,32 & - & - & 52646,97 & 53172,17 \\
080045 & - & 41020,74 & 45772,50 & 48876,34 & 46410,33 & 48944,75 & 49867,82 \\
080046 & 51014,59 & 43427,23 & 47935,40 & 51261,28 & 49432,38 & 51006,68 & 51082,25 \\
080_047 & 48909,31 & 39970,13 & - & 48006,26 & 46107,39 & 48558,21 & 48274,89 \\
080_048 & 45634,67 & 39371,39 & 44053,85 & 47757,35 & 44903,29 & 48611,00 & 48753,13 \\
080_049 & - & 42996,47 & 47762,31 & 51696,62 & 49321,21 & 51782,29 & 52315,28 \\
\hline
\end{tabular}

Tabela 27: Problemas-teste da classe $(5,10,5)$.

\begin{tabular}{|c|ccccccc|}
\hline & MIP & LB1 & LB2 & RH1 & RH2 & RH3 & RF1 \\
\hline 060_050 & - & 49028,64 & 54915,90 & - & - & - & - \\
060_051 & 49327,37 & 41415,71 & 45565,34 & 51165,21 & 48687,93 & 50662,36 & 50845,71 \\
060_052 & 34054,80 & 29384,99 & 33122,34 & - & 34258,35 & 36851,96 & 36495,95 \\
060_053 & - & 35773,65 & 40002,90 & - & - & - & - \\
060_054 & - & 32583,78 & 36243,18 & - & - & - & - \\
070_050 & - & 48625,07 & 53255,50 & - & - & - & - \\
070_051 & 47065,36 & 41139,40 & 45113,09 & 50550,34 & 47828,33 & 50377,28 & 50142,95 \\
070_052 & 33328,72 & 29230,36 & 32625,16 & 35995,60 & 33757,12 & 36721,78 & 36467,70 \\
070_053 & 40460,42 & 35596,84 & 39067,06 & 43758,52 & 41379,88 & 43190,26 & 43337,85 \\
070_054 & 36945,27 & 32391,01 & 35949,58 & 39593,10 & 37165,62 & 41539,77 & 41066,05 \\
080_050 & 54514,10 & 48411,23 & 52602,56 & 55872,26 & 54834,53 & 56881,88 & 57011,97 \\
080_051 & 46136,83 & 40984,54 & 44853,48 & 49802,67 & 46860,41 & 49266,04 & 50122,11 \\
080_052 & 33011,02 & 29122,45 & 32319,20 & 35400,18 & 33649,17 & 36189,71 & 36343,17 \\
080_053 & 39814,90 & 35485,36 & 38583,30 & 43681,02 & 41017,38 & 43092,92 & 43936,40 \\
080_054 & 36710,42 & 32261,81 & 35748,14 & 39614,31 & 37105,00 & 41466,44 & 41017,88 \\
\hline
\end{tabular}

Tabela 28: Problemas-teste da classe $(5,5,10)$.

\begin{tabular}{|l|ccccccc|}
\hline & MIP & LB1 & LB2 & RH1 & RH2 & RH3 & RF1 \\
\hline 060_055 & 43458,33 & 38557,35 & - & - & 43929,42 & 46532,67 & 46565,45 \\
060_056 & 46693,99 & 41435,91 & - & - & 46972,82 & 49511,98 & 49439,09 \\
060_057 & 42405,91 & 36843,40 & 40452,80 & - & 42894,88 & 45143,68 & 45133,54 \\
060_058 & - & 39489,15 & - & - & - & - & - \\
060_059 & 49060,34 & 43252,06 & - & - & 49233,39 & - & - \\
070_055 & 43057,29 & 38327,15 & 41547,90 & 46327,72 & 43037,47 & 46655,31 & 46366,70 \\
070_056 & 45727,15 & 41175,35 & 44575,33 & 48952,20 & 45995,78 & 48577,49 & 48890,76 \\
070_057 & 41282,58 & 36589,87 & 40027,56 & 44442,23 & 42142,88 & 44958,60 & 45012,75 \\
070_058 & 44078,32 & 39232,74 & - & - & 44364,08 & 47307,06 & 47645,32 \\
070_059 & 47960,91 & 42976,92 & 46147,77 & 50654,95 & 48124,23 & 50424,79 & 51248,63 \\
080_055 & 42110,97 & 38173,16 & 41345,31 & 45436,98 & 42354,25 & 45474,05 & 45928,04 \\
080_056 & 45032,21 & 41005,11 & 44353,92 & 48429,45 & 45703,03 & 48095,53 & 48420,72 \\
080_057 & 40914,61 & 36449,55 & 39856,62 & 44166,20 & 41771,98 & 44850,61 & 44868,64 \\
080_058 & 43384,27 & 39068,36 & 42414,33 & 46583,57 & 43737,21 & 46726,69 & 47115,22 \\
080_059 & 47351,04 & 42790,22 & 46011,05 & 49689,30 & 47249,51 & 50105,42 & 50814,94 \\
\hline
\end{tabular}


Tabela 29: Problemas-teste da classe $(10,7,7)$.

\begin{tabular}{|c|ccccccc|}
\hline & MIP & LB1 & LB2 & RH1 & RH2 & RH3 & RF1 \\
\hline 060_060 & - & 79281,88 & - & - & - & - & - \\
060_061 & - & 71913,34 & - & - & - & - & - \\
060_062 & - & 71184,48 & - & - & - & 90814,21 & 89865,82 \\
060_063 & - & 77070,64 & - & - & - & - & - \\
060_064 & - & 80763,67 & - & - & - & - & 100853,47 \\
070_060 & - & 78933,47 & - & - & - & 97722,24 & 97665,94 \\
070_061 & - & 71630,41 & - & - & - & 91123,93 & 91418,26 \\
070_062 & - & 70970,97 & - & 88186,97 & 81788,92 & 88018,90 & 88591,06 \\
070_063 & - & 76654,09 & - & 96376,38 & - & 95860,88 & - \\
070_064 & - & 80484,57 & - & 99373,70 & 93220,86 & 99344,63 & 100445,06 \\
080_060 & - & 78713,43 & - & - & 90121,17 & 97053,61 & 97345,07 \\
080_061 & - & 71438,77 & - & 89431,42 & 83130,93 & 90315,95 & 90420,67 \\
080_062 & - & 70821,50 & - & - & - & 87419,76 & 88082,55 \\
080_063 & - & 76403,10 & - & - & - & 95078,79 & 94223,86 \\
080_064 & - & 80287,12 & - & - & - & 98835,36 & 98417,95 \\
\hline
\end{tabular}

Tabela 30: Problemas-teste da classe $(7,10,7)$.

\begin{tabular}{|c|ccccccc|}
\hline & MIP & LB1 & LB2 & RH1 & RH2 & RH3 & RF1 \\
\hline 060_065 & - & 74372,11 & - & - & - & 95028,28 & - \\
060_066 & - & 76604,67 & - & - & - & - & - \\
060_067 & 85761,68 & 66507,67 & - & - & 78857,50 & - & 84097,16 \\
060_068 & - & 77318,02 & - & - & - & - & - \\
060_069 & - & 86071,91 & - & - & - & - & - \\
070_065 & - & 73888,86 & - & 91245,04 & 86752,10 & 93490,68 & 92901,42 \\
070_066 & - & 76122,57 & - & 95782,44 & 90408,82 & 94804,42 & 95147,34 \\
070_067 & - & 66256,41 & - & 83340,07 & 76995,34 & 82963,21 & 82090,10 \\
070_068 & - & 76968,08 & - & 93819,23 & 89631,08 & 95285,90 & 95190,70 \\
070_069 & - & 85546,44 & - & - & - & - & - \\
080_065 & - & 73558,75 & - & - & 84163,07 & 91749,67 & 92530,32 \\
080_066 & - & 75847,53 & - & - & 88364,80 & 94481,54 & 94207,18 \\
080_067 & - & 66089,90 & - & 81330,95 & 75420,26 & 82283,96 & 82740,37 \\
080_068 & 87671,18 & 76720,85 & - & 92960,89 & 87327,17 & 95085,73 & 94393,66 \\
080_069 & - & 85204,08 & - & 100980,27 & 97673,94 & 103184,75 & 100198,49 \\
\hline
\end{tabular}

Tabela 31: Problemas-teste da classe $(7,7,10)$.

\begin{tabular}{|l|ccccccc|}
\hline & MIP & LB1 & LB2 & RH1 & RH2 & RH3 & RF1 \\
\hline 060_070 & - & 80626,84 & - & - & - & - & - \\
060_071 & - & 69186,39 & - & 85918,31 & 80031,03 & 85801,32 & 85350,66 \\
060_072 & - & 79229,44 & - & - & 91484,63 & 98306,60 & 98761,85 \\
060_073 & - & 81102,75 & - & - & - & - & - \\
060_074 & - & 80199,32 & - & - & 92985,96 & - & - \\
070_070 & - & 80174,90 & - & 98624,94 & 91378,83 & 98143,35 & 98708,48 \\
070_071 & - & 68878,22 & - & 84334,77 & 79008,45 & 84793,92 & 85460,89 \\
070_072 & - & 78856,84 & - & - & 89660,03 & 97694,40 & 97673,95 \\
070_073 & - & 80639,57 & - & 96743,32 & 91882,83 & - & 99752,58 \\
070_074 & - & 79801,43 & - & 97274,27 & 90684,42 & 98006,76 & 98653,51 \\
080_070 & - & 79859,28 & - & 97187,75 & 91077,83 & 96940,51 & 97632,36 \\
080_071 & - & 68692,76 & - & 83162,87 & 77470,98 & 84044,46 & 84516,98 \\
080_072 & 91582,87 & 78599,68 & - & 95161,34 & 88337,54 & 97190,88 & 97551,11 \\
080_073 & - & 80303,40 & - & 95521,14 & 91163,76 & 98853,01 & 97975,18 \\
080_074 & - & 79509,41 & - & 95833,81 & 89410,57 & 97133,62 & 97602,07 \\
\hline
\end{tabular}

Tabela 32: Problemas-teste da classe $(10,10,10)$.

\begin{tabular}{|l|ccccccc|}
\hline & MIP & LB1 & LB2 & RH1 & RH2 & RH3 & RF1 \\
\hline $060 \_075$ & - & 146028,23 & - & - & - & - & - \\
$060 \_076$ & - & 151020,34 & - & - & - & - & - \\
$060 \_077$ & - & 166593,44 & - & - & - & - & - \\
$060 \_078$ & - & 175942,64 & - & - & - & - & - \\
$060 \_079$ & - & 166936,77 & - & - & - & - & - \\
$070 \_075$ & - & 145520,82 & - & - & - & 181440,92 & - \\
$070 \_076$ & - & 150469,95 & - & - & - & 192865,35 & - \\
$070 \_077$ & - & 165767,85 & - & - & - & - & - \\
$070 \_078$ & - & 175116,39 & - & - & - & 217331,34 & - \\
$070 \_079$ & - & 166376,79 & - & - & - & 206680,01 & - \\
$080 \_075$ & - & 145182,16 & - & - & - & 180119,79 & - \\
$080 \_076$ & - & 150096,96 & - & - & - & 190684,93 & 191452,31 \\
$080 \_077$ & - & 165194,62 & - & 211513,99 & - & 201700,56 & - \\
$080 \_078$ & - & 174579,96 & - & - & - & 214362,19 & - \\
$080 \_079$ & - & 165976,04 & - & - & - & 204992,70 & 205303,58 \\
\hline
\end{tabular}

Tabela 33: Problemas-teste da classe $(15,10,10)$.

\begin{tabular}{|l|ccccccc|}
\hline & MIP & LB1 & LB2 & RH1 & RH2 & RH3 & RF1 \\
\hline 060_080 & - & 246526,79 & - & - & - & 309241,28 & - \\
060_081 & - & 227968,98 & - & - & - & 290872,54 & - \\
060_082 & - & 247479,14 & - & - & - & 311117,33 & - \\
060_083 & - & 202790,72 & - & - & - & 261156,86 & - \\
060_084 & - & 239026,58 & - & - & - & 300505,06 & - \\
070_080 & - & 245825,59 & - & - & - & 306655,83 & - \\
070_081 & - & 227392,97 & - & - & - & 286342,55 & - \\
070_082 & - & 246851,35 & - & - & - & 308752,57 & - \\
070_083 & - & 202220,95 & - & - & - & 257792,97 & - \\
070_084 & - & 238384,88 & - & - & - & 296177,78 & - \\
080_080 & - & 245373,47 & - & - & - & 301919,17 & - \\
080_081 & - & 226975,68 & - & - & - & 284902,60 & - \\
080_082 & - & 246418,09 & - & - & - & 306024,59 & - \\
0800083 & - & 201818,81 & - & - & - & 256894,11 & - \\
080_084 & - & 237948,47 & - & - & - & 293546,25 & - \\
\hline
\end{tabular}


Tabela 34: Problemas-teste da classe $(10,15,10)$.

\begin{tabular}{|c|c|c|c|c|c|c|c|}
\hline & MIF & LB1 & LB2 & RH1 & $\mathrm{RH} 2$ & RH3 & RF1 \\
\hline 060_085 & - & 232317,11 & - & - & - & - & \\
\hline 060_086 & - & 234591,45 & - & - & - & - & - \\
\hline 060_087 & - & 257840,18 & - & - & - & - & - \\
\hline 060_088 & - & 243604,53 & - & - & - & - & - \\
\hline 060_089 & - & 249144,21 & - & - & - & - & - \\
\hline 070_085 & - & 231101,88 & - & - & - & & - \\
\hline 070_086 & - & 233490,25 & - & - & - & 292461,29 & \\
\hline 070_087 & - & 256719,34 & - & - & - & - & - \\
\hline 070_088 & - & 242574,80 & - & - & - & - & - \\
\hline 070_089 & - & 247803,53 & - & - & - & 311014,90 & - \\
\hline 080_085 & - & 230411,01 & - & - & - & 288893,21 & - \\
\hline 080_086 & - & 232704,69 & - & - & - & 291446,41 & - \\
\hline 080_087 & - & 256070,69 & - & - & - & 315132,79 & - \\
\hline 080_088 & - & 241877,98 & - & - & - & 299341,69 & - \\
\hline 080_089 & - & 246891,28 & - & - & - & 307823,69 & - \\
\hline
\end{tabular}

Tabela 35: Problemas-teste da classe $(10,10,15)$.

\begin{tabular}{|c|ccccccc|}
\hline & MIP & LB1 & LB2 & RH1 & RH2 & RH3 & RF1 \\
\hline 060_090 & - & 256460,30 & - & - & - & - & - \\
060_091 & - & 225559,97 & - & - & - & - & - \\
060_092 & - & 258993,41 & - & - & - & - & - \\
060_093 & - & 226217,44 & - & - & - & - & - \\
060_094 & - & 239995,80 & - & - & - & 301650,65 & - \\
070_090 & - & 255240,91 & - & - & - & 315168,45 & - \\
070_091 & - & 224769,28 & - & - & - & 283373,17 & - \\
070_092 & - & 257940,87 & - & - & - & 317176,51 & - \\
070_093 & - & 225382,30 & - & - & - & 282971,08 & - \\
070_094 & - & 239194,02 & - & - & - & 298429,08 & - \\
080_090 & - & 254468,71 & - & - & - & 312242,72 & - \\
080_091 & - & 224191,92 & - & - & - & 280131,97 & - \\
080_092 & - & 257223,42 & - & - & - & 314408,95 & - \\
080_093 & - & 224796,90 & - & - & - & 281817,73 & - \\
080_094 & - & 238621,44 & - & - & - & 296173,60 & - \\
\hline
\end{tabular}

Tabela 36: Problemas-teste da classe $(15,15,15)$.

\begin{tabular}{|c|c|c|c|c|c|c|c|}
\hline & MIP & LB1 & LB2 & RH1 & RH2 & RH3 & RF1 \\
\hline $060 \_095$ & - & 514191,54 & - & - & - & - & - \\
\hline 060_096 & - & - & - & - & - & - & - \\
\hline 060_097 & - & - & - & - & - & - & - \\
\hline 060_098 & - & - & - & - & - & - & - \\
\hline 060_099 & - & - & - & - & - & - & - \\
\hline 070_095 & - & 512517,52 & - & - & - & - & - \\
\hline 070_096 & - & 528497,33 & - & - & - & - & - \\
\hline 070_097 & - & 537031,99 & - & - & - & - & - \\
\hline 070_098 & - & 508313,32 & - & - & - & - & - \\
\hline 070_099 & - & 540678,11 & - & - & - & - & - \\
\hline 080_095 & - & 511378,82 & - & - & - & 649163,25 & - \\
\hline 080_096 & - & 527131,49 & - & - & - & 666285,78 & - \\
\hline 080_097 & - & 535857,98 & - & - & - & - & - \\
\hline 080_098 & - & 507275,86 & - & - & - & 645142,43 & - \\
\hline 080_099 & - & 539469,96 & - & - & - & - & - \\
\hline
\end{tabular}

Tabela 37: Problemas-teste da classe $(3,3,3)$.

\begin{tabular}{|c|cccccccc|}
\hline & AT1 & AT2 & AT3 & AT4 & AT5 & AT6 & ATH1 & ATH2 \\
\hline 060_000 & 5258,80 & 5258,80 & 5258,80 & 5258,80 & 5258,80 & 5253,47 & 5251,70 & 5251,70 \\
060_001 & 5346,50 & 5346,50 & - & - & 5343,50 & 5346,50 & 5340,88 & 5340,88 \\
060_002 & - & - & - & - & - & - & - & - \\
060_003 & 6281,69 & 6490,06 & 6302,41 & 6301,83 & 6275,92 & 6476,44 & 6271,90 & 6271,90 \\
060_004 & 5301,61 & 5440,30 & 5293,89 & 5300,31 & 5293,71 & 5297,14 & 5438,10 & 5438,10 \\
070_000 & 4892,34 & 4910,53 & 4932,21 & 5252,80 & 4892,34 & 4892,34 & 4895,06 & 4895,06 \\
070_001 & 5037,96 & 5037,96 & 5037,96 & 5322,18 & 5037,96 & 5037,96 & 5037,96 & 5037,96 \\
070_002 & 5654,95 & 5644,73 & 5644,21 & 5644,21 & 5644,21 & 5644,21 & 5642,52 & 5642,52 \\
070_003 & 6099,12 & 6245,49 & 6113,00 & 6448,01 & 6099,12 & 6099,12 & 6245,49 & 6245,49 \\
070_004 & 5119,00 & 5272,91 & 5119,00 & 5119,00 & 5119,00 & 5129,28 & 5119,00 & 5119,00 \\
080_000 & 4891,00 & 4891,00 & 4915,00 & 4916,93 & 4891,00 & 4891,00 & 4891,00 & 4891,00 \\
080_001 & 4955,08 & 5031,00 & 4955,08 & 4955,08 & 4955,08 & 4955,08 & 4955,08 & 4955,08 \\
080_002 & 5472,00 & 5477,00 & 5477,00 & 5477,00 & 5472,00 & 5472,00 & 5472,00 & 5472,00 \\
080_003 & 6094,12 & 6094,12 & 6113,00 & 6113,00 & 6094,12 & 6094,12 & 6113,00 & 6100,00 \\
080_004 & 5119,00 & 5266,00 & 5119,00 & 5119,00 & 5119,00 & 5119,00 & 5119,00 & 5119,00 \\
\hline
\end{tabular}

Tabela 38: Problemas-teste da classe $(5,3,3)$.

\begin{tabular}{|l|cccccccc|}
\hline & AT1 & AT2 & AT3 & AT4 & AT5 & AT6 & ATH1 & ATH2 \\
\hline 060_005 & - & - & - & - & - & - & - & - \\
060_006 & 5868,67 & 5998,00 & 5860,00 & 5860,00 & 5872,00 & 5860,00 & 5860,00 & 5860,00 \\
060_007 & 9224,24 & 9224,24 & 9022,83 & 9221,67 & 9021,83 & 9224,24 & 9220,07 & 9022,83 \\
060_008 & 8509,50 & 8764,82 & 8509,50 & 8588,40 & 8509,50 & 8718,64 & 8764,82 & 8764,82 \\
060_009 & 8750,00 & 8836,00 & 8690,53 & 8843,00 & 8750,00 & 8750,00 & 8750,00 & 8750,00 \\
070_005 & 8368,56 & 8563,00 & 8351,40 & 8372,65 & 8288,25 & 8402,65 & 8372,65 & 8368,72 \\
070_006 & 5834,00 & 5977,00 & 5762,00 & 5762,00 & 5834,00 & 5762,00 & 5833,00 & 5762,00 \\
070_007 & 8649,90 & 8801,00 & 8649,90 & 8801,00 & 8649,90 & 8649,90 & 8702,00 & 8693,50 \\
070_008 & 8474,88 & 8474,88 & 8474,88 & 8474,88 & 8474,88 & 8523,00 & 8474,88 & 8474,88 \\
070_009 & 8561,00 & 8736,00 & 8561,00 & 8843,00 & 8561,00 & 8590,00 & 8561,00 & 8561,00 \\
080_005 & 8216,98 & 8408,00 & 8146,00 & 8300,00 & 8216,98 & 8170,42 & 8197,00 & 8158,78 \\
080_006 & 5749,00 & 5977,00 & 5749,00 & 5749,00 & 5749,00 & 5762,00 & 5749,00 & 5749,00 \\
080_007 & 8588,00 & 8774,00 & 8539,20 & 8702,00 & 8539,20 & 8678,00 & 8621,20 & 8588,00 \\
080_008 & 8239,00 & 8321,00 & 8239,00 & 8239,00 & 8239,00 & 8239,00 & 8239,00 & 8239,00 \\
080_009 & 8561,00 & 8662,00 & 8561,00 & 8843,00 & 8469,00 & 8578,00 & 8564,00 & 8523,00 \\
\hline
\end{tabular}


Tabela 39: Problemas-teste da classe $(3,5,3)$.

\begin{tabular}{|c|cccccccc|}
\hline & AT1 & AT2 & AT3 & AT4 & AT5 & AT6 & ATH1 & ATH2 \\
\hline 060_010 & 7156,23 & 7358,88 & 7161,59 & 7161,59 & 7146,72 & 7146,72 & 7146,72 & 7146,72 \\
060_011 & - & - & - & - & - & - & - & - \\
060_012 & 6541,75 & 6776,34 & 6454,16 & 6459,73 & 6493,58 & 6515,50 & 6538,22 & 6472,67 \\
060_013 & - & - & - & - & - & - & - & - \\
060_014 & 6072,25 & 6328,25 & 6106,68 & 6365,68 & 6072,25 & 6072,25 & 6072,25 & 6072,25 \\
070_010 & 6872,22 & 7127,22 & 6878,53 & 6878,53 & 6872,22 & 6872,22 & 6872,22 & 6872,22 \\
070_011 & - & - & - & - & - & - & - & - \\
070_012 & 6165,08 & 6322,00 & 6165,08 & 6165,08 & 6239,14 & 6239,14 & 6494,14 & 6494,14 \\
070_013 & - & - & - & - & - & - & - & - \\
070_014 & 6051,63 & 6069,13 & 5885,00 & 6359,00 & 6051,63 & 6051,63 & 6051,63 & 5885,00 \\
080_010 & 6854,54 & 7109,54 & 6862,47 & 6862,47 & 6854,54 & 6854,54 & 6854,54 & 6854,54 \\
080_011 & 8818,00 & 8818,00 & 8818,00 & 8818,00 & 8818,00 & 8818,00 & 8818,00 & 8818,00 \\
080_012 & 6227,14 & 6236,87 & 6027,94 & 6027,94 & 6027,94 & 6227,14 & 6482,14 & 6482,14 \\
080_013 & 7660,51 & 7660,75 & 7683,00 & 7683,00 & 7662,53 & 7661,13 & 7672,02 & 7672,02 \\
080_014 & 6031,00 & 6048,50 & 5885,00 & 6359,00 & 5848,25 & 5844,25 & 5885,00 & 5885,00 \\
\hline
\end{tabular}

Tabela 40: Problemas-teste da classe $(3,3,5)$.

\begin{tabular}{|l|cccccccc|}
\hline & AT1 & AT2 & AT3 & AT4 & AT5 & AT6 & ATH1 & ATH2 \\
\hline 060_015 & 9038,38 & 8971,11 & 8876,05 & 9009,67 & 9091,38 & 9020,77 & 8919,79 & 8919,79 \\
060_016 & - & - & - & - & - & - & - & - \\
060_017 & - & - & - & - & - & - & - & - \\
060_018 & 5707,94 & 5843,20 & 5737,42 & 5737,42 & 5631,00 & 5689,00 & 5631,00 & 5631,00 \\
060_019 & - & - & - & - & - & - & - & - \\
070_015 & 8591,91 & 8870,74 & 8604,56 & 8604,56 & 8735,88 & 8594,85 & 8755,44 & 8755,15 \\
070_016 & 7597,00 & 7669,65 & 7561,07 & 7594,00 & 7552,88 & 7597,00 & 7598,00 & 7598,00 \\
070_017 & 8306,95 & 8361,95 & 8365,21 & 8479,73 & 8468,95 & 8307,00 & 8367,95 & 8341,21 \\
070_018 & 5631,00 & 5783,00 & 5679,83 & 5679,83 & 5631,00 & 5631,00 & 5631,00 & 5631,00 \\
070_019 & 9104,89 & 8960,15 & 8991,02 & 9104,89 & 8852,39 & 9025,89 & 9090,24 & 9090,24 \\
080_015 & 8571,31 & 8850,87 & 8596,52 & 8596,52 & 8561,72 & 8578,52 & 8578,52 & 8578,52 \\
080_016 & 7413,00 & 7561,00 & 7413,00 & 7413,00 & 7413,00 & 7425,00 & 7322,00 & 7413,00 \\
080_017 & 8142,80 & 8231,80 & 8142,80 & 8142,80 & 8142,80 & 8217,32 & 8233,37 & 8142,80 \\
080_018 & 5631,00 & 5709,00 & 5659,00 & 5659,00 & 5631,00 & 5631,00 & 5631,00 & 5631,00 \\
080_019 & 8828,00 & 8828,00 & 8725,00 & 8912,00 & 8718,45 & 8819,00 & 8828,00 & 8725,00 \\
\hline
\end{tabular}

Tabela 41: Problemas-teste da classe $(5,5,5)$.

\begin{tabular}{|c|c|c|c|c|c|c|c|c|}
\hline & AT1 & AT2 & AT3 & AT4 & AT5 & AT6 & ATH1 & ATH2 \\
\hline $\begin{array}{l}060 \_020 \\
\end{array}$ & - & - & - & - & - & - & - & - \\
\hline 060_021 & 18938,04 & 19328,69 & 18599,08 & 18999,08 & 18816,84 & 18786,08 & 18874,92 & 18749,13 \\
\hline 060_022 & - & - & - & - & - & - & - & - \\
\hline 060_023 & 22742,21 & 22852,94 & - & - & 22663,88 & 22570,74 & 22678,27 & 22678,91 \\
\hline 060_024 & - & - & - & - & - & - & - & - \\
\hline 070_020 & 26966,94 & 27684,55 & 26754,13 & 26730,90 & 26737,20 & 27140,74 & 27408,84 & 27232,75 \\
\hline 070_021 & 18512,00 & 19164,00 & 18260,28 & 18694,04 & 18331,04 & 18448,17 & 18689,74 & 18344,98 \\
\hline 070_022 & 23214,58 & 23724,00 & 22824,68 & 23975,84 & 23495,46 & 23361,61 & 23059,00 & 23052,22 \\
\hline 070_023 & 22072,11 & 22207,35 & 21697,67 & 21884,67 & 21737,70 & 22291,88 & 21864,34 & 21849,19 \\
\hline 070_024 & 29178,50 & 29145,63 & 28290,05 & 28681,27 & 28689,89 & 28985,19 & 28422,80 & 28422,54 \\
\hline 080_020 & 26866,45 & 26648,19 & 26001,89 & 26262,12 & 26556,38 & 26558,66 & 26450,27 & 26287,59 \\
\hline 080_021 & 18177,00 & 18870,00 & 18088,00 & 17974,00 & 17974,00 & 18290,00 & 18469,00 & 18021,00 \\
\hline 080_022 & 23028,00 & 23020,00 & 22539,42 & 22846,00 & 22614,04 & 22555,00 & 22440,00 & 22555,42 \\
\hline 080_023 & 21593,00 & 21875,88 & 21161,89 & 21608,00 & 21433,65 & 21579,00 & 21581,00 & 21533,44 \\
\hline 080_024 & 27696,37 & 28354,97 & 27281,94 & 27523,24 & 27574,55 & 28111,86 & 28126,29 & 27456,11 \\
\hline
\end{tabular}

Tabela 42: Problemas-teste da classe $(7,5,5)$.

\begin{tabular}{|l|cccccccc|}
\hline & AT1 & AT2 & AT3 & AT4 & AT5 & AT6 & ATH1 & ATH2 \\
\hline 060_025 & 31524,00 & 31110,27 & 30347,88 & 30407,85 & 30932,39 & 30673,00 & 30831,78 & 30376,13 \\
060_026 & 32676,97 & 33395,83 & 31923,64 & 32134,08 & 33426,40 & 32146,51 & 32488,09 & 32083,98 \\
060_027 & 30932,69 & 31968,50 & 30159,45 & 30519,75 & 30707,05 & 31822,00 & 31985,36 & 31985,36 \\
060_028 & 32760,19 & 33714,35 & 31939,57 & 32542,92 & 32681,24 & 33321,21 & 32398,19 & 31947,11 \\
060_029 & - & - & - & - & - & - & - & - \\
070_025 & 30110,86 & 30480,08 & 29463,20 & 29317,49 & 30234,48 & 30109,59 & 30031,84 & 29387,04 \\
070_026 & 32115,00 & 32658,84 & 31415,85 & 32043,60 & 31414,00 & 31597,21 & 31600,91 & 31141,96 \\
070_027 & 29736,27 & 30918,47 & 29219,89 & 29704,38 & 29533,77 & 30252,00 & 29894,51 & 29396,51 \\
070_028 & 32050,00 & 32042,81 & 31146,79 & 31584,00 & 31088,00 & 31528,82 & 31792,49 & 31460,44 \\
070_029 & 32639,01 & 33642,96 & 32402,63 & 32883,89 & 32540,11 & 32500,63 & 32612,23 & 32099,33 \\
080_025 & 29471,00 & 30028,65 & 29166,00 & 29639,00 & 29607,19 & 29762,00 & 29342,00 & 29000,39 \\
080_026 & 31480,00 & 31887,00 & 30932,00 & 31545,00 & 31571,63 & 31232,00 & 31219,99 & 30974,43 \\
080_027 & 29745,00 & 29834,00 & 29059,00 & 30215,00 & 29080,12 & 29163,00 & 28875,00 & 28624,00 \\
080_028 & 31021,04 & 31587,60 & 30688,60 & 31151,13 & 30768,00 & 30898,67 & 31188,76 & 30783,33 \\
080_029 & 32042,82 & 33078,39 & 31618,23 & 31996,23 & 32102,02 & 32574,36 & 31917,05 & 31455,66 \\
\hline
\end{tabular}

Tabela 43: Problemas-teste da classe $(5,7,5)$.

\begin{tabular}{|l|cccccccc|}
\hline & AT1 & AT2 & AT3 & AT4 & AT5 & AT6 & ATH1 & ATH2 \\
\hline 060_030 & - & - & - & - & - & - & - & - \\
060_031 & - & - & - & - & - & - & - & - \\
060_032 & - & - & - & - & - & - & - & - \\
060_033 & - & - & - & - & - & - & - & - \\
060_034 & - & - & - & - & - & - & - & - \\
070_030 & 36799,25 & 37001,00 & 35485,17 & 35943,94 & 35997,41 & 35986,98 & 36190,17 & 35164,07 \\
070_031 & - & - & - & - & - & - & - & - \\
070_032 & 24644,25 & 24912,80 & 24308,83 & 23932,63 & 23751,06 & 24102,74 & 24059,15 & 23807,32 \\
070_033 & - & - & - & - & - & - & - & - \\
070_034 & - & - & - & - & - & - & - & - \\
080_030 & 35220,51 & 35837,86 & 34520,07 & 34992,92 & 34510,66 & 35324,03 & 34948,31 & 34365,52 \\
080_031 & 34646,24 & 35270,00 & 33712,74 & 33958,67 & 34062,69 & 35334,00 & 34405,44 & 33959,00 \\
080_032 & 24349,00 & 24848,20 & 23406,40 & 24184,88 & 24036,94 & 24266,65 & 23979,69 & 23769,64 \\
080_033 & 28908,40 & 30222,04 & 28823,34 & 29332,27 & 28982,94 & 29306,63 & 29500,45 & 28897,87 \\
080_034 & 37648,36 & 38423,29 & 36958,49 & 37118,57 & 37388,98 & 37466,57 & 38108,69 & 37041,46 \\
\hline
\end{tabular}


Tabela 44: Problemas-teste da classe $(5,5,7)$.

\begin{tabular}{|c|cccccccc|}
\hline & AT1 & AT2 & AT3 & AT4 & AT5 & AT6 & ATH1 & ATH2 \\
\hline 060_035 & 34693,90 & 34706,91 & 33592,83 & 34082,32 & 34946,42 & 35265,02 & 33765,04 & 33568,54 \\
060_036 & 33712,33 & 33784,72 & 33304,85 & 33784,85 & 33793,03 & 34292,62 & 33201,35 & 33503,25 \\
060_037 & 33379,28 & 33474,46 & 32303,22 & 32890,14 & 32899,88 & 33359,46 & 32614,32 & 32467,00 \\
060_038 & 33220,93 & 33763,41 & 32330,35 & 32501,72 & 32364,91 & 32888,09 & 32825,67 & 32081,96 \\
060_039 & - & 31836,45 & 30826,00 & 31436,21 & 31362,15 & - & 30827,45 & 30477,42 \\
070_035 & 33823,39 & 33112,65 & 32534,66 & 32793,01 & 32720,11 & 32913,90 & 32880,27 & 32451,14 \\
070_036 & 32888,33 & 33549,49 & 32230,00 & 32956,95 & 33060,93 & 32843,05 & 32877,18 & 32605,27 \\
070_037 & 31131,66 & 32194,74 & 30547,96 & 31662,38 & 31163,13 & 32356,00 & 31364,36 & 31790,96 \\
070_038 & 31985,84 & 32649,29 & 30943,13 & 31633,38 & 30933,13 & 31922,14 & 31612,43 & 31343,71 \\
070_039 & 29844,89 & 30360,54 & 29735,53 & 30318,73 & 29917,86 & 29935,64 & 30053,53 & 29949,86 \\
080_035 & 32722,96 & 32873,15 & 32171,35 & 32627,81 & 32472,33 & 32484,32 & 32321,33 & 32185,15 \\
080_036 & 32173,00 & 32918,42 & 31718,81 & 31964,00 & 32223,54 & 32427,96 & 32154,00 & 31772,90 \\
080_037 & 31069,39 & 31579,85 & 30397,89 & 31211,61 & 30473,82 & 30635,48 & 31092,33 & 30530,48 \\
080_038 & 31216,24 & 32311,73 & 30911,74 & 31142,51 & 31032,39 & 31327,60 & 30975,84 & 30369,16 \\
080_039 & 29496,41 & 30233,78 & 29454,03 & 29750,57 & 29659,44 & 29900,89 & 29395,87 & 29385,70 \\
\hline
\end{tabular}

Tabela 45: Problemas-teste da classe $(7,7,7)$.

\begin{tabular}{|c|cccccccc|}
\hline & AT1 & AT2 & AT3 & AT4 & AT5 & AT6 & ATH1 & ATH2 \\
\hline 060_040 & 64167,00 & 64416,63 & 61385,02 & 62199,65 & 66097,08 & 65235,83 & 62147,01 & 61310,69 \\
060_041 & - & - & - & - & - & - & - & - \\
060_042 & 57900,90 & 58588,95 & 57540,46 & - & 57250,97 & 59698,21 & 56968,97 & 56355,38 \\
060_043 & - & - & - & - & - & - & - & - \\
060_044 & - & - & 65465,17 & 66186,63 & - & - & 65965,53 & 65150,52 \\
070_040 & 62706,31 & 63295,84 & 59492,07 & 60983,00 & 59871,56 & 61925,56 & 61016,94 & 59830,72 \\
070_041 & 65259,90 & 65859,00 & 63011,90 & 64113,76 & 64055,87 & 65582,52 & 64680,76 & 63830,23 \\
070_042 & 57126,28 & 56360,48 & 54517,89 & 55397,66 & 55799,47 & 55975,22 & 55971,89 & 55346,90 \\
070_043 & - & - & - & - & - & - & - & - \\
070_044 & 64476,75 & 65319,58 & 62405,38 & 63235,99 & 63063,67 & 65129,77 & 63728,59 & 62800,34 \\
$080 \_040$ & 59496,55 & 60807,17 & 58204,03 & 60235,03 & 58795,35 & 59980,28 & 59749,08 & 59089,76 \\
$080 \_041$ & 64475,31 & 64471,33 & 61780,02 & 63772,63 & 63011,00 & 63357,00 & 63550,16 & 62164,39 \\
080_042 & 57277,27 & 56845,72 & 54271,50 & 54721,50 & 54902,20 & 56141,17 & 54653,25 & 53777,73 \\
080_043 & 61337,37 & 62857,25 & 59548,66 & 61001,06 & 59894,24 & 61471,84 & 60413,29 & 59483,25 \\
$080 \_044$ & 62775,01 & 64380,65 & 61250,16 & 61782,67 & 61864,12 & 62506,11 & 62261,52 & 61180,52 \\
\hline
\end{tabular}

Tabela 46: Problemas-teste da classe $(10,5,5)$.

\begin{tabular}{|c|c|c|c|c|c|c|c|c|}
\hline & AT1 & AT2 & AT3 & AT4 & AT5 & AT6 & ATH1 & ATH2 \\
\hline $060 \_045$ & 50012,88 & 50614,12 & 49016,63 & 49332,90 & 49526,52 & 50701,17 & 49535,99 & 48943,56 \\
\hline 060_046 & - & 54972,39 & 51964,64 & 52662,18 & - & - & 52109,82 & 52273,06 \\
\hline 060_047 & 50616,00 & 49910,01 & 48244,39 & 48709,01 & 49188,73 & 49448,29 & 49226,63 & 48263,96 \\
\hline 060_048 & 48169,54 & 49048,58 & 47130,18 & 47498,57 & 47901,16 & 49128,11 & 47390,25 & 46966,91 \\
\hline 060_049 & 54085,76 & 54648,92 & 52147,22 & 52822,31 & 53364,54 & 54041,00 & 52526,24 & 52147,15 \\
\hline 070_045 & 48999,90 & 49190,02 & 47224,25 & 47458,20 & 47273,10 & 49573,34 & 47583,67 & 47307,07 \\
\hline 070_046 & 52145,58 & 51868,29 & 50452,47 & 51433,99 & 51534,04 & 52200,39 & 51242,50 & 50895,94 \\
\hline 070_047 & 49190,00 & 49180,38 & 46802,41 & 46757,24 & 47491,50 & 48485,44 & 47530,42 & 46713,20 \\
\hline 070_048 & 47325,50 & 47766,41 & 45889,31 & 46725,26 & 46200,51 & 46931,09 & 4627 & 45602,74 \\
\hline 070_049 & 51878,82 & 52498,10 & 50614,70 & 51217,84 & 51682,63 & 51621,89 & 51565,59 & 51152,67 \\
\hline 080_045 & 47274,00 & 48511,00 & 47000,00 & 47198,00 & 47289,21 & 48341,63 & 47248,00 & 47212,00 \\
\hline 080_046 & 50984,00 & 50839,66 & 50127,28 & 50555,54 & 50586,00 & 50334,92 & 50067,26 & 49454,30 \\
\hline 080_047 & 46817,18 & 47678,00 & 45724,57 & 46332,31 & 46667,00 & 46668 & 458 & 45747,50 \\
\hline 080_048 & 45690,49 & 46904,95 & 45431,78 & 46408,38 & 45584,42 & 46470,97 & 460 & 45789,00 \\
\hline 080_049 & 51084,06 & 51275,90 & 49690,54 & 50157,00 & 50510,97 & 50263,56 & 50457,56 & 49877,29 \\
\hline
\end{tabular}

Tabela 47: Problemas-teste da classe $(5,10,5)$.

\begin{tabular}{|l|cccccccc|}
\hline & AT1 & AT2 & AT3 & AT4 & AT5 & AT6 & ATH1 & ATH2 \\
\hline 060_050 & - & - & - & - & - & - & - & - \\
060_051 & - & - & - & - & - & - & 50362,56 & 49852,53 \\
060_052 & 35613,69 & 36031,95 & 34661,59 & 34235,41 & 35640,92 & 35688,02 & 35596,55 & 35570,41 \\
060_053 & - & - & - & - & - & - & - & - \\
060_054 & - & - & - & - & - & - & - & - \\
070_050 & - & - & - & - & - & - & - & - \\
070_051 & 49221,09 & 50774,86 & 48340,35 & 48321,19 & 48870,88 & 49723,66 & 49922,29 & 48687,39 \\
070_052 & 34078,66 & 35116,11 & 34166,48 & 34166,48 & 34166,48 & 34658,55 & 34584,82 & 33845,39 \\
070_053 & 42965,00 & 42362,50 & 41838,74 & 42348,05 & 41991,06 & 43710,91 & 41389,52 & 41364,59 \\
070_054 & 38172,56 & 40040,44 & 38323,61 & 38322,07 & 38478,39 & 38121,68 & 38637,66 & 38143,66 \\
080_050 & 56299,97 & 56737,71 & 55552,09 & 55324,18 & 56548,70 & 56386,90 & 55599,41 & 55130,93 \\
080_051 & 47323,22 & 49590,75 & 47216,61 & 47601,53 & 47330,81 & 47251,43 & 48104,79 & 47479,69 \\
080_052 & 34114,00 & 35431,78 & 33446,60 & 33714,42 & 34199,96 & 34905,00 & 34678,00 & 33989,91 \\
080_053 & 42270,55 & 42308,24 & 40900,12 & 41733,58 & 41537,92 & 41486,00 & 40887,95 & 40222,58 \\
080_054 & 38151,30 & 39942,00 & 37397,20 & 38020,20 & 38007,58 & 37849,53 & 38103,81 & 38035,81 \\
\hline
\end{tabular}

Tabela 48: Problemas-teste da classe $(5,5,10)$.

\begin{tabular}{|l|cccccccc|}
\hline & AT1 & AT2 & AT3 & AT4 & AT5 & AT6 & ATH1 & ATH2 \\
\hline 060_055 & 48235,81 & 48116,34 & 45437,17 & 46732,18 & 47241,19 & - & 45796,27 & 45098,21 \\
060_056 & - & - & - & - & - & - & 49197,65 & 48840,61 \\
060_057 & - & - & 44430,16 & - & - & - & 44039,36 & 43599,58 \\
060_058 & - & - & - & - & - & - & - & - \\
060_059 & - & - & - & - & - & - & - & - \\
070_055 & 45298,38 & 47075,33 & 44483,72 & 45037,85 & 45001,48 & 46023,45 & 44665,68 & 44591,71 \\
070_056 & 48153,26 & 49082,95 & 47375,31 & 48970,27 & 48916,89 & 49361,66 & 48136,89 & 47750,75 \\
070_057 & 44128,98 & 44000,90 & 42674,94 & 44216,29 & 44220,86 & 44363,32 & 43592,02 & 43153,96 \\
070_058 & 46112,58 & 47051,78 & 45346,55 & 45892,77 & 46175,76 & 47996,37 & 46479,04 & 45696,00 \\
070_059 & 51050,12 & 51467,00 & 49425,60 & 49807,23 & 50090,80 & 50931,43 & 49584,84 & 49699,02 \\
080_055 & 44152,73 & 45119,00 & 43084,66 & 44108,35 & 43783,42 & 43778,98 & 44049,97 & 43614,16 \\
080_056 & 47396,59 & 47877,76 & 46701,40 & 47413,26 & 46993,33 & 47662,06 & 47118,56 & 46195,23 \\
080_057 & 42701,90 & 43996,71 & 41876,92 & 42795,70 & 42753,37 & 42909,33 & 42245,02 & 42560,72 \\
080_058 & 45562,33 & 45973,00 & 44757,60 & 45479,68 & 45076,50 & 44899,90 & 45618,12 & 44967,93 \\
080_059 & 49194,60 & 49877,16 & 48239,17 & 48650,40 & 49390,54 & 49389,21 & 48432,94 & 47775,41 \\
\hline
\end{tabular}


Tabela 49: Problemas-teste da classe $(10,7,7)$.

\begin{tabular}{|l|cccccccc|}
\hline & AT1 & AT2 & AT3 & AT4 & AT5 & AT6 & ATH1 & ATH2 \\
\hline 060_060 & - & - & - & - & - & - & - & - \\
060_061 & 92408,14 & - & 88256,59 & - & 91894,73 & 92704,55 & 91678,06 & 89219,95 \\
060_062 & 90784,41 & 91728,72 & 85280,22 & 86594,32 & 90099,94 & 91184,40 & 88175,46 & 84932,11 \\
060_063 & - & - & - & - & - & - & - & - \\
060_064 & 101342,76 & 103207,76 & 98025,08 & 99833,29 & 102919,51 & 101918,92 & 99837,98 & 96910,09 \\
070_060 & 99095,00 & 98868,83 & 93100,11 & 93871,67 & 94906,32 & 98646,06 & 95057,78 & 94439,47 \\
070_061 & 89123,03 & 90241,17 & 84619,07 & 85883,23 & 88157,00 & 88671,00 & 85935,84 & 84905,06 \\
070_062 & 84272,36 & 87029,22 & 82697,69 & 83571,00 & 83864,37 & 84461,07 & 85495,86 & 83232,87 \\
070_063 & 95483,64 & 94258,66 & 91526,87 & 92629,93 & 92040,65 & 95219,20 & 93086,34 & 91547,84 \\
070_064 & 99420,33 & 99661,60 & 93919,43 & 94334,27 & 96481,79 & 98017,15 & 96606,24 & 94737,91 \\
080_060 & 93514,70 & 94993,12 & 91492,47 & 93065,74 & 92787,12 & 93769,88 & 93622,66 & 92709,24 \\
080_061 & 84621,60 & 88593,63 & 82465,18 & 84537,75 & 84623,57 & 85521,47 & 85084,59 & 83300,48 \\
080_062 & 82628,19 & 86153,28 & 81947,77 & 83900,68 & 83870,10 & 82920,91 & 84869,21 & 82302,56 \\
080_063 & 93322,45 & 92179,59 & 87846,50 & 90545,46 & 89901,78 & 90525,91 & 90056,54 & 89242,67 \\
080_064 & 94569,30 & 97132,51 & 93060,13 & 94560,68 & 93510,33 & 96432,23 & 95336,78 & 93803,74 \\
\hline
\end{tabular}

Tabela 50: Problemas-teste da classe $(7,10,7)$.

\begin{tabular}{|c|c|c|c|c|c|c|c|c|}
\hline & AT1 & AT2 & AT3 & AT4 & AT5 & AT6 & ATH1 & ATH2 \\
\hline 060_065 & - & - & 92222,97 & - & - & - & 90360,74 & 89842,79 \\
\hline 060_066 & - & - & - & - & - & - & - & - \\
\hline 060_067 & - & - & - & - & - & - & - & - \\
\hline 060_068 & - & - & - & - & - & - & - & - \\
\hline 060_069 & - & - & - & - & - & - & - & - \\
\hline 070_065 & 91522,95 & 91464,41 & 88888,87 & 89650,30 & 89819,52 & 90617,02 & 89028,85 & 89197,36 \\
\hline 070_066 & 95045,15 & 95230,31 & 91219,96 & 91949,81 & 93050,40 & 94778,54 & 92412,45 & 91084,71 \\
\hline 070_067 & 83983,54 & 85178,43 & 78592,46 & 80767,86 & 81684,02 & 82933,53 & 79384,60 & 77975,09 \\
\hline 070_068 & 95316,00 & 94996,65 & 90948,16 & 92477,36 & 92641,61 & 95121,55 & 92638,81 & 91777,01 \\
\hline 070_069 & - & - & - & - & - & - & - & - \\
\hline 080_065 & 88808,19 & 90419,02 & 87557,58 & 88940,42 & 87655,01 & 89765,15 & 87970,81 & 87322,28 \\
\hline 080_066 & 91918,05 & 93117,06 & 88749,74 & 88871,33 & 91178,19 & 91385,00 & 91497,31 & 89752,48 \\
\hline 080_067 & 79485,50 & 80748,10 & 76346,58 & 77033,45 & 78054,68 & 78766,13 & 78170,31 & 76834,18 \\
\hline 080_068 & 92241,52 & 93837,86 & 88299,67 & 90936,88 & 89227,94 & 90560,42 & 91421,34 & 88469,09 \\
\hline 080_069 & 101654,25 & 102838,96 & 98727,09 & 100015,67 & 100357,25 & 101412,30 & 98366,06 & 97976,09 \\
\hline
\end{tabular}

Tabela 51: Problemas-teste da classe $(7,7,10)$.

\begin{tabular}{|c|c|c|c|c|c|c|c|c|}
\hline & AT1 & AT2 & AT3 & AT4 & AT5 & AT6 & ATH1 & ATH2 \\
\hline 060_070 & - & - & - & - & - & - & - & - \\
\hline 060_071 & 84814,28 & 86590,44 & 81841,96 & 83599,99 & 86189,36 & 86246,43 & 84593,74 & 84262,17 \\
\hline 060_072 & - & - & 96939,35 & - & - & - & 96915,11 & 96029,17 \\
\hline 060_073 & - & - & - & - & - & - & - & 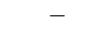 \\
\hline 060_074 & - & - & - & - & - & - & 98380,84 & 96692,21 \\
\hline 070_070 & 97775,70 & 97750,43 & 94301,20 & 94940,26 & 96915,70 & 97949,16 & 96105,89 & 94525,54 \\
\hline 070_071 & 83138,55 & 83642,84 & 80084,78 & 80447,64 & 79912,72 & 82803,00 & 82351,98 & 80077,29 \\
\hline 070_072 & 96103,99 & 97349,32 & 91926,24 & 94019,11 & 94398,15 & 97877,42 & 94704,41 & 91994,81 \\
\hline 070_073 & 98224,00 & 98611,33 & 94165,44 & 95985,69 & 96125,00 & 97721,86 & 96335,95 & 94021,86 \\
\hline 070_074 & 98830,26 & 97655,79 & 93880,23 & 93905,21 & 96198,56 & 98842,90 & 96034,06 & 93605,05 \\
\hline 080_070 & 94876,82 & 95129,56 & 92437,46 & 94183,16 & 92275,72 & 93884,87 & 94843,70 & 93127,71 \\
\hline 080_071 & 80094,51 & 82634,00 & 78768,65 & 79271,42 & 79313,73 & 79049,92 & 80613,31 & 78881,98 \\
\hline 080_072 & 93063,48 & 94730,80 & 90415,12 & 92190,00 & 91278,49 & 92449,85 & 92750,33 & 90598,00 \\
\hline 080_073 & 94319,59 & 95884,22 & 92205,24 & 93835,09 & 93819,18 & 93845,44 & 93274,73 & 92323,68 \\
\hline 080_074 & 93538,20 & 94669,02 & 91458,52 & 91964,92 & 93817,20 & 93413,29 & 93060,69 & 91762,19 \\
\hline
\end{tabular}

Tabela 52: Problemas-teste da classe $(10,10,10)$.

\begin{tabular}{|l|cccccccc|}
\hline & AT1 & AT2 & AT3 & AT4 & AT5 & AT6 & ATH1 & ATH2 \\
\hline 060_075 & - & - & - & - & - & - & 182179,97 & 178128,20 \\
060_076 & - & - & - & - & - & - & 191228,94 & 190292,86 \\
060_077 & - & - & - & - & - & - & - & - \\
060_078 & - & - & - & - & - & - & - & - \\
060_079 & - & - & - & - & - & - & - \\
070_075 & 180594,62 & 179929,74 & 172100,72 & 176118,49 & 179995,45 & 180401,68 & 178245,64 & 174003,33 \\
070_076 & 186535,30 & 191606,17 & 179431,52 & 180928,14 & 188891,46 & 190538,42 & 189127,14 & 183894,26 \\
070_077 & 206546,05 & 208205,64 & 196251,53 & 200651,71 & 206435,12 & 209451,20 & 203368,89 & 200246,91 \\
070_078 & 216377,05 & 219608,59 & 209490,33 & 210559,93 & 216925,94 & 218946,22 & 213771,75 & 211378,38 \\
070_079 & 208021,53 & 208258,15 & 197998,56 & 201732,67 & 205685,06 & 211180,67 & 201540,03 & 200210,85 \\
080_075 & 176182,46 & 178088,39 & 169215,75 & 173220,05 & 173466,62 & 173237,84 & 174760,34 & 171059,55 \\
080_076 & 182541,06 & 189421,36 & 175172,07 & 179408,51 & 179342,87 & 178302,16 & 185844,77 & 176988,71 \\
080_077 & 199777,04 & 202632,23 & 192419,91 & 195361,09 & 194065,21 & 198286,51 & 200054,23 & 194607,38 \\
080_078 & 210164,31 & 214854,52 & 202751,81 & 204820,24 & 205825,11 & 212832,04 & 209225,53 & 206824,27 \\
080_079 & 201159,10 & 204334,24 & 192907,21 & 195180,30 & 199592,12 & 202720,72 & 200715,97 & 193759,70 \\
\hline
\end{tabular}

Tabela 53: Problemas-teste da classe $(15,10,10)$.

\begin{tabular}{|c|cccccccc|}
\hline & AT1 & AT2 & AT3 & AT4 & AT5 & AT6 & ATH1 & ATH2 \\
\hline 060_080 & - & - & - & - & - & - & 307991,47 & 305883,94 \\
060_081 & - & - & 286586,29 & - & - & - & 286675,09 & 281968,44 \\
060_082 & - & - & 308353,62 & 312675,94 & - & - & 310679,91 & 307196,27 \\
060_083 & 265487,17 & 267056,60 & 251169,54 & 253281,72 & 259424,43 & 268556,48 & 256991,22 & 253919,15 \\
060_084 & - & - & 297592,96 & 302779,83 & - & - & 298063,88 & 296898,51 \\
070_080 & 310764,63 & 311403,73 & 291404,36 & 296121,72 & 306242,46 & 310748,10 & 303059,63 & 300954,02 \\
070_081 & 288289,50 & 289809,67 & 267631,16 & 273422,17 & 277624,04 & 288855,85 & 283262,63 & 277963,71 \\
070_082 & 308857,75 & 310951,14 & 293849,62 & 295985,25 & 302223,45 & 310047,64 & 304693,38 & 303329,98 \\
070_083 & 257800,00 & 260369,25 & 241166,69 & 245636,34 & 248537,85 & 250841,84 & 252858,17 & 246301,28 \\
070_084 & 295664,66 & 295714,26 & 281228,56 & 282520,86 & 284296,06 & 297153,02 & 294574,25 & 290541,25 \\
080_080 & 302178,29 & 306504,70 & 284852,91 & 290470,66 & 291194,26 & 301103,08 & 300034,47 & 290690,17 \\
080_081 & 282984,42 & 285106,45 & 264550,09 & 272287,87 & 276931,30 & 274097,65 & 279400,28 & 274450,20 \\
080_082 & 305200,24 & 309265,96 & 286408,91 & 290312,94 & 301635,06 & 298632,85 & 300494,06 & 293801,35 \\
080_083 & 246803,83 & 256105,53 & 238739,95 & 243230,98 & 248806,08 & 247972,14 & 250735,17 & 239142,86 \\
080_084 & 290565,76 & 287904,62 & 275266,76 & 279626,44 & 283558,42 & 289871,52 & 288827,97 & 279229,90 \\
\hline
\end{tabular}


Tabela 54: Problemas-teste da classe $(10,15,10)$.

\begin{tabular}{|c|c|c|c|c|c|c|c|c|}
\hline & AT1 & AT2 & AT3 & AT4 & AT5 & AT6 & ATH1 & ATH2 \\
\hline 060_085 & - & - & - & - & - & - & - & - \\
\hline 060_086 & - & - & - & - & - & - & - & - \\
\hline 060_087 & - & - & - & - & - & - & - & - \\
\hline 060_088 & - & - & - & - & - & - & - & - \\
\hline 060_089 & - & - & - & - & - & - & - & - \\
\hline 070_085 & 295729,32 & 295907,93 & 281110,32 & 283924,11 & 291179,85 & - & 288118,22 & 286274,11 \\
\hline 070_086 & 294947,07 & 294804,71 & 281989,51 & 286120,48 & 293154,79 & 298963,93 & 289288,09 & 283531,71 \\
\hline 070_087 & 325323,13 & - & 316663,96 & 318319,56 & - & - & 317763,75 & 316123,79 \\
\hline 070_088 & - & - & - & - & - & - & - & - \\
\hline 070_089 & - & - & 305522,07 & 309899,15 & - & - & 308027,56 & 306906,86 \\
\hline 080_085 & 289485,92 & 285387,12 & 271492,86 & 274983,81 & 278150,72 & 282577,35 & 284077,16 & 275556,80 \\
\hline 080_086 & 289898,81 & 291703,61 & 275279,42 & 280637,75 & 282244,66 & 283201,47 & 283016,81 & 279728,62 \\
\hline 080_087 & 320715,65 & 320996,06 & 300647,87 & 307635,33 & 310919,91 & 313230,68 & 316384,22 & 311149,68 \\
\hline 080_088 & 301048,05 & 303540,69 & 287819,76 & 293283,00 & 296434,97 & 301448,96 & 292681,03 & 289860,10 \\
\hline 080_089 & 310688,65 & 312903,53 & 292753,29 & 298867,80 & 305220,64 & 309944,65 & 305171,84 & 301561,01 \\
\hline
\end{tabular}

Tabela 55: Problemas-teste da classe $(10,10,15)$.

\begin{tabular}{|c|c|c|c|c|c|c|c|c|}
\hline & AT1 & AT2 & AT3 & AT4 & AT5 & AT6 & ATH1 & ATH2 \\
\hline$\overline{060 \_090}$ & - & - & - & - & - & - & - & - \\
\hline 060_091 & - & - & - & - & - & - & 283205,78 & 282199,17 \\
\hline 060_092 & - & - & - & - & - & - & - & - \\
\hline 060_093 & - & - & - & - & - & - & - & - \\
\hline 060_094 & - & - & - & - & - & - & 300772,63 & 299368,72 \\
\hline 070_090 & 317965,83 & 317186,03 & 304063,40 & 308257,58 & 311961,08 & 320837,41 & 310396,88 & 308825,91 \\
\hline 070_091 & 284500,77 & 286381,50 & 270742,16 & 274664,49 & 282228,63 & 285880,45 & 280033,44 & 273366,13 \\
\hline 070_092 & 322115,60 & 321323,16 & 307793,67 & 310054,73 & 319772,74 & 321754,16 & 313312,34 & 311167,22 \\
\hline 070_093 & 277188,12 & 283400,58 & 265864,83 & 267871,28 & 277466,08 & 284329,73 & 276503,69 & 270461,35 \\
\hline 070_094 & 301769,06 & 300567,37 & 283617,68 & 286382,74 & 293965,80 & 301180,03 & 296969,78 & 292563,50 \\
\hline 080_090 & 311928,44 & 312055,42 & 297027,73 & 303976,63 & 308676,67 & 307312,05 & 307652,16 & 303694,80 \\
\hline 080_091 & 279450,25 & 282239,48 & 262660,11 & 268910,78 & 277582,25 & 280480,11 & 275037,69 & 265458,78 \\
\hline 080_092 & 315547,16 & 315110,78 & 299175,72 & 303083,84 & 310236,91 & 312885,54 & 308811,75 & 306477,53 \\
\hline 080_093 & 270562,85 & 274570,84 & 259298,69 & 262877,84 & 263278,37 & 267341,18 & 272559,84 & 262153,70 \\
\hline 080_094 & 295557,75 & 297058,10 & 277184,50 & 282936,79 & 286358,12 & 288278,12 & 292785,66 & 290954,05 \\
\hline
\end{tabular}

Tabela 56: Problemas-teste da classe $(15,15,15)$.

\begin{tabular}{|c|c|c|c|c|c|c|c|c|}
\hline & AT1 & AT2 & AT3 & AT4 & AT5 & AT6 & ATH1 & ATH2 \\
\hline 060_095 & - & - & - & - & - & - & - & - \\
\hline 060_096 & - & - & - & - & - & - & - & - \\
\hline 060_097 & - & - & - & - & - & - & - & 683110,18 \\
\hline 060_098 & - & - & - & - & - & - & - & - \\
\hline 060_099 & - & - & - & - & - & - & 692243,88 & 684813,33 \\
\hline 070_095 & 661279,29 & 660556,61 & 621030,30 & 628129,61 & 652229,08 & 657786,04 & 649802,56 & 640407,83 \\
\hline 070_096 & 678175,42 & 680383,68 & 645696,66 & 652011,61 & 675924,09 & 677016,50 & 665094,94 & 653477,80 \\
\hline 070_097 & 685645,44 & 690837,81 & 653841,70 & 656114,20 & 681973,74 & 689015,04 & 679452,56 & 671119,20 \\
\hline 070_098 & 656852,79 & 660459,46 & 616674,15 & 622644,94 & 641246,09 & 654995,84 & 642814,25 & 637367,14 \\
\hline 070_099 & 692125,60 & 694422,50 & 656756,75 & 661828,50 & 689689,85 & 692696,76 & 682974,56 & 674900,81 \\
\hline 080_095 & 642555,69 & 655327,76 & 608232,42 & 612107,45 & 642306,32 & 638814,48 & 644892,50 & 629835,55 \\
\hline 080_096 & 664823,60 & 665453,81 & 628564,21 & 635308,20 & 656675,74 & 660334,48 & 657977,88 & 645398,57 \\
\hline 080_097 & 670280,29 & 678047,38 & 635320,30 & 645675,09 & 651413,89 & 662295,57 & 668725,13 & 657187,61 \\
\hline 080_098 & 651920,64 & 649890,17 & 600816,11 & 614459,59 & 628663,53 & 641512,95 & 637594,00 & 624139,51 \\
\hline 080_099 & 679122,38 & 679498,81 & 641768,53 & 647587,72 & 670626,40 & 678887,39 & 673426,81 & 667941,33 \\
\hline
\end{tabular}




\section{APÊNDICE D - Tempos Computacionais}

As tabelas de tempos computacionais das heurísticas da literatura e das metaheurísticas propostas, analogamente aos resultados computacionais, foram divididos por classe de problemas-teste. Em todas tabelas, a primeira coluna define o código do problema-teste e as colunas seguintes apresentam os tempos computacionais das abordagens propostas e da literatura. Nesse caso, todo teste tem seu tempo computacional apresentado, mesmo que nenhuma solução tenha sido encontrada.

Tabela 57: Problemas-teste da classe $(3,3,3)$.

\begin{tabular}{c|ccccccc}
\hline Tempo & MIP & LB1 & LB2 & RH1 & RH2 & RH3 & RF1 \\
\hline 060_000 & 0,87 & 0,02 & 0,19 & 0,37 & 0,69 & 0,14 & 0,58 \\
060_001 & 0,66 & 0,05 & 0,16 & 0,34 & 0,78 & 0,05 & 0,48 \\
060_002 & 0,45 & 0,06 & 0,22 & 0,45 & 0,53 & 0,06 & 0,66 \\
060_003 & 0,53 & 0,03 & 0,27 & 0,61 & 0,69 & 0,12 & 0,69 \\
060_004 & 0,72 & 0,06 & 0,16 & 0,61 & 0,44 & 0,12 & 0,72 \\
070_000 & 0,34 & 0,05 & 0,14 & 0,31 & 0,47 & 0,11 & 0,67 \\
070_001 & 0,36 & 0,03 & 0,14 & 0,41 & 0,39 & 0,08 & 0,67 \\
070_002 & 0,34 & 0,05 & 0,14 & 0,39 & 0,47 & 0,08 & 0,69 \\
070_003 & 0,30 & 0,02 & 0,16 & 0,30 & 0,30 & 0,06 & 0,76 \\
070_004 & 0,30 & 0,05 & 0,14 & 0,30 & 0,37 & 0,09 & 0,69 \\
080_000 & 0,39 & 0,03 & 0,14 & 0,64 & 0,55 & 0,09 & 0,50 \\
080_001 & 0,28 & 0,00 & 0,14 & 0,37 & 0,44 & 0,08 & 0,45 \\
080_002 & 0,30 & 0,05 & 0,09 & 0,47 & 0,66 & 0,05 & 0,44 \\
080_003 & 0,30 & 0,03 & 0,12 & 0,61 & 0,41 & 0,09 & 0,50 \\
080_004 & 0,28 & 0,02 & 0,12 & 0,39 & 0,37 & 0,05 & 0,39 \\
\hline
\end{tabular}

Tabela 58: Problemas-teste da classe $(5,3,3)$.

\begin{tabular}{c|ccccccc}
\hline Tempo & MIP & LB1 & LB2 & RH1 & RH2 & RH3 & RF1 \\
\hline 060_005 & 6,71 & 0,09 & 0,80 & 3,53 & 3,51 & 0,09 & 0,76 \\
060_006 & 12,71 & 0,11 & 0,72 & 1,73 & 1,64 & 0,11 & 2,11 \\
060_007 & 36,60 & 0,08 & 0,61 & 2,61 & 2,76 & 0,12 & 2,48 \\
060_008 & 37,66 & 0,09 & 0,47 & 2,32 & 2,67 & 0,16 & 2,14 \\
060_009 & 49,20 & 0,06 & 0,84 & 2,15 & 2,18 & 0,16 & 1,81 \\
070_005 & 14,13 & 0,06 & 1,00 & 1,76 & 2,85 & 0,16 & 1,87 \\
070_006 & 4,40 & 0,05 & 0,70 & 1,61 & 1,64 & 0,12 & 1,39 \\
070_007 & 17,99 & 0,06 & 0,50 & 2,09 & 2,64 & 0,16 & 1,79 \\
070_008 & 20,42 & 0,08 & 0,41 & 2,03 & 2,96 & 0,12 & 1,81 \\
070_009 & 3,92 & 0,06 & 0,67 & 1,36 & 1,79 & 0,16 & 1,95 \\
080_005 & 12,42 & 0,06 & 0,69 & 1,83 & 1,83 & 0,16 & 1,58 \\
080_006 & 1,79 & 0,05 & 0,51 & 1,87 & 1,31 & 0,12 & 1,54 \\
080_007 & 9,08 & 0,05 & 0,51 & 1,81 & 2,12 & 0,17 & 1,79 \\
080_008 & 4,20 & 0,06 & 0,48 & 2,00 & 1,76 & 0,14 & 1,31 \\
080_009 & 14,46 & 0,05 & 0,56 & 1,97 & 2,18 & 0,14 & 1,45 \\
\hline
\end{tabular}


Tabela 59: Problemas-teste da classe $(3,5,3)$.

\begin{tabular}{|c|c|c|c|c|c|c|c|}
\hline Tempo & MIP & LB1 & LB2 & RH1 & RH2 & RH3 & RF1 \\
\hline 060_010 & 0,70 & 0,05 & 0,39 & 0,66 & 0,64 & 0,17 & 0,87 \\
\hline 060_011 & 0,11 & 0,05 & 0,09 & 0,05 & 0,09 & 0,03 & 0,08 \\
\hline 060_012 & 0,90 & 0,09 & 0,37 & 0,97 & 0,80 & 0,08 & 0,80 \\
\hline 060_013 & 0,16 & 0,08 & 0,25 & 0,11 & 0,14 & 0,06 & 0,23 \\
\hline 060_014 & 0,64 & 0,08 & 0,30 & 0,84 & 0,69 & 0,12 & 0,72 \\
\hline 070_010 & 0,48 & 0,03 & 0,30 & 0,36 & 0,67 & 0,06 & 1,26 \\
\hline 070_011 & 0,05 & 0,05 & 0,20 & 0,06 & 0,06 & 0,06 & 0,23 \\
\hline 070_012 & 0,48 & 0,02 & 0,23 & 0,67 & 0,56 & 0,11 & 0,67 \\
\hline 070_013 & 0,16 & 0,03 & 0,23 & 0,19 & 0,17 & 0,08 & 0,31 \\
\hline 070_014 & 0,34 & 0,03 & 0,25 & 0,55 & 0,55 & 0,11 & 0,61 \\
\hline 080_010 & 0,44 & 0,02 & 0,20 & 0,64 & 0,50 & 0,11 & 0,78 \\
\hline 080_011 & 0,42 & 0,05 & 0,31 & 0,55 & 0,61 & 0,09 & 0,59 \\
\hline 080_012 & 0,45 & 0,05 & 0,19 & 0,75 & 0,56 & 0,11 & 0,64 \\
\hline 080_013 & 0,33 & 0,03 & 0,20 & 0,69 & 0,76 & 0,09 & 0,48 \\
\hline 080_014 & 0,41 & 0,02 & 0,20 & 0,69 & 0,78 & 0,08 & 0,53 \\
\hline
\end{tabular}

Tabela 60: Problemas-teste da classe $(3,3,5)$.

\begin{tabular}{c|ccccccc}
\hline Tempo & MIP & LB1 & LB2 & RH1 & RH2 & RH3 & RF1 \\
\hline 060_015 & 1,39 & 0,08 & 0,41 & 0,67 & 1,22 & 0,14 & 1,47 \\
060_016 & 0,48 & 0,05 & 0,51 & 0,19 & 0,22 & 0,06 & 0,14 \\
060_017 & 0,17 & 0,06 & 0,66 & 0,14 & 0,12 & 0,08 & 0,33 \\
060_018 & 0,73 & 0,06 & 0,30 & 0,64 & 0,81 & 0,17 & 1,84 \\
060_019 & 0,16 & 0,06 & 0,36 & 0,12 & 0,11 & 0,08 & 0,33 \\
$070 \_015$ & 0,87 & 0,03 & 0,37 & 0,75 & 0,97 & 0,14 & 1,39 \\
070_016 & 0,78 & 0,05 & 0,33 & 0,51 & 0,66 & 0,17 & 1,15 \\
070_017 & 1,44 & 0,03 & 0,55 & 0,86 & 1,01 & 0,16 & 1,54 \\
$070 \_018$ & 0,72 & 0,02 & 0,39 & 0,47 & 0,78 & 0,16 & 1,54 \\
$070 \_019$ & 1,15 & 0,05 & 0,37 & 0,59 & 0,78 & 0,16 & 1,67 \\
$080 \_015$ & 0,76 & 0,02 & 0,31 & 1,12 & 1,08 & 0,14 & 0,98 \\
080_016 & 0,56 & 0,05 & 0,31 & 0,80 & 0,80 & 0,14 & 0,84 \\
$080 \_017$ & 0,80 & 0,05 & 0,47 & 0,69 & 0,76 & 0,16 & 0,86 \\
$080 \_018$ & 0,59 & 0,03 & 0,28 & 0,78 & 0,73 & 0,11 & 0,80 \\
$080 \_019$ & 0,97 & 0,02 & 0,31 & 0,61 & 0,90 & 0,14 & 0,92 \\
\hline
\end{tabular}

Tabela 61: Problemas-teste da classe $(5,5,5)$.

\begin{tabular}{c|ccccccc}
\hline Tempo & MIP & LB1 & LB2 & RH1 & RH2 & RH3 & RF1 \\
\hline 060_020 & 7200,04 & 0,31 & 13,45 & 45,71 & 42,00 & 0,98 & 2,95 \\
060_021 & 7200,05 & 0,23 & 64,10 & 20,30 & 17,53 & 0,42 & 6,85 \\
060_022 & 3303,01 & 0,31 & 17,22 & 45,12 & 43,98 & 0,28 & 2,20 \\
060_023 & 7200,05 & 0,20 & 16,26 & 28,38 & 26,07 & 0,30 & 2,29 \\
060_024 & 35,55 & 0,30 & 10,09 & 36,07 & 36,15 & 0,36 & 3,63 \\
070_020 & 7200,02 & 0,23 & 23,04 & 17,28 & 22,54 & 0,58 & 6,90 \\
070_021 & 7200,02 & 0,19 & 28,91 & 11,29 & 11,15 & 0,56 & 4,91 \\
070_022 & 1611,55 & 0,27 & 32,34 & 19,34 & 18,66 & 0,58 & 5,82 \\
070_023 & 5981,59 & 0,27 & 26,40 & 11,15 & 12,59 & 0,50 & 6,19 \\
070_024 & 7200,12 & 0,27 & 27,85 & 62,62 & 55,69 & 0,72 & 7,92 \\
080_020 & 3704,62 & 0,23 & 22,99 & 13,65 & 15,02 & 0,55 & 6,88 \\
080_021 & 769,66 & 0,14 & 17,14 & 6,82 & 6,51 & 0,41 & 5,23 \\
080_022 & 2161,41 & 0,23 & 31,51 & 9,34 & 9,19 & 0,39 & 6,02 \\
080_023 & 117,70 & 0,19 & 28,00 & 12,60 & 13,67 & 0,44 & 5,77 \\
080_024 & 7200,01 & 0,27 & 25,01 & 27,46 & 38,36 & 0,56 & 6,04 \\
\hline
\end{tabular}

Tabela 62: Problemas-teste da classe $(7,5,5)$.

\begin{tabular}{c|ccccccc}
\hline Tempo & MIP & LB1 & LB2 & RH1 & RH2 & RH3 & RF1 \\
\hline 060_025 & 7200,10 & 0,73 & 321,95 & 302,58 & 329,22 & 1,22 & 23,03 \\
060_026 & 7200,04 & 0,86 & 444,52 & 393,62 & 354,65 & 1,22 & 27,13 \\
060_027 & 7200,09 & 0,86 & 223,38 & 249,12 & 294,36 & 1,59 & 23,38 \\
060_028 & 7200,05 & 1,00 & 365,84 & 448,78 & 476,08 & 1,50 & 31,70 \\
060_029 & 7200,10 & 1,05 & 349,65 & 1196,51 & 908,04 & 0,95 & 43,71 \\
070_025 & 7200,09 & 0,55 & 207,42 & 223,69 & 228,73 & 1,12 & 21,11 \\
070_026 & 7200,05 & 0,80 & 354,40 & 247,14 & 307,60 & 1,06 & 25,66 \\
070_027 & 7200,30 & 0,66 & 194,27 & 438,35 & 483,63 & 1,15 & 20,36 \\
070_028 & 7200,09 & 0,61 & 367,44 & 348,79 & 330,58 & 1,23 & 25,83 \\
070_029 & 7200,04 & 0,72 & 265,81 & 373,90 & 575,49 & 1,25 & 29,61 \\
080_025 & 7200,04 & 0,58 & 211,12 & 219,20 & 241,82 & 1,01 & 18,63 \\
080_026 & 7200,10 & 0,61 & 253,84 & 293,06 & 257,60 & 1,01 & 23,88 \\
080_027 & 7200,02 & 0,61 & 160,95 & 167,12 & 184,08 & 1,14 & 19,69 \\
080_028 & 7200,07 & 0,67 & 230,93 & 230,10 & 183,52 & 1,11 & 24,52 \\
080_029 & 7200,07 & 0,61 & 161,71 & 229,43 & 186,44 & 1,11 & 25,65 \\
\hline
\end{tabular}

Tabela 63: Problemas-teste da classe $(5,7,5)$.

\begin{tabular}{c|ccccccc}
\hline Tempo & MIP & LB1 & LB2 & RH1 & RH2 & RH3 & RF1 \\
\hline 060_030 & 125,83 & 0,58 & 30,73 & 77,70 & 77,59 & 0,97 & 3,96 \\
060_031 & 105,83 & 0,59 & 30,17 & 121,70 & 121,40 & 0,66 & 5,02 \\
060_032 & 109,09 & 0,41 & 19,92 & 54,93 & 54,96 & 0,51 & 4,07 \\
060_033 & 30,11 & 0,47 & 16,74 & 19,69 & 19,58 & 0,48 & 4,24 \\
060_034 & 77,69 & 0,62 & 21,53 & 8,60 & 8,47 & 0,19 & 3,24 \\
070_030 & 7200,09 & 0,56 & 16,68 & 61,28 & 70,22 & 0,92 & 12,40 \\
070_031 & 205,30 & 0,45 & 51,65 & 75,02 & 74,94 & 0,50 & 3,79 \\
070_032 & 1868,95 & 0,37 & 32,89 & 27,35 & 26,94 & 0,70 & 8,83 \\
070_033 & 130,73 & 0,42 & 57,13 & 63,18 & 63,23 & 0,50 & 4,49 \\
070_034 & 173,04 & 0,45 & 60,59 & 79,75 & 79,59 & 0,56 & 2,68 \\
080_030 & 7200,02 & 0,34 & 28,28 & 64,21 & 68,58 & 0,69 & 10,44 \\
080_031 & 7200,07 & 0,34 & 34,80 & 43,06 & 51,37 & 0,76 & 8,81 \\
080_032 & 2537,11 & 0,34 & 70,89 & 37,38 & 38,33 & 0,51 & 7,60 \\
080_033 & 7200,04 & 0,36 & 99,08 & 23,01 & 20,37 & 0,66 & 9,98 \\
080_034 & 7200,02 & 0,53 & 64,38 & 59,26 & 52,76 & 0,78 & 10,47 \\
\hline
\end{tabular}


Tabela 64: Problemas-teste da classe $(5,5,7)$.

\begin{tabular}{c|ccccccc}
\hline Tempo & MIP & LB1 & LB2 & RH1 & RH2 & RH3 & RF1 \\
\hline 060_035 & 7200,07 & 0,55 & 162,07 & 43,87 & 64,46 & 1,22 & 15,51 \\
060_036 & 7200,05 & 0,55 & 783,03 & 60,65 & 83,63 & 1,29 & 16,30 \\
060_037 & 7200,02 & 0,47 & 227,04 & 43,73 & 44,41 & 0,45 & 5,34 \\
060_038 & 7200,09 & 0,48 & 341,36 & 42,45 & 45,71 & 1,31 & 15,30 \\
060_039 & 7200,07 & 0,50 & 375,10 & 77,36 & 52,43 & 1,12 & 16,10 \\
070_035 & 7200,09 & 0,37 & 104,43 & 40,67 & 30,11 & 0,95 & 12,82 \\
070_036 & 7200,05 & 0,39 & 324,17 & 45,01 & 58,28 & 1,15 & 14,04 \\
070_037 & 7200,02 & 0,36 & 169,93 & 38,41 & 33,59 & 1,06 & 13,35 \\
070_038 & 7200,02 & 0,37 & 400,69 & 43,70 & 29,31 & 1,03 & 11,65 \\
070_039 & 7200,05 & 0,33 & 203,69 & 24,68 & 31,98 & 0,98 & 12,09 \\
080_035 & 7200,04 & 0,33 & 80,57 & 29,19 & 27,74 & 0,90 & 12,48 \\
080_036 & 7200,13 & 0,41 & 172,99 & 24,68 & 22,50 & 0,95 & 11,76 \\
080_037 & 7200,01 & 0,39 & 114,04 & 31,61 & 23,10 & 0,90 & 11,11 \\
080_038 & 7200,07 & 0,27 & 192,88 & 34,29 & 28,36 & 0,78 & 10,83 \\
$080 \_039$ & 7200,07 & 0,33 & 112,48 & 24,79 & 21,36 & 0,94 & 11,22 \\
\hline
\end{tabular}

Tabela 65: Problemas-teste da classe $(7,7,7)$.

\begin{tabular}{c|ccccccc}
\hline Tempo & MIP & LB1 & LB2 & RH1 & RH2 & RH3 & RF1 \\
\hline 060_040 & 7200,05 & 3,31 & 7200,12 & 2956,42 & 3129,24 & 7,49 & 181,37 \\
060_041 & 7200,04 & 3,96 & 7200,09 & 1028,84 & 1029,11 & 6,57 & 54,40 \\
060_042 & 7200,04 & 3,40 & 7200,02 & 2515,30 & 2445,21 & 4,96 & 121,85 \\
060_043 & 461,01 & 3,17 & 2283,76 & 172,04 & 172,15 & 1,37 & 33,49 \\
060_044 & 7200,12 & 3,42 & 7200,04 & 2057,20 & 3614,78 & 8,66 & 188,34 \\
070_040 & 7200,13 & 2,51 & 7200,38 & 2014,28 & 1532,83 & 5,46 & 131,48 \\
070_041 & 7200,07 & 2,54 & 7200,09 & 1611,69 & 3154,73 & 4,35 & 122,43 \\
070_042 & 7200,07 & 2,34 & 7200,15 & 1899,45 & 2220,42 & 3,92 & 76,61 \\
070_043 & 7200,15 & 1,97 & 7200,04 & 1028,64 & 1028,61 & 3,63 & 29,53 \\
070_044 & 7200,13 & 2,48 & 7200,09 & 2692,94 & 2419,28 & 5,69 & 127,92 \\
080_040 & 7200,09 & 2,26 & 7200,04 & 1386,44 & 1120,88 & 4,38 & 104,30 \\
080_041 & 7200,16 & 2,59 & 7200,05 & 1351,30 & 1316,84 & 3,56 & 92,91 \\
080_042 & 7200,10 & 3,06 & 7200,05 & 1314,53 & 1508,31 & 3,85 & 71,84 \\
080_043 & 7200,21 & 1,90 & 7148,96 & 1212,39 & 1023,69 & 3,49 & 77,30 \\
080_044 & 7200,13 & 2,67 & 4673,34 & 1333,31 & 1265,93 & 4,91 & 101,32 \\
\hline
\end{tabular}

Tabela 66: Problemas-teste da classe $(10,5,5)$.

\begin{tabular}{c|ccccccc}
\hline Tempo & MIP & LB1 & LB2 & RH1 & RH2 & RH3 & RF1 \\
\hline 060_045 & 7200,12 & 3,09 & 1975,88 & 5764,07 & 5363,36 & 3,78 & 148,45 \\
060_046 & 7200,18 & 3,45 & 4557,48 & 4907,96 & 5887,62 & 5,43 & 199,77 \\
060_047 & 7200,04 & 3,06 & 5633,85 & 1440,06 & 1440,06 & 5,99 & 77,92 \\
060_048 & 7200,05 & 2,31 & 3856,49 & 7200,02 & 3249,39 & 3,18 & 149,01 \\
060_049 & 7200,09 & 3,93 & 3627,58 & 5763,58 & 5796,01 & 6,18 & 268,01 \\
070_045 & 7200,09 & 2,32 & 3032,04 & 5444,87 & 4120,19 & 3,20 & 119,65 \\
070_046 & 7200,02 & 2,40 & 6037,69 & 4527,93 & 4570,61 & 3,10 & 134,38 \\
070_047 & 7200,35 & 2,36 & 6005,96 & 1440,37 & 3652,26 & 2,87 & 154,25 \\
070_048 & 7200,24 & 2,18 & 7200,05 & 5390,68 & 3150,47 & 2,92 & 121,85 \\
070_049 & 7200,34 & 2,20 & 2116,33 & 1440,06 & 1440,06 & 3,10 & 144,63 \\
080_045 & 7200,12 & 2,15 & 4997,93 & 3089,40 & 2663,39 & 2,51 & 101,31 \\
080_046 & 7200,04 & 2,56 & 4483,73 & 4583,08 & 4407,59 & 2,75 & 117,11 \\
080_047 & 7200,13 & 2,37 & 7200,09 & 5277,65 & 3493,72 & 2,68 & 109,87 \\
080_048 & 7200,05 & 1,70 & 1453,93 & 2619,51 & 2557,95 & 2,57 & 113,16 \\
080_049 & 7200,16 & 2,62 & 2067,17 & 5775,20 & 3654,00 & 3,17 & 132,98 \\
\hline
\end{tabular}

Tabela 67: Problemas-teste da classe $(5,10,5)$.

\begin{tabular}{c|ccccccc}
\hline Tempo & MIP & LB1 & LB2 & RH1 & RH2 & RH3 & RF1 \\
\hline 060_050 & 96,50 & 1,47 & 32,65 & 49,89 & 49,92 & 4,77 & 23,01 \\
060_051 & 7200,04 & 1,01 & 257,14 & 215,52 & 297,81 & 1,54 & 25,66 \\
060_052 & 7200,04 & 0,86 & 122,18 & 163,29 & 97,28 & 1,09 & 16,46 \\
060_053 & 7200,01 & 1,22 & 66,82 & 775,03 & 775,12 & 1,09 & 10,45 \\
060_054 & 339,61 & 0,97 & 19,97 & 299,13 & 298,99 & 1,23 & 13,21 \\
070_050 & 558,53 & 1,22 & 155,66 & 288,96 & 289,27 & 1,61 & 12,85 \\
070_051 & 7200,05 & 0,89 & 147,25 & 233,66 & 208,54 & 1,42 & 22,93 \\
070_052 & 4096,63 & 0,64 & 80,73 & 68,17 & 61,18 & 0,98 & 12,95 \\
070_053 & 7200,07 & 0,78 & 62,15 & 249,35 & 295,51 & 1,03 & 16,49 \\
070_054 & 7200,02 & 0,86 & 65,85 & 163,57 & 175,33 & 1,00 & 19,98 \\
080_050 & 7200,05 & 1,26 & 171,13 & 89,51 & 120,62 & 1,51 & 24,02 \\
080_051 & 7200,04 & 0,69 & 129,11 & 184,99 & 180,31 & 0,95 & 16,38 \\
080_052 & 3025,42 & 0,48 & 57,89 & 89,72 & 87,77 & 0,90 & 11,31 \\
080_053 & 7200,05 & 0,66 & 31,75 & 112,41 & 139,42 & 1,14 & 15,72 \\
080_054 & 7200,07 & 0,56 & 63,07 & 70,90 & 86,52 & 0,86 & 16,35 \\
\hline
\end{tabular}

Tabela 68: Problemas-teste da classe $(5,5,10)$.

\begin{tabular}{c|ccccccc}
\hline Tempo & MIP & LB1 & LB2 & RH1 & RH2 & RH3 & RF1 \\
\hline 060_055 & 7200,04 & 0,64 & 7200,05 & 120,85 & 156,87 & 2,61 & 27,85 \\
060_056 & 7200,10 & 0,83 & 7200,05 & 125,92 & 100,53 & 3,10 & 31,50 \\
060_057 & 7200,07 & 0,64 & 4512,13 & 82,60 & 130,49 & 2,22 & 28,55 \\
060_058 & 218,03 & 0,80 & 7200,07 & 94,40 & 94,29 & 0,97 & 4,35 \\
060_059 & 7200,09 & 1,03 & 7200,07 & 200,40 & 128,59 & 1,09 & 6,86 \\
070_055 & 7200,07 & 0,53 & 3130,25 & 71,40 & 80,93 & 2,18 & 27,49 \\
070_056 & 7200,13 & 0,62 & 3976,95 & 101,37 & 69,23 & 2,40 & 27,55 \\
070_057 & 7200,04 & 0,58 & 1839,19 & 73,65 & 76,36 & 1,84 & 22,99 \\
070_058 & 7200,05 & 0,67 & 7200,04 & 19,19 & 57,66 & 2,29 & 24,21 \\
070_059 & 7200,02 & 0,69 & 5911,83 & 64,88 & 71,73 & 2,25 & 26,27 \\
080_055 & 7200,07 & 0,55 & 1312,81 & 61,60 & 47,71 & 1,79 & 24,06 \\
080_056 & 7200,09 & 0,50 & 1507,14 & 86,46 & 58,87 & 1,98 & 24,99 \\
080_057 & 7200,04 & 0,41 & 1837,22 & 61,23 & 46,96 & 1,62 & 21,43 \\
080_058 & 7200,07 & 0,50 & 6721,01 & 69,37 & 36,50 & 1,95 & 22,73 \\
080_059 & 7200,09 & 0,67 & 5898,88 & 43,95 & 55,22 & 1,97 & 22,01 \\
\hline
\end{tabular}


Tabela 69: Problemas-teste da classe $(10,7,7)$.

\begin{tabular}{c|ccccccc}
\hline Tempo & MIP & LB1 & LB2 & RH1 & RH2 & RH3 & RF1 \\
\hline 060_060 & 7200,21 & 16,75 & 7200,23 & 1028,72 & 1028,83 & 37,61 & 595,78 \\
060_061 & 7200,24 & 14,31 & 7200,32 & 1028,76 & 1028,76 & 54,37 & 469,16 \\
060_062 & 7200,13 & 13,14 & 7200,13 & 2057,25 & 2057,39 & 22,50 & 1219,19 \\
060_063 & 7200,18 & 24,43 & 7200,21 & 1028,90 & 1028,67 & 73,29 & 578,53 \\
060_064 & 7200,18 & 10,89 & 7200,16 & 1028,76 & 1028,75 & 36,80 & 1681,91 \\
070_060 & 7200,12 & 14,82 & 7200,29 & 3085,82 & 2057,26 & 17,32 & 1115,98 \\
070_061 & 7200,15 & 12,98 & 7200,10 & 1028,62 & 1028,65 & 13,76 & 868,33 \\
070_062 & 7200,09 & 8,60 & 7200,18 & 5357,29 & 4900,94 & 13,07 & 986,38 \\
070_063 & 7200,09 & 9,58 & 7200,15 & 6192,43 & 2057,28 & 16,99 & 399,52 \\
070_064 & 7200,26 & 7,08 & 7200,18 & 6177,66 & 5254,75 & 14,55 & 1030,14 \\
080_060 & 7200,13 & 12,89 & 7200,21 & 2057,22 & 6173,72 & 11,37 & 738,88 \\
080_061 & 7200,21 & 11,73 & 7200,10 & 6176,06 & 5201,87 & 9,31 & 732,92 \\
080_062 & 7200,43 & 6,72 & 7200,19 & 1028,72 & 1028,65 & 10,84 & 708,43 \\
080_063 & 7200,29 & 14,06 & 7200,21 & 1028,69 & 1028,64 & 11,50 & 904,42 \\
080_064 & 7200,10 & 10,36 & 7200,15 & 1028,72 & 1028,62 & 10,97 & 695,59 \\
\hline
\end{tabular}

Tabela 70: Problemas-teste da classe $(7,10,7)$.

\begin{tabular}{c|ccccccc}
\hline Tempo & MIP & LB1 & LB2 & RH1 & RH2 & RH3 & RF1 \\
\hline 060_065 & 7200,38 & 8,27 & 7200,12 & 2057,20 & 2057,25 & 14,12 & 381,61 \\
060_066 & 7200,12 & 12,21 & 7200,13 & 1028,67 & 1028,72 & 50,68 & 171,62 \\
060_067 & 7200,18 & 6,30 & 7200,07 & 6171,74 & 4737,97 & 13,15 & 397,69 \\
060_068 & 7200,12 & 8,13 & 7200,13 & 1028,64 & 1028,65 & 13,07 & 193,24 \\
060_069 & 7200,16 & 12,99 & 7200,09 & 1028,65 & 1028,72 & 41,89 & 207,54 \\
070_065 & 7200,18 & 6,99 & 7200,09 & 4260,89 & 4930,60 & 10,30 & 348,55 \\
070_066 & 7200,26 & 5,19 & 7200,10 & 5281,10 & 4643,21 & 12,95 & 352,44 \\
070_067 & 7200,12 & 4,34 & 7200,05 & 3732,89 & 3565,37 & 9,27 & 238,93 \\
070_068 & 7200,07 & 5,16 & 7200,16 & 3753,87 & 4544,43 & 10,67 & 383,97 \\
070_069 & 7200,04 & 8,91 & 7200,09 & 1028,69 & 1028,65 & 7,16 & 216,48 \\
080_065 & 7200,13 & 5,16 & 7200,12 & 1028,81 & 2512,94 & 6,79 & 210,91 \\
080_066 & 7200,13 & 5,13 & 7200,15 & 1028,75 & 4385,06 & 7,00 & 262,14 \\
080_067 & 7200,12 & 4,20 & 7200,10 & 2977,61 & 3526,86 & 7,04 & 202,07 \\
080_068 & 7200,15 & 5,88 & 7200,04 & 3486,12 & 4214,69 & 10,62 & 279,35 \\
080_069 & 7200,41 & 6,33 & 7200,12 & 3504,66 & 3634,17 & 12,01 & 357,20 \\
\hline
\end{tabular}

Tabela 71: Problemas-teste da classe $(7,7,10)$.

\begin{tabular}{c|ccccccc}
\hline Tempo & MIP & LB1 & LB2 & RH1 & RH2 & RH3 & RF1 \\
\hline 060_070 & 7200,19 & 7,63 & 7200,12 & 720,12 & 720,15 & 10,83 & 168,12 \\
060_071 & 7200,15 & 4,40 & 7200,12 & 3796,81 & 3839,12 & 11,50 & 379,22 \\
060_072 & 7200,16 & 7,52 & 7200,16 & 2160,07 & 5358,31 & 17,64 & 502,09 \\
060_073 & 7200,13 & 7,47 & 7200,16 & 720,15 & 720,07 & 29,34 & 76,24 \\
060_074 & 7200,09 & 5,52 & 7200,12 & 1440,08 & 3941,72 & 6,65 & 81,82 \\
070_070 & 7200,10 & 4,90 & 7200,07 & 5025,04 & 4352,40 & 14,43 & 570,76 \\
070_071 & 7200,09 & 2,84 & 7200,13 & 2847,49 & 2509,00 & 8,11 & 250,91 \\
070_072 & 7200,13 & 4,38 & 7200,10 & 3593,36 & 3598,82 & 13,88 & 356,34 \\
070_073 & 7200,13 & 5,04 & 7200,19 & 3501,30 & 3900,13 & 9,53 & 365,48 \\
070_074 & 7200,19 & 4,37 & 7200,04 & 3165,93 & 3799,56 & 10,48 & 291,78 \\
080_070 & 7200,18 & 3,88 & 7200,13 & 4358,47 & 3101,44 & 10,48 & 352,34 \\
080_071 & 7200,18 & 2,46 & 7200,10 & 2194,53 & 1560,31 & 6,43 & 207,04 \\
080_072 & 7200,07 & 3,71 & 7200,09 & 4127,77 & 2427,16 & 9,91 & 239,91 \\
080_073 & 7200,04 & 4,62 & 7200,13 & 3323,29 & 2645,71 & 10,00 & 252,08 \\
080_074 & 7200,37 & 3,10 & 7200,10 & 3567,18 & 1866,35 & 9,53 & 233,14 \\
\hline
\end{tabular}

Tabela 72: Problemas-teste da classe $(10,10,10)$.

\begin{tabular}{c|ccccccc}
\hline Tempo & MIP & LB1 & LB2 & RH1 & RH2 & RH3 & RF1 \\
\hline 060_075 & 7200,57 & 86,53 & 7200,30 & 720,32 & 720,30 & 203,58 & 72,24 \\
060_076 & 7200,30 & 125,57 & 7200,21 & 720,27 & 720,18 & 401,16 & 72,29 \\
060_077 & 7200,38 & 88,69 & 7200,30 & 720,43 & 720,30 & 260,27 & 72,40 \\
060_078 & 7200,37 & 100,61 & 7200,21 & 720,24 & 720,23 & 1157,84 & 72,24 \\
060_079 & 7200,49 & 104,21 & 7200,48 & 720,15 & 720,24 & 267,21 & 72,46 \\
070_075 & 7200,35 & 65,74 & 7200,19 & 720,13 & 720,37 & 110,81 & 72,12 \\
070_076 & 7200,77 & 71,11 & 7200,60 & 720,15 & 720,18 & 185,38 & 72,29 \\
070_077 & 7200,27 & 76,53 & 7200,52 & 720,15 & 720,24 & 85,86 & 72,32 \\
070_078 & 7200,52 & 81,84 & 7200,21 & 720,32 & 720,30 & 192,12 & 72,23 \\
070_079 & 7200,19 & 83,51 & 7200,35 & 720,23 & 720,21 & 235,84 & 72,26 \\
080_075 & 7200,37 & 43,20 & 7200,24 & 720,18 & 720,24 & 94,04 & 288,27 \\
080_076 & 7200,41 & 62,03 & 7200,21 & 720,18 & 720,41 & 130,49 & 4118,74 \\
080_077 & 7200,37 & 65,35 & 7200,41 & 6487,27 & 1440,23 & 91,09 & 72,21 \\
080_078 & 7200,32 & 66,44 & 7200,34 & 720,27 & 720,33 & 141,95 & 144,24 \\
080_079 & 7200,37 & 42,60 & 7200,29 & 720,24 & 720,26 & 123,41 & 4735,69 \\
\hline
\end{tabular}

Tabela 73: Problemas-teste da classe $(15,10,10)$.

\begin{tabular}{c|ccccccc}
\hline Tempo & MIP & LB1 & LB2 & RH1 & RH2 & RH3 & RF1 \\
\hline 060_080 & 7201,19 & 635,88 & 7201,05 & 721,02 & 720,93 & 2850,67 & 48,89 \\
060_081 & 7200,96 & 507,03 & 7200,74 & 720,85 & 720,80 & 1468,83 & 49,08 \\
060_082 & 7200,77 & 669,09 & 7200,82 & 720,90 & 720,83 & 1785,54 & 48,67 \\
060_083 & 7200,65 & 400,83 & 7201,04 & 720,93 & 720,88 & 1002,56 & 48,84 \\
060_084 & 7200,88 & 995,46 & 7201,16 & 720,87 & 720,76 & 1651,83 & 49,00 \\
070_080 & 7201,16 & 312,86 & 7201,68 & 720,87 & 720,96 & 1430,03 & 48,83 \\
070_081 & 7201,99 & 267,88 & 7201,10 & 720,66 & 721,04 & 746,62 & 48,78 \\
070_082 & 7201,12 & 331,72 & 7200,88 & 721,04 & 721,26 & 928,75 & 48,84 \\
070_083 & 7200,63 & 349,68 & 7202,55 & 720,79 & 721,02 & 576,36 & 48,73 \\
070_084 & 7201,16 & 363,01 & 7200,79 & 720,69 & 720,68 & 915,90 & 48,80 \\
080_080 & 7201,16 & 254,20 & 7200,99 & 720,83 & 720,87 & 667,84 & 48,75 \\
080_081 & 7202,36 & 229,21 & 7201,12 & 721,29 & 720,68 & 521,28 & 48,80 \\
080_082 & 7200,91 & 270,74 & 7201,21 & 720,72 & 721,13 & 547,08 & 48,94 \\
080_083 & 7201,19 & 878,16 & 7201,02 & 721,32 & 721,75 & 594,47 & 49,02 \\
080_084 & 7200,74 & 219,85 & 7200,80 & 721,02 & 720,69 & 778,77 & 48,91 \\
\hline
\end{tabular}


Tabela 74: Problemas-teste da classe $(10,15,10)$.

\begin{tabular}{c|ccccccc}
\hline Tempo & MIP & LB1 & LB2 & RH1 & RH2 & RH3 & RF1 \\
\hline 060_085 & 7200,34 & 450,56 & 7200,49 & 720,57 & 720,43 & 537,49 & 72,48 \\
060_086 & 7200,63 & 391,09 & 7200,46 & 720,43 & 720,24 & 889,92 & 72,84 \\
060_087 & 7200,41 & 465,09 & 7200,24 & 720,27 & 720,62 & 2389,92 & 72,21 \\
060_088 & 7200,49 & 541,78 & 7200,44 & 720,33 & 720,33 & 967,42 & 72,46 \\
060_089 & 7200,52 & 453,32 & 7200,62 & 720,41 & 720,23 & 824,70 & 72,35 \\
070_085 & 7200,88 & 249,38 & 7200,57 & 720,26 & 720,44 & 759,24 & 72,43 \\
070_086 & 7200,99 & 280,22 & 7200,55 & 720,46 & 720,40 & 555,57 & 72,38 \\
070_087 & 7200,46 & 310,04 & 7200,62 & 720,38 & 720,46 & 936,19 & 72,67 \\
070_088 & 7200,85 & 321,14 & 7200,40 & 720,41 & 720,37 & 556,95 & 72,45 \\
070_089 & 7201,12 & 215,28 & 7200,29 & 720,30 & 720,27 & 690,01 & 72,43 \\
080_085 & 7200,87 & 193,27 & 7200,35 & 720,35 & 720,46 & 415,35 & 72,43 \\
080_086 & 7200,41 & 295,81 & 7200,29 & 720,35 & 720,46 & 437,99 & 72,31 \\
080_087 & 7200,40 & 136,80 & 7200,54 & 720,48 & 720,52 & 458,60 & 72,71 \\
080_088 & 7200,69 & 220,84 & 7200,46 & 720,23 & 720,35 & 486,55 & 72,26 \\
$080 \_089$ & 7200,40 & 229,27 & 7200,46 & 720,29 & 720,41 & 476,27 & 72,35 \\
\hline
\end{tabular}

Tabela 75: Problemas-teste da classe $(10,10,15)$.

\begin{tabular}{c|ccccccc}
\hline Tempo & MIP & LB1 & LB2 & RH1 & RH2 & RH3 & RF1 \\
\hline 060_090 & 7200,54 & 373,23 & 7200,43 & 480,26 & 480,39 & 1496,00 & 48,72 \\
060_091 & 7200,37 & 254,38 & 7200,48 & 480,25 & 480,48 & 463,88 & 48,24 \\
060_092 & 7200,51 & 341,42 & 7200,51 & 480,39 & 480,37 & 646,75 & 48,33 \\
060_093 & 7200,83 & 268,24 & 7200,58 & 480,30 & 480,53 & 591,06 & 48,63 \\
060_094 & 7200,66 & 256,64 & 7200,62 & 480,33 & 480,28 & 1070,60 & 48,70 \\
070_090 & 7201,02 & 216,72 & 7200,74 & 480,39 & 480,41 & 744,75 & 48,53 \\
070_091 & 7200,38 & 130,51 & 7200,37 & 480,33 & 480,30 & 512,82 & 48,36 \\
070_092 & 7200,48 & 219,85 & 7200,48 & 480,42 & 480,20 & 865,93 & 48,52 \\
070_093 & 7200,66 & 116,63 & 7200,48 & 480,64 & 480,45 & 511,09 & 48,45 \\
070_094 & 7200,46 & 157,83 & 7201,43 & 480,22 & 480,42 & 578,20 & 48,72 \\
080_090 & 7200,85 & 178,03 & 7200,49 & 480,30 & 480,25 & 544,58 & 48,30 \\
080_091 & 7200,63 & 112,49 & 7200,41 & 480,48 & 480,36 & 431,51 & 48,44 \\
080_092 & 7200,79 & 147,76 & 7200,35 & 480,36 & 480,41 & 626,47 & 48,36 \\
080_093 & 7200,41 & 109,39 & 7200,63 & 480,33 & 480,28 & 406,06 & 48,45 \\
080_094 & 7200,51 & 91,09 & 7200,43 & 480,39 & 480,30 & 402,50 & 49,06 \\
\hline
\end{tabular}

Tabela 76: Problemas-teste da classe $(15,15,15)$.

\begin{tabular}{c|ccccccc}
\hline Tempo & MIP & LB1 & LB2 & RH1 & RH2 & RH3 & RF1 \\
\hline 060_095 & 7201,80 & 4417,54 & 7201,54 & 482,12 & 482,21 & 7202,07 & 32,00 \\
060_096 & 7201,82 & 7204,36 & 7201,79 & 481,98 & 591,66 & 7203,63 & 32,00 \\
060_097 & 7202,30 & 7202,99 & 7202,05 & 481,90 & 482,20 & 7201,91 & 32,00 \\
060_098 & 7202,32 & 7209,79 & 7201,90 & 482,01 & 481,87 & 7201,79 & 32,00 \\
060_099 & 7202,10 & 7671,52 & 7201,63 & 481,75 & 482,15 & 7202,88 & 32,00 \\
070_095 & 7202,02 & 2789,34 & 7201,91 & 482,15 & 488,81 & 7203,10 & 32,00 \\
070_096 & 7202,35 & 2449,47 & 7201,88 & 481,90 & 481,90 & 6226,12 & 32,00 \\
070_097 & 7202,38 & 4033,41 & 7201,83 & 482,12 & 481,81 & 7202,89 & 32,00 \\
070_098 & 7202,69 & 5746,67 & 7201,80 & 481,70 & 481,89 & 7202,29 & 32,00 \\
070_099 & 7201,90 & 3199,42 & 7201,60 & 482,42 & 482,17 & 7202,82 & 32,00 \\
080_095 & 7200,77 & 1623,35 & 7201,68 & 481,84 & 481,89 & 5949,91 & 32,00 \\
080_096 & 7201,72 & 6719,52 & 7201,88 & 481,87 & 481,50 & 6887,71 & 32,00 \\
080_097 & 7200,58 & 3310,50 & 7201,85 & 481,79 & 481,86 & 7202,96 & 32,00 \\
080_098 & 7202,14 & 2035,70 & 7201,90 & 481,54 & 481,81 & 6808,10 & 32,00 \\
080_099 & 7201,83 & 2007,06 & 7202,19 & 481,54 & 481,68 & 7202,89 & 32,00 \\
\hline
\end{tabular}

Tabela 77: Problemas-teste da classe $(3,3,3)$.

\begin{tabular}{c|cccccccc}
\hline Tempo & AT1 & AT2 & AT3 & AT4 & AT5 & AT6 & ATH1 & ATH2 \\
\hline 060_000 & 4,29 & 7,68 & 13,45 & 15,35 & 3,23 & 5,10 & 5,79 & 23,57 \\
060_001 & 8,44 & 14,63 & 11,37 & 16,54 & 9,55 & 8,53 & 5,48 & 23,59 \\
060_002 & 3,09 & 10,37 & 10,61 & 15,62 & 3,59 & 3,29 & 5,73 & 23,90 \\
060_003 & 6,80 & 5,23 & 12,31 & 15,99 & 7,55 & 6,72 & 5,46 & 20,39 \\
060_004 & 7,75 & 5,49 & 10,70 & 14,66 & 3,81 & 3,43 & 5,60 & 24,01 \\
070_000 & 4,70 & 5,09 & 10,73 & 13,29 & 5,30 & 5,23 & 2,08 & 15,20 \\
070_001 & 9,64 & 7,44 & 25,19 & 12,25 & 6,60 & 9,75 & 1,81 & 14,66 \\
070_002 & 4,03 & 5,20 & 64,46 & 12,90 & 6,44 & 6,97 & 1,92 & 14,62 \\
070_003 & 6,75 & 23,32 & 9,89 & 19,16 & 7,78 & 14,51 & 2,05 & 20,02 \\
070_004 & 10,31 & 5,23 & 38,89 & 11,25 & 5,66 & 6,94 & 2,00 & 15,02 \\
080_000 & 8,55 & 4,82 & 9,33 & 9,92 & 9,08 & 10,06 & 2,00 & 14,80 \\
080_001 & 9,70 & 4,77 & 8,44 & 10,66 & 8,24 & 7,94 & 1,76 & 13,21 \\
080_002 & 9,49 & 9,80 & 10,39 & 12,29 & 5,51 & 9,69 & 1,89 & 14,04 \\
080_003 & 7,82 & 12,00 & 16,82 & 12,17 & 8,31 & 10,08 & 2,03 & 16,10 \\
080_004 & 10,69 & 5,88 & 8,69 & 10,34 & 10,08 & 6,07 & 1,81 & 13,82 \\
\hline
\end{tabular}

Tabela 78: Problemas-teste da classe $(5,3,3)$.

\begin{tabular}{c|cccccccc}
\hline Tempo & AT1 & AT2 & AT3 & AT4 & AT5 & AT6 & ATH1 & ATH2 \\
\hline 060_005 & 13,18 & 14,28 & 12,72 & 17,97 & 6,97 & 10,00 & 3,31 & 20,72 \\
060_006 & 10,66 & 11,83 & 10,23 & 13,17 & 6,49 & 7,80 & 2,96 & 17,00 \\
060_007 & 8,55 & 9,45 & 17,29 & 24,32 & 7,71 & 12,01 & 3,90 & 23,82 \\
060_008 & 10,97 & 12,40 & 16,41 & 20,59 & 7,46 & 8,24 & 4,26 & 28,07 \\
060_009 & 11,79 & 12,04 & 14,62 & 18,77 & 6,24 & 11,16 & 3,42 & 21,68 \\
070_005 & 5,37 & 9,58 & 13,65 & 14,23 & 5,26 & 6,66 & 1,61 & 16,71 \\
070_006 & 8,91 & 17,24 & 10,56 & 13,18 & 10,30 & 13,87 & 1,42 & 14,85 \\
070_007 & 10,61 & 19,34 & 11,72 & 18,10 & 11,97 & 12,12 & 1,58 & 15,82 \\
070_008 & 13,11 & 23,59 & 12,11 & 13,64 & 16,07 & 19,98 & 1,47 & 16,90 \\
070_009 & 9,36 & 29,27 & 11,62 & 17,30 & 6,80 & 8,24 & 1,62 & 16,75 \\
080_005 & 8,16 & 19,86 & 11,45 & 15,55 & 10,44 & 10,94 & 1,50 & 15,21 \\
080_006 & 8,33 & 19,61 & 9,77 & 13,48 & 7,74 & 13,60 & 1,37 & 13,18 \\
080_007 & 10,89 & 11,90 & 13,71 & 24,70 & 9,58 & 11,97 & 1,73 & 16,47 \\
080_008 & 10,42 & 11,26 & 8,28 & 13,65 & 8,10 & 16,65 & 1,26 & 13,09 \\
080_009 & 12,92 & 21,03 & 13,14 & 21,87 & 13,79 & 14,68 & 1,72 & 16,66 \\
\hline
\end{tabular}


Tabela 79: Problemas-teste da classe $(3,5,3)$.

\begin{tabular}{c|cccccccc}
\hline Tempo & AT1 & AT2 & AT3 & AT4 & AT5 & AT6 & ATH1 & ATH2 \\
\hline 060_010 & 4,46 & 24,73 & 10,36 & 16,36 & 7,39 & 18,99 & 8,46 & 23,98 \\
060_011 & 5,68 & 23,49 & 7,96 & 14,12 & 8,58 & 17,00 & 7,89 & 21,00 \\
060_012 & 5,23 & 8,94 & 11,33 & 12,29 & 6,12 & 7,89 & 9,10 & 24,30 \\
060_013 & 8,02 & 12,09 & 10,44 & 14,45 & 6,69 & 11,65 & 7,94 & 22,76 \\
060_014 & 9,05 & 8,61 & 8,66 & 13,14 & 9,28 & 9,39 & 8,80 & 24,68 \\
$070 \_010$ & 6,85 & 15,12 & 8,46 & 11,86 & 3,38 & 5,24 & 2,40 & 17,19 \\
$070 \_011$ & 7,30 & 18,25 & 8,78 & 12,96 & 5,60 & 12,79 & 2,09 & 15,15 \\
070_012 & 9,50 & 20,64 & 8,69 & 11,43 & 10,84 & 9,72 & 3,14 & 26,58 \\
070_013 & 6,93 & 7,39 & 10,08 & 13,73 & 11,20 & 11,76 & 2,45 & 17,38 \\
$070 \_014$ & 13,06 & 11,36 & 8,16 & 17,05 & 11,78 & 14,60 & 2,34 & 16,51 \\
$080 \_010$ & 10,55 & 15,79 & 9,36 & 11,14 & 11,37 & 14,21 & 2,54 & 15,30 \\
$080 \_011$ & 3,56 & 4,57 & 10,75 & 12,57 & 3,34 & 4,54 & 2,26 & 16,22 \\
$080 \_012$ & 11,71 & 14,87 & 10,67 & 10,64 & 5,80 & 16,71 & 3,23 & 26,71 \\
$080 \_013$ & 10,20 & 12,79 & 10,84 & 11,81 & 13,10 & 12,45 & 2,39 & 16,63 \\
$080 \_014$ & 13,07 & 8,53 & 9,53 & 16,50 & 10,94 & 10,14 & 2,39 & 15,19 \\
\hline
\end{tabular}

Tabela 80: Problemas-teste da classe $(3,3,5)$.

\begin{tabular}{c|cccccccc}
\hline Tempo & AT1 & AT2 & AT3 & AT4 & AT5 & AT6 & ATH1 & ATH2 \\
\hline 060_015 & 9,63 & 19,02 & 18,78 & 26,77 & 10,72 & 14,57 & 4,49 & 28,63 \\
060_016 & 14,15 & 16,76 & 14,84 & 19,48 & 11,89 & 14,46 & 4,10 & 23,14 \\
060_017 & 9,44 & 14,98 & 19,10 & 23,98 & 12,11 & 8,00 & 4,59 & 30,36 \\
060_018 & 10,78 & 8,89 & 17,35 & 17,43 & 5,76 & 7,00 & 7,52 & 27,49 \\
060_019 & 14,69 & 13,82 & 18,67 & 20,44 & 16,04 & 8,03 & 5,90 & 33,52 \\
$070 \_015$ & 14,77 & 16,51 & 17,02 & 19,98 & 7,50 & 10,95 & 2,03 & 23,49 \\
070_016 & 17,80 & 19,05 & 17,94 & 19,64 & 17,11 & 16,99 & 2,01 & 21,58 \\
070_017 & 10,26 & 6,72 & 17,66 & 25,01 & 6,10 & 9,50 & 2,51 & 24,51 \\
$070 \_018$ & 23,46 & 17,97 & 17,88 & 18,14 & 17,05 & 15,87 & 2,59 & 19,86 \\
$070 \_019$ & 6,49 & 6,57 & 22,04 & 22,81 & 7,25 & 6,33 & 2,56 & 29,20 \\
$080 \_015$ & 16,91 & 10,84 & 16,90 & 20,62 & 12,06 & 17,08 & 1,86 & 20,65 \\
$080 \_016$ & 11,40 & 19,87 & 15,88 & 18,02 & 7,33 & 6,12 & 1,94 & 19,36 \\
$080 \_017$ & 8,03 & 13,07 & 14,66 & 17,57 & 4,65 & 9,19 & 2,03 & 21,06 \\
$080 \_018$ & 19,56 & 18,88 & 23,14 & 16,69 & 23,63 & 22,25 & 2,67 & 20,58 \\
$080 \_019$ & 13,29 & 11,59 & 18,22 & 18,33 & 10,08 & 10,92 & 2,18 & 22,34 \\
\hline
\end{tabular}

Tabela 81: Problemas-teste da classe $(5,5,5)$.

\begin{tabular}{c|cccccccc}
\hline Tempo & AT1 & AT2 & AT3 & AT4 & AT5 & AT6 & ATH1 & ATH2 \\
\hline 060_020 & 24,13 & 38,89 & 26,07 & 40,89 & 13,84 & 39,14 & 5,26 & 54,85 \\
060_021 & 23,51 & 69,30 & 19,55 & 29,48 & 17,07 & 29,69 & 4,56 & 34,85 \\
060_022 & 16,55 & 35,69 & 24,52 & 38,86 & 17,02 & 29,11 & 4,68 & 46,51 \\
060_023 & 26,41 & 27,19 & 25,16 & 35,51 & 29,16 & 23,63 & 5,04 & 112,84 \\
060_024 & 38,72 & 36,80 & 34,23 & 42,32 & 33,43 & 35,80 & 5,70 & 117,70 \\
070_020 & 17,21 & 39,98 & 29,73 & 43,06 & 11,64 & 61,65 & 3,81 & 50,50 \\
070_021 & 26,72 & 63,66 & 24,73 & 27,69 & 41,73 & 33,03 & 3,25 & 28,47 \\
070_022 & 21,79 & 42,34 & 21,61 & 30,50 & 20,98 & 49,84 & 3,01 & 31,65 \\
070_023 & 16,61 & 38,77 & 29,58 & 31,84 & 15,85 & 18,89 & 3,42 & 88,84 \\
070_024 & 15,21 & 37,97 & 36,85 & 40,76 & 15,88 & 37,11 & 4,28 & 159,65 \\
080_020 & 22,54 & 47,53 & 19,78 & 31,87 & 20,36 & 22,43 & 4,57 & 32,78 \\
080_021 & 19,77 & 25,44 & 17,29 & 23,38 & 25,40 & 50,39 & 3,00 & 23,12 \\
080_022 & 24,90 & 45,96 & 19,67 & 29,30 & 29,34 & 39,20 & 3,10 & 29,25 \\
080_023 & 28,94 & 23,07 & 23,14 & 26,15 & 16,38 & 27,69 & 3,76 & 31,23 \\
080_024 & 37,72 & 85,83 & 39,90 & 27,52 & 14,29 & 28,02 & 3,90 & 32,28 \\
\hline
\end{tabular}

Tabela 82: Problemas-teste da classe $(7,5,5)$.

\begin{tabular}{c|cccccccc}
\hline Tempo & AT1 & AT2 & AT3 & AT4 & AT5 & AT6 & ATH1 & ATH2 \\
\hline 060_025 & 20,20 & 51,78 & 36,85 & 37,74 & 40,28 & 88,95 & 6,01 & 45,55 \\
060_026 & 22,42 & 102,98 & 48,42 & 54,60 & 36,65 & 24,27 & 6,62 & 64,09 \\
060_027 & 21,95 & 70,45 & 32,29 & 45,16 & 22,12 & 28,91 & 8,77 & 240,69 \\
060_028 & 32,49 & 39,03 & 34,26 & 45,10 & 24,13 & 94,88 & 5,77 & 39,73 \\
060_029 & 39,52 & 142,12 & 49,40 & 47,49 & 31,96 & 83,88 & 7,21 & 146,17 \\
070_025 & 54,90 & 111,01 & 25,02 & 52,46 & 42,01 & 78,00 & 4,70 & 37,38 \\
070_026 & 33,93 & 63,12 & 30,20 & 39,06 & 43,74 & 107,20 & 4,42 & 41,14 \\
070_027 & 45,32 & 41,70 & 23,48 & 34,66 & 37,81 & 77,44 & 4,37 & 35,35 \\
070_028 & 36,88 & 62,95 & 20,83 & 37,74 & 32,68 & 111,85 & 4,32 & 36,93 \\
070_029 & 32,40 & 87,13 & 26,54 & 36,74 & 16,10 & 55,88 & 4,77 & 38,95 \\
080_025 & 41,68 & 57,95 & 24,63 & 32,76 & 26,41 & 71,93 & 4,32 & 32,12 \\
080_026 & 40,90 & 148,17 & 26,89 & 33,81 & 19,94 & 109,20 & 4,40 & 34,74 \\
080_027 & 21,20 & 76,35 & 24,68 & 35,74 & 44,15 & 74,04 & 3,98 & 31,47 \\
080_028 & 71,40 & 74,51 & 19,10 & 32,53 & 37,47 & 64,01 & 4,54 & 32,31 \\
080_029 & 47,92 & 47,88 & 23,32 & 39,73 & 41,82 & 139,11 & 4,31 & 32,82 \\
\hline
\end{tabular}

Tabela 83: Problemas-teste da classe $(5,7,5)$.

\begin{tabular}{c|cccccccc}
\hline Tempo & AT1 & AT2 & AT3 & AT4 & AT5 & AT6 & ATH1 & ATH2 \\
\hline 060_030 & 37,67 & 58,49 & 39,36 & 46,14 & 19,70 & 41,57 & 9,42 & 120,65 \\
060_031 & 18,39 & 51,12 & 31,14 & 42,70 & 20,75 & 46,99 & 8,61 & 263,73 \\
060_032 & 28,94 & 93,87 & 30,53 & 33,39 & 27,27 & 64,10 & 5,68 & 36,07 \\
060_033 & 30,30 & 41,36 & 27,44 & 42,04 & 17,25 & 87,13 & 8,00 & 39,45 \\
060_034 & 40,72 & 30,39 & 36,71 & 46,04 & 25,71 & 66,50 & 7,41 & 127,06 \\
070_030 & 40,86 & 96,30 & 23,12 & 37,61 & 24,09 & 52,37 & 4,81 & 42,90 \\
070_031 & 49,33 & 93,83 & 35,96 & 42,23 & 21,58 & 107,83 & 4,65 & 123,76 \\
070_032 & 22,03 & 31,09 & 23,70 & 26,46 & 22,37 & 42,82 & 3,78 & 33,52 \\
070_033 & 41,22 & 52,18 & 21,03 & 36,22 & 21,43 & 70,86 & 3,96 & 34,40 \\
070_034 & 26,29 & 85,05 & 28,59 & 48,75 & 22,25 & 61,84 & 7,13 & 96,60 \\
080_030 & 42,21 & 91,68 & 20,42 & 31,43 & 55,02 & 43,56 & 4,31 & 36,27 \\
080_031 & 45,90 & 108,75 & 27,11 & 29,80 & 20,16 & 73,60 & 4,43 & 31,32 \\
080_032 & 27,78 & 47,39 & 32,96 & 36,13 & 25,37 & 48,66 & 3,85 & 34,05 \\
080_033 & 36,99 & 50,67 & 33,49 & 27,22 & 41,84 & 40,61 & 4,04 & 33,46 \\
080_034 & 43,99 & 111,34 & 36,05 & 40,22 & 35,90 & 99,84 & 4,59 & 38,17 \\
\hline
\end{tabular}


Tabela 84: Problemas-teste da classe $(5,5,7)$.

\begin{tabular}{c|cccccccc}
\hline Tempo & AT1 & AT2 & AT3 & AT4 & AT5 & AT6 & ATH1 & ATH2 \\
\hline 060_035 & 19,27 & 102,79 & 67,64 & 69,09 & 44,43 & 41,11 & 7,18 & 157,56 \\
060_036 & 25,26 & 53,62 & 49,03 & 51,50 & 15,41 & 24,51 & 10,26 & 113,35 \\
060_037 & 41,20 & 82,84 & 53,13 & 56,89 & 33,93 & 45,15 & 7,33 & 281,29 \\
060_038 & 21,78 & 90,36 & 49,17 & 58,88 & 36,88 & 49,34 & 6,44 & 146,02 \\
060_039 & 25,43 & 58,80 & 40,53 & 54,69 & 29,53 & 52,59 & 8,22 & 99,12 \\
070_035 & 53,88 & 41,12 & 42,06 & 42,81 & 36,41 & 31,71 & 4,35 & 44,54 \\
070_036 & 22,43 & 47,83 & 47,30 & 56,99 & 22,14 & 24,34 & 4,80 & 48,67 \\
070_037 & 20,69 & 46,74 & 52,31 & 60,92 & 26,71 & 68,24 & 5,16 & 114,69 \\
070_038 & 37,99 & 89,95 & 29,25 & 41,68 & 26,36 & 31,22 & 4,88 & 45,40 \\
070_039 & 28,03 & 67,46 & 29,78 & 44,09 & 29,53 & 53,26 & 4,71 & 41,67 \\
080_035 & 35,55 & 69,80 & 35,76 & 47,56 & 27,94 & 33,65 & 4,31 & 40,19 \\
080_036 & 44,71 & 46,25 & 29,36 & 40,79 & 37,80 & 61,42 & 4,49 & 38,83 \\
080_037 & 28,28 & 82,63 & 44,76 & 50,06 & 46,18 & 43,45 & 5,05 & 44,32 \\
080_038 & 23,84 & 87,38 & 27,81 & 39,67 & 39,48 & 55,10 & 4,96 & 40,39 \\
080_039 & 42,11 & 59,17 & 29,38 & 42,21 & 33,21 & 51,61 & 4,26 & 34,29 \\
\hline
\end{tabular}

Tabela 85: Problemas-teste da classe $(7,7,7)$.

\begin{tabular}{c|cccccccc}
\hline Tempo & AT1 & AT2 & AT3 & AT4 & AT5 & AT6 & ATH1 & ATH2 \\
060_040 & 220,15 & 69,89 & 102,12 & 64,30 & 173,41 & 9,33 & 103,04 & \\
060_041 & 76,55 & 97,69 & 86,53 & 31,01 & 150,18 & 11,75 & 205,44 & \\
060_042 & 89,31 & 49,39 & 74,05 & 52,53 & 74,23 & 10,25 & 157,28 & \\
060_043 & 269,38 & 77,10 & 101,92 & 50,75 & 160,31 & 9,70 & 260,57 & \\
060_044 & 152,82 & 53,43 & 103,02 & 34,40 & 194,78 & 11,39 & 113,37 & \\
070_040 & 263,86 & 45,16 & 64,18 & 62,45 & 211,23 & 10,42 & 56,57 & \\
070_041 & 157,16 & 51,64 & 74,96 & 54,02 & 86,70 & 8,74 & 72,09 & \\
070_042 & 194,22 & 43,06 & 60,11 & 69,11 & 126,06 & 7,11 & 53,40 & \\
070_043 & 145,83 & 49,02 & 72,24 & 67,46 & 127,56 & 8,86 & 62,68 & \\
070_044 & 168,08 & 48,80 & 78,97 & 61,59 & 110,21 & 8,83 & 64,82 & \\
080_040 & 266,42 & 37,30 & 60,84 & 57,24 & 73,49 & 7,83 & 55,10 & \\
080_041 & 172,49 & 51,48 & 56,75 & 45,93 & 143,04 & 8,19 & 57,14 & \\
080_042 & 143,83 & 43,62 & 51,90 & 62,11 & 136,72 & 7,15 & 52,60 & \\
080_043 & 134,85 & 38,02 & 52,07 & 54,69 & 159,04 & 7,85 & 53,06 & \\
080_044 & 304,80 & 39,22 & 62,82 & 70,48 & 277,39 & 7,94 & 52,32 & \\
\hline
\end{tabular}

Tabela 86: Problemas-teste da classe $(10,5,5)$.

\begin{tabular}{c|cccccccc}
\hline Tempo & AT1 & AT2 & AT3 & AT4 & AT5 & AT6 & ATH1 & ATH2 \\
\hline 060_045 & 63,73 & 59,19 & 59,56 & 78,05 & 33,32 & 60,29 & 18,11 & 301,25 \\
060_046 & 22,76 & 107,19 & 57,21 & 72,26 & 52,81 & 63,31 & 13,11 & 230,94 \\
060_047 & 45,32 & 37,39 & 93,54 & 82,84 & 40,19 & 64,16 & 8,74 & 59,65 \\
060_048 & 65,37 & 100,25 & 43,90 & 91,82 & 73,70 & 65,77 & 7,85 & 50,39 \\
060_049 & 42,89 & 96,00 & 50,18 & 84,19 & 43,91 & 78,28 & 11,42 & 270,12 \\
070_045 & 33,49 & 258,62 & 36,24 & 47,39 & 54,48 & 76,52 & 5,93 & 47,14 \\
070_046 & 45,44 & 79,28 & 38,34 & 53,26 & 68,97 & 156,31 & 8,68 & 120,85 \\
070_047 & 27,19 & 53,70 & 34,28 & 46,02 & 46,27 & 74,07 & 7,52 & 47,07 \\
070_048 & 58,08 & 114,77 & 32,87 & 48,47 & 62,53 & 39,75 & 6,35 & 45,38 \\
070_049 & 68,35 & 105,80 & 51,46 & 59,11 & 55,30 & 179,42 & 7,41 & 64,83 \\
080_045 & 121,76 & 116,22 & 29,38 & 42,17 & 107,33 & 167,78 & 6,30 & 43,57 \\
080_046 & 66,69 & 224,84 & 34,71 & 43,57 & 73,09 & 191,90 & 6,15 & 45,66 \\
080_047 & 73,26 & 228,84 & 29,48 & 43,43 & 56,94 & 90,39 & 6,10 & 42,04 \\
080_048 & 67,52 & 268,18 & 31,44 & 47,84 & 36,52 & 70,75 & 6,63 & 49,03 \\
080_049 & 54,48 & 169,26 & 30,59 & 47,78 & 66,93 & 123,04 & 5,82 & 47,75 \\
\hline
\end{tabular}

Tabela 87: Problemas-teste da classe $(5,10,5)$.

\begin{tabular}{c|cccccccc}
\hline Tempo & AT1 & AT2 & AT3 & AT4 & AT5 & AT6 & ATH1 & ATH2 \\
060_050 & 46,02 & 43,63 & 46,10 & 42,98 & 41,47 & 40,34 & 17,77 & 57,08 \\
060_051 & 43,77 & 208,67 & 28,59 & 49,66 & 34,27 & 27,10 & 11,08 & 145,42 \\
060_052 & 37,33 & 131,85 & 23,92 & 32,75 & 25,21 & 129,25 & 9,28 & 36,68 \\
060_053 & 28,27 & 90,03 & 34,24 & 42,37 & 22,45 & 102,15 & 13,34 & 53,88 \\
060_054 & 36,83 & 260,96 & 27,22 & 43,63 & 42,23 & 131,07 & 8,13 & 44,30 \\
070_050 & 69,73 & 297,17 & 35,38 & 44,33 & 48,80 & 381,77 & 8,52 & 71,76 \\
070_051 & 56,25 & 232,79 & 26,60 & 37,22 & 31,98 & 237,61 & 8,39 & 85,65 \\
070_052 & 31,95 & 279,56 & 21,09 & 26,90 & 30,50 & 185,89 & 4,74 & 40,33 \\
070_053 & 56,69 & 216,25 & 27,33 & 34,29 & 31,06 & 90,28 & 5,18 & 42,59 \\
070_054 & 54,54 & 82,82 & 24,29 & 32,32 & 46,80 & 124,74 & 4,77 & 40,06 \\
080_050 & 46,83 & 211,83 & 25,65 & 37,39 & 46,75 & 114,44 & 5,93 & 44,48 \\
080_051 & 38,24 & 466,96 & 22,73 & 36,04 & 36,49 & 289,79 & 5,38 & 59,67 \\
080_052 & 33,15 & 126,24 & 20,22 & 33,18 & 27,07 & 214,00 & 5,18 & 37,36 \\
080_053 & 43,74 & 100,26 & 29,20 & 36,12 & 22,75 & 87,95 & 5,21 & 39,69 \\
080_054 & 43,56 & 57,99 & 22,21 & 34,91 & 31,68 & 84,66 & 4,73 & 42,67 \\
\hline
\end{tabular}

Tabela 88: Problemas-teste da classe $(5,5,10)$.

\begin{tabular}{c|cccccccc}
\hline Tempo & AT1 & AT2 & AT3 & AT4 & AT5 & AT6 & ATH1 & ATH2 \\
\hline 060_055 & 34,10 & 106,63 & 77,11 & 87,08 & 36,21 & 63,98 & 10,44 & 66,13 \\
060_056 & 45,66 & 85,29 & 48,00 & 102,71 & 41,76 & 74,87 & 39,11 & 96,32 \\
060_057 & 29,08 & 43,46 & 64,12 & 91,53 & 27,71 & 57,07 & 12,15 & 85,78 \\
060_058 & 54,34 & 41,31 & 66,88 & 92,07 & 31,61 & 30,50 & 54,73 & 84,37 \\
060_059 & 41,43 & 137,66 & 67,27 & 84,22 & 45,97 & 103,88 & 40,05 & 95,43 \\
070_055 & 40,76 & 167,30 & 62,70 & 65,41 & 29,94 & 97,20 & 8,39 & 67,33 \\
070_056 & 45,52 & 119,58 & 77,11 & 120,14 & 40,42 & 72,42 & 10,16 & 87,95 \\
070_057 & 32,45 & 54,23 & 67,38 & 74,97 & 33,23 & 47,16 & 7,77 & 70,37 \\
070_058 & 51,03 & 70,31 & 78,53 & 76,58 & 33,95 & 61,39 & 12,18 & 66,07 \\
070_059 & 52,53 & 97,81 & 78,80 & 103,46 & 44,63 & 106,60 & 12,04 & 103,71 \\
080_055 & 33,01 & 92,76 & 70,50 & 57,39 & 34,82 & 109,89 & 7,22 & 51,89 \\
080_056 & 53,35 & 95,66 & 58,47 & 78,33 & 31,26 & 61,23 & 8,05 & 68,19 \\
080_057 & 49,66 & 100,06 & 52,53 & 60,76 & 46,54 & 65,15 & 9,28 & 64,44 \\
080_058 & 92,73 & 88,44 & 46,83 & 61,42 & 40,11 & 64,16 & 11,47 & 58,64 \\
080_059 & 50,25 & 94,19 & 57,89 & 66,38 & 40,17 & 180,00 & 9,94 & 58,05 \\
\hline
\end{tabular}


Tabela 89: Problemas-teste da classe $(10,7,7)$.

\begin{tabular}{c|cccccccc}
\hline Tempo & AT1 & AT2 & AT3 & AT4 & AT5 & AT6 & ATH1 & ATH2 \\
\hline 060_060 & 122,07 & 142,13 & 81,48 & 131,82 & 78,75 & 180,38 & 14,95 & 146,17 \\
060_061 & 122,34 & 240,88 & 74,62 & 134,10 & 62,28 & 112,71 & 16,86 & 124,43 \\
060_062 & 71,57 & 228,95 & 78,77 & 117,61 & 91,07 & 165,00 & 15,63 & 105,07 \\
060_063 & 80,39 & 241,66 & 84,10 & 123,12 & 95,13 & 227,73 & 16,80 & 238,12 \\
060_064 & 99,50 & 218,99 & 83,13 & 120,37 & 49,75 & 243,80 & 16,40 & 170,49 \\
070_060 & 84,18 & 324,61 & 71,28 & 90,48 & 63,80 & 105,69 & 11,87 & 87,81 \\
070_061 & 151,77 & 275,87 & 66,13 & 89,62 & 110,17 & 197,33 & 16,04 & 89,95 \\
070_062 & 70,92 & 187,00 & 64,23 & 90,61 & 73,77 & 176,36 & 11,45 & 83,60 \\
070_063 & 102,80 & 153,97 & 66,91 & 93,17 & 115,00 & 88,42 & 15,07 & 153,90 \\
070_064 & 121,35 & 222,21 & 63,09 & 82,96 & 120,35 & 214,27 & 11,97 & 89,33 \\
080_060 & 89,58 & 629,96 & 61,79 & 80,36 & 74,69 & 302,52 & 12,06 & 89,59 \\
080_061 & 153,49 & 420,63 & 67,47 & 82,12 & 161,96 & 287,84 & 13,35 & 83,52 \\
080_062 & 83,80 & 334,23 & 53,20 & 89,27 & 124,02 & 303,78 & 12,90 & 84,08 \\
080_063 & 107,39 & 391,86 & 58,17 & 71,78 & 77,70 & 340,29 & 15,83 & 89,23 \\
080_064 & 194,00 & 361,45 & 68,30 & 76,55 & 94,46 & 276,62 & 11,69 & 82,74 \\
\hline
\end{tabular}

Tabela 90: Problemas-teste da classe $(7,10,7)$.

\begin{tabular}{c|cccccccc}
\hline Tempo & AT1 & AT2 & AT3 & AT4 & AT5 & AT6 & ATH1 & ATH2 \\
\hline 060_065 & 72,57 & 176,73 & 59,73 & 91,87 & 49,45 & 84,29 & 16,61 & 240,37 \\
060_066 & 84,80 & 369,60 & 70,82 & 98,37 & 75,33 & 231,65 & 21,51 & 121,46 \\
060_067 & 106,27 & 185,59 & 70,33 & 92,96 & 72,98 & 379,04 & 17,36 & 90,34 \\
060_068 & 75,30 & 367,93 & 60,54 & 101,15 & 102,15 & 238,90 & 14,87 & 163,27 \\
060_069 & 83,31 & 206,22 & 62,57 & 105,05 & 97,86 & 204,17 & 15,84 & 136,22 \\
070_065 & 138,64 & 333,16 & 60,87 & 143,97 & 53,80 & 283,58 & 10,59 & 129,65 \\
070_066 & 80,97 & 156,30 & 55,04 & 75,61 & 62,14 & 88,92 & 24,43 & 81,06 \\
070_067 & 92,54 & 730,18 & 44,99 & 69,37 & 59,36 & 245,16 & 10,95 & 67,84 \\
070_068 & 76,61 & 304,17 & 56,77 & 67,89 & 57,14 & 317,88 & 13,53 & 103,13 \\
070_069 & 82,15 & 78,67 & 70,51 & 108,75 & 67,33 & 331,50 & 11,70 & 171,41 \\
080_065 & 136,22 & 359,85 & 54,91 & 70,95 & 105,02 & 185,47 & 10,36 & 76,99 \\
080_066 & 152,88 & 124,05 & 46,60 & 68,00 & 78,45 & 265,81 & 11,56 & 78,84 \\
080_067 & 101,56 & 208,04 & 35,94 & 55,78 & 104,32 & 211,27 & 9,59 & 65,71 \\
080_068 & 99,79 & 61,67 & 38,25 & 67,00 & 80,75 & 244,66 & 9,89 & 65,75 \\
080_069 & 121,06 & 469,28 & 62,35 & 125,71 & 83,41 & 512,57 & 10,00 & 75,58 \\
\hline
\end{tabular}

Tabela 91: Problemas-teste da classe $(7,7,10)$.

\begin{tabular}{c|cccccccc}
\hline Tempo & AT1 & AT2 & AT3 & AT4 & AT5 & AT6 & ATH1 & ATH2 \\
\hline 060_070 & 197,11 & 283,95 & 80,40 & 143,44 & 74,27 & 432,18 & 17,77 & 199,65 \\
060_071 & 71,89 & 135,04 & 78,31 & 155,57 & 64,72 & 205,69 & 14,91 & 246,06 \\
060_072 & 142,21 & 260,49 & 76,49 & 143,24 & 62,43 & 161,59 & 16,94 & 159,78 \\
060_073 & 70,45 & 266,90 & 72,34 & 128,14 & 108,53 & 327,49 & 18,60 & 253,28 \\
060_074 & 78,48 & 362,08 & 71,46 & 115,53 & 92,01 & 173,05 & 19,61 & 204,28 \\
070_070 & 96,80 & 236,40 & 83,62 & 98,31 & 58,44 & 205,91 & 15,37 & 132,90 \\
070_071 & 111,79 & 119,04 & 58,31 & 77,70 & 123,37 & 333,36 & 12,86 & 88,66 \\
070_072 & 99,17 & 265,12 & 65,22 & 119,82 & 82,13 & 105,39 & 16,88 & 94,46 \\
070_073 & 74,99 & 128,41 & 69,79 & 104,60 & 68,94 & 220,20 & 15,07 & 172,74 \\
070_074 & 58,11 & 193,49 & 68,13 & 94,63 & 52,85 & 110,71 & 14,98 & 107,50 \\
080_070 & 84,08 & 204,13 & 72,32 & 105,58 & 59,22 & 155,14 & 15,19 & 109,84 \\
080_071 & 140,51 & 266,67 & 51,42 & 74,91 & 98,31 & 89,53 & 14,07 & 78,13 \\
080_072 & 133,99 & 370,36 & 56,41 & 95,08 & 59,53 & 142,04 & 12,00 & 84,33 \\
080_073 & 107,91 & 249,85 & 61,00 & 85,49 & 78,20 & 197,42 & 20,98 & 92,18 \\
080_074 & 156,92 & 154,49 & 60,70 & 85,83 & 78,56 & 143,38 & 12,62 & 91,79 \\
\hline
\end{tabular}

Tabela 92: Problemas-teste da classe $(10,10,10)$.

\begin{tabular}{c|cccccccc}
\hline Tempo & AT1 & AT2 & AT3 & AT4 & AT5 & AT6 & ATH1 & ATH2 \\
\hline 060_075 & 120,01 & 480,61 & 108,61 & 188,61 & 238,40 & 512,74 & 37,68 & 242,79 \\
060_076 & 139,15 & 388,30 & 116,52 & 198,20 & 101,90 & 567,36 & 51,09 & 303,06 \\
060_077 & 237,28 & 493,24 & 117,33 & 202,40 & 139,68 & 583,66 & 44,35 & 327,31 \\
060_078 & 163,26 & 880,94 & 123,07 & 210,48 & 169,22 & 1137,73 & 58,11 & 305,78 \\
060_079 & 93,91 & 618,00 & 117,62 & 192,13 & 254,64 & 172,54 & 53,01 & 362,59 \\
070_075 & 251,22 & 468,33 & 122,04 & 194,71 & 167,16 & 303,69 & 31,08 & 158,37 \\
070_076 & 213,47 & 361,16 & 100,23 & 191,98 & 167,28 & 373,72 & 37,77 & 205,41 \\
070_077 & 157,62 & 578,26 & 115,77 & 192,58 & 165,42 & 739,04 & 31,56 & 191,74 \\
070_078 & 182,09 & 1111,07 & 107,73 & 208,03 & 211,21 & 344,50 & 38,61 & 226,54 \\
070_079 & 156,61 & 909,14 & 105,11 & 188,39 & 151,04 & 137,48 & 35,37 & 209,88 \\
080_075 & 191,34 & 1102,10 & 84,33 & 143,69 & 130,68 & 979,02 & 23,18 & 145,74 \\
080_076 & 337,65 & 958,39 & 91,31 & 138,08 & 231,69 & 846,24 & 31,57 & 168,70 \\
080_077 & 268,56 & 433,95 & 83,56 & 142,41 & 252,46 & 769,37 & 24,26 & 163,24 \\
080_078 & 243,55 & 983,12 & 88,75 & 148,43 & 146,08 & 1422,12 & 31,33 & 172,30 \\
080_079 & 175,69 & 997,39 & 88,30 & 139,47 & 327,74 & 283,63 & 28,55 & 162,15 \\
\hline
\end{tabular}


Tabela 93: Problemas-teste da classe $(15,10,10)$.

\begin{tabular}{c|cccccccc}
\hline Tempo & AT1 & AT2 & AT3 & AT4 & AT5 & AT6 & ATH1 & ATH2 \\
\hline 060_080 & 314,64 & 1201,83 & 190,60 & 317,43 & 527,24 & 801,28 & 117,17 & 520,09 \\
060_081 & 229,40 & 790,66 & 176,05 & 312,50 & 265,03 & 1918,89 & 86,74 & 379,55 \\
060_082 & 158,89 & 735,31 & 181,98 & 305,53 & 161,96 & 2487,98 & 101,20 & 383,43 \\
060_083 & 342,19 & 1660,59 & 157,14 & 244,94 & 329,04 & 1145,86 & 67,53 & 305,79 \\
060_084 & 246,01 & 572,35 & 170,98 & 280,62 & 222,18 & 1119,99 & 94,88 & 412,81 \\
070_080 & 429,16 & 707,20 & 160,38 & 244,92 & 328,52 & 1130,43 & 69,70 & 353,79 \\
070_081 & 432,48 & 704,92 & 145,74 & 233,49 & 458,78 & 1288,91 & 61,00 & 321,71 \\
070_082 & 272,85 & 1081,51 & 155,07 & 233,91 & 274,28 & 1298,24 & 63,96 & 367,51 \\
070_083 & 297,85 & 1112,19 & 133,87 & 212,24 & 284,13 & 1635,50 & 50,87 & 273,81 \\
070_084 & 370,89 & 2486,80 & 147,61 & 235,01 & 274,02 & 1126,80 & 68,55 & 324,62 \\
080_080 & 531,96 & 1645,98 & 131,51 & 222,82 & 454,56 & 806,14 & 53,46 & 261,99 \\
080_081 & 547,69 & 2385,40 & 128,69 & 209,62 & 443,33 & 1212,91 & 49,20 & 286,73 \\
080_082 & 469,56 & 856,07 & 126,38 & 198,51 & 228,35 & 1133,08 & 49,31 & 267,00 \\
080_083 & 234,13 & 1901,23 & 124,47 & 194,24 & 351,03 & 1583,05 & 45,26 & 240,41 \\
080_084 & 630,04 & 1068,97 & 129,37 & 207,98 & 339,15 & 1030,48 & 49,31 & 242,08 \\
\hline
\end{tabular}

Tabela 94: Problemas-teste da classe $(10,15,10)$.

\begin{tabular}{c|cccccccc}
\hline Tempo & AT1 & AT2 & AT3 & AT4 & AT5 & AT6 & ATH1 & ATH2 \\
\hline 060_085 & 228,26 & 1259,65 & 175,20 & 250,41 & 270,91 & 517,92 & 133,60 & 440,73 \\
060_086 & 202,52 & 737,09 & 152,87 & 226,23 & 184,64 & 768,51 & 106,72 & 385,35 \\
060_087 & 194,67 & 1584,99 & 172,96 & 252,54 & 192,13 & 1067,33 & 189,62 & 648,20 \\
060_088 & 261,01 & 753,88 & 195,72 & 222,79 & 221,09 & 1019,67 & 152,99 & 579,01 \\
060_089 & 319,12 & 297,45 & 346,03 & 324,31 & 300,76 & 289,51 & 175,84 & 853,06 \\
070_085 & 328,66 & 1487,27 & 119,37 & 191,16 & 234,61 & 321,92 & 66,32 & 322,92 \\
070_086 & 198,25 & 1462,85 & 114,91 & 198,61 & 203,43 & 1255,73 & 63,24 & 304,81 \\
070_087 & 254,05 & 2773,37 & 132,43 & 217,12 & 285,67 & 1251,04 & 100,11 & 438,19 \\
070_088 & 365,34 & 2323,62 & 126,88 & 236,75 & 243,53 & 2128,68 & 83,68 & 367,55 \\
070_089 & 309,09 & 1262,05 & 127,34 & 210,24 & 266,23 & 606,94 & 88,44 & 459,86 \\
080_085 & 375,20 & 1685,45 & 103,15 & 164,21 & 268,74 & 1486,14 & 52,50 & 257,36 \\
080_086 & 330,08 & 916,90 & 109,26 & 170,03 & 254,48 & 1968,76 & 51,57 & 261,97 \\
080_087 & 225,87 & 784,28 & 114,24 & 189,34 & 342,13 & 1492,28 & 73,57 & 317,54 \\
080_088 & 389,02 & 1035,71 & 119,92 & 214,41 & 242,63 & 1669,93 & 59,27 & 275,75 \\
080_089 & 229,66 & 1582,16 & 111,60 & 188,11 & 479,98 & 2141,83 & 66,21 & 326,12 \\
\hline
\end{tabular}

Tabela 95: Problemas-teste da classe $(10,10,15)$.

\begin{tabular}{c|cccccccc}
\hline Tempo & AT1 & AT2 & AT3 & AT4 & AT5 & AT6 & ATH1 & ATH2 \\
\hline 060_090 & 268,04 & 1116,20 & 221,19 & 332,78 & 204,99 & 361,92 & 123,66 & 546,22 \\
060_091 & 364,12 & 1177,06 & 188,50 & 333,78 & 236,59 & 533,90 & 94,71 & 418,80 \\
060_092 & 407,34 & 1266,70 & 217,05 & 352,92 & 266,42 & 1050,17 & 143,75 & 626,10 \\
060_093 & 158,12 & 1701,63 & 176,55 & 324,81 & 217,59 & 1624,49 & 84,54 & 388,16 \\
060_094 & 231,74 & 1004,93 & 204,33 & 358,41 & 273,44 & 641,07 & 112,95 & 578,95 \\
070_090 & 363,09 & 1231,44 & 176,25 & 263,75 & 215,24 & 1167,23 & 82,26 & 405,59 \\
070_091 & 238,59 & 1666,47 & 158,33 & 283,44 & 265,47 & 1870,64 & 62,43 & 322,64 \\
070_092 & 325,68 & 952,79 & 176,42 & 272,27 & 253,78 & 1000,06 & 91,06 & 420,58 \\
070_093 & 518,13 & 1639,71 & 163,19 & 244,20 & 313,05 & 1375,82 & 57,94 & 288,79 \\
070_094 & 224,53 & 532,31 & 169,92 & 251,80 & 251,55 & 1209,23 & 81,04 & 447,80 \\
080_090 & 365,28 & 1620,23 & 162,60 & 256,31 & 335,98 & 891,78 & 59,52 & 303,86 \\
080_091 & 308,31 & 2830,95 & 136,16 & 253,16 & 291,24 & 708,85 & 49,33 & 256,89 \\
080_092 & 227,93 & 1727,48 & 169,87 & 246,76 & 309,54 & 1484,83 & 67,42 & 344,73 \\
080_093 & 422,58 & 984,84 & 135,22 & 239,24 & 300,05 & 585,63 & 49,64 & 257,37 \\
080_094 & 478,56 & 845,57 & 143,96 & 250,12 & 418,99 & 884,88 & 68,88 & 424,34 \\
\hline
\end{tabular}

Tabela 96: Problemas-teste da classe $(15,15,15)$.

\begin{tabular}{c|cccccccc}
\hline Tempo & AT1 & AT2 & AT3 & AT4 & AT5 & AT6 & ATH1 & ATH2 \\
\hline 060_095 & 760,82 & 2926,22 & 421,78 & 688,60 & 598,28 & 3816,19 & 901,90 & 2534,25 \\
060_096 & 824,20 & 10878,32 & 446,38 & 647,98 & 530,12 & 1918,84 & 821,30 & 2712,67 \\
060_097 & 476,47 & 1651,47 & 428,91 & 706,47 & 492,29 & 3187,16 & 839,29 & 2843,10 \\
060_098 & 557,02 & 2268,58 & 404,84 & 674,97 & 865,99 & 1486,80 & 704,44 & 2255,23 \\
060_099 & 367,10 & 4228,83 & 460,20 & 694,14 & 473,46 & 2069,37 & 886,62 & 2479,89 \\
070_095 & 298,31 & 2574,36 & 328,60 & 539,79 & 313,97 & 3685,59 & 500,34 & 1480,82 \\
070_096 & 870,00 & 2899,47 & 330,75 & 488,48 & 786,51 & 4327,55 & 488,63 & 1348,41 \\
070_097 & 374,28 & 2809,28 & 352,67 & 537,03 & 637,14 & 2527,39 & 494,55 & 1528,81 \\
070_098 & 684,02 & 4013,47 & 344,15 & 577,69 & 942,78 & 2982,71 & 472,04 & 1443,82 \\
070_099 & 506,30 & 4142,75 & 364,04 & 547,80 & 686,70 & 4792,68 & 554,79 & 1568,53 \\
080_095 & 1111,49 & 3797,05 & 298,21 & 445,93 & 617,05 & 3953,46 & 325,93 & 1025,94 \\
080_096 & 969,51 & 4128,93 & 298,60 & 437,19 & 792,63 & 5323,41 & 316,79 & 1003,79 \\
080_097 & 1013,18 & 8291,28 & 315,43 & 491,28 & 608,54 & 3219,41 & 319,23 & 1047,47 \\
080_098 & 646,94 & 3090,47 & 307,93 & 469,58 & 1118,93 & 5877,42 & 318,62 & 1052,80 \\
080_099 & 910,44 & 3444,83 & 293,97 & 456,18 & 566,53 & 7504,46 & 359,96 & 1113,69
\end{tabular}

